\title{
A Human Tracking Mobile Robot System Based on Vision and Laser Sensor Fusion
}

by

Wen Dai

A thesis submitted to the Faculty of Graduate Studies and Research in partial fulfillment of the requirements for the degree of Master of Applied Science in Electrical Engineering

Ottawa-Carleton Institute for Electrical and Computer Engineering

Department of Systems and Computer Engineering Carleton University

Ottawa, Ontario, Canada

April, 2008

CCopyright 2008, Wen Dai 


$\begin{array}{ll}\begin{array}{l}\text { Library and } \\ \text { Archives Canada }\end{array} & \begin{array}{l}\text { Bibliothèque et } \\ \text { Archives Canada }\end{array} \\ \begin{array}{l}\text { Published Heritage } \\ \text { Branch }\end{array} & \begin{array}{l}\text { Direction du } \\ \text { Patrimoine de l'édition }\end{array} \\ \begin{array}{l}\text { 395 Wellington Street } \\ \text { Ottawa ON K1A 0N4 } \\ \text { Canada }\end{array} & \begin{array}{l}\text { 395, rue Wellington } \\ \text { Ottawa ON K1A 0N4 } \\ \text { Canada }\end{array}\end{array}$

Your file Votre référence ISBN: 978-0-494-40636-6

Our file Notre référence

ISBN: 978-0-494-40636-6

NOTICE:

The author has granted a nonexclusive license allowing Library and Archives Canada to reproduce, publish, archive, preserve, conserve, communicate to the public by telecommunication or on the Internet, loan, distribute and sell theses worldwide, for commercial or noncommercial purposes, in microform, paper, electronic and/or any other formats.

The author retains copyright ownership and moral rights in this thesis. Neither the thesis nor substantial extracts from it may be printed or otherwise reproduced without the author's permission.
AVIS:

L'auteur a accordé une licence non exclusive permettant à la Bibliothèque et Archives Canada de reproduire, publier, archiver, sauvegarder, conserver, transmettre au public par télécommunication ou par l'Internet, prêter, distribuer et vendre des thèses partout dans le monde, à des fins commerciales ou autres, sur support microforme, papier, électronique et/ou autres formats.

L'auteur conserve la propriété du droit d'auteur et des droits moraux qui protège cette thèse. $\mathrm{Ni}$ la thèse ni des extraits substantiels de celle-ci ne doivent être imprimés ou autrement reproduits sans son autorisation.
In compliance with the Canadian Privacy Act some supporting forms may have been removed from this thesis.

While these forms may be included in the document page count, their removal does not represent any loss of content from the thesis.
Conformément à la loi canadienne sur la protection de la vie privée, quelques formulaires secondaires ont été enlevés de cette thèse.

Bien que ces formulaires aient inclus dans la pagination, il n'y aura aucun contenu manquant.

\section{Canada}




\section{Abstract}

A real-time and robust human tracking robot system is presented. The robot is able to follow a walking human target based on the information obtained from a colour video camera and a laser range finder, which are mounted on the robot. For robust tracking, a model for the target person, which includes the colour and edge orientation histograms from the image data and the location of the person from the laser data, is constructed at the beginning of the tracking task. People in the field of view of the robot, including the target person, are detected by a leg detection algorithm developed in this thesis using the laser data. The target to be tracked is verified by comparing the image information with the target model. A particle filter is used to estimate the position and the velocity of the target person for guiding the robot's movements. Ego-motion compensation is embedded in the particle filtering to account for the effect of the motion of the robot itself in order to improve the tracking accuracy.

Some key requirements for robust and real-time human tracking, such as computation time, occlusion-handling, and capability of adapting to different targets are discussed. 


\section{Acknowledgements}

I would like to express my sincere gratitude to Dr. Aysegul Cuhadar and Dr. Peter X. Liu for their guidance and encouragement throughout my study.

I thank Mr. Danny Lemay, Mr. Narendra Mehta and Mr.Stephen Maclaurin for their technical support to this project.

I want to thank my family especially my husband for their endless love and support. 


\section{Table of Contents}

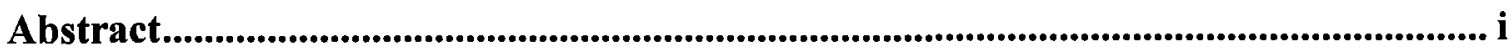

Acknowledgements .................................................................................................................. ii

Table of Contents ................................................................................................................................... iii

List of Tables ....................................................................................................................................... $\mathrm{v}$

List of Figures............................................................................................................................... vi

List of Abbreviations ................................................................................................................... viii

Chapter 1 Introduction.................................................................................................. 1

$1.1 \quad$ A Glance at Robots ................................................................................. 1

1.2 Moving Target Person Tracking …………………...................................... 2

1.2.1 The Problems for Moving Person Tracking .............................................. 2

1.2.1 The Proposed Method .......................................................................... 4

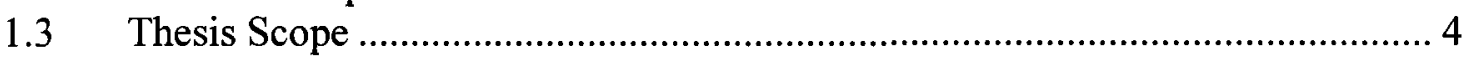

1.4 Thesis Contributions ..............................................................................

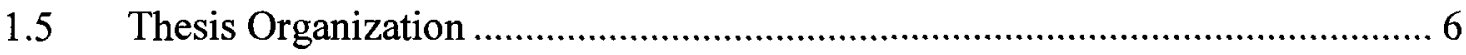

Chapter 2 Human Tracking by a Robot ...................................................................... 7

2.1 Classifications of Tracking Methods ........................................................... 7

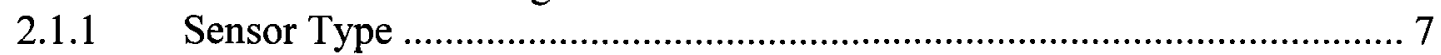

2.1.2 Fixed Platform vs. Mobile Platform ...................................................... 9

2.1.3 Tracking Algorithm .......................................................................... 10

2.2 Examples of Tracking Systems with Mobile Robots........................................ 11

Chapter 3 Proposed Framework .......................................................................... 13

3.1 Problems/Difficulties in Human Tracking...................................................... 13

3.1.1 Human Detection .......................................................................... 13

3.1.2 Target Person Recognition................................................................... 15

3.1.3 Human Tracking Control .................................................................. 16

3.2 Framework of the Human Tracking Robot System ......................................... 18

3.3 Target Person Model Construction Module........................................................ 18

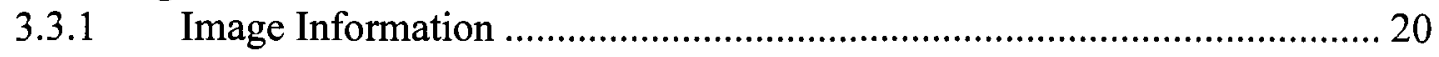

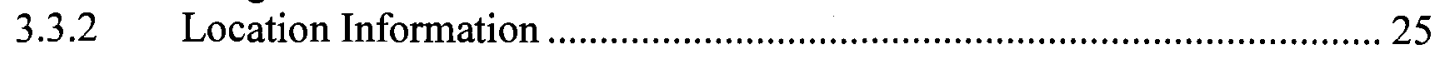

3.4. Human Candidate Detection Module............................................................. 27

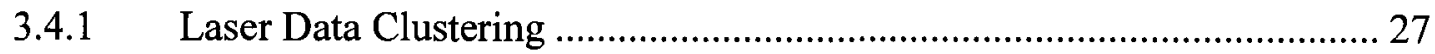

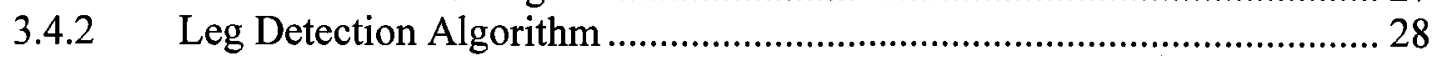

3.4.3 Leg Grouping .................................................................................... 30

3.4.4 Framework of Human Candidate Detection Module ................................. 31

3.5 Target Person Verification Module ................................................................... 31

3.5.1 Person Localization in the Image Frame ............................................ 32

3.5.2 Image Processing ………...................................................................... 40

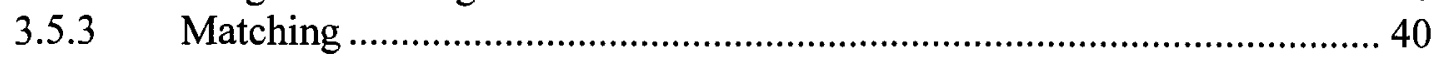




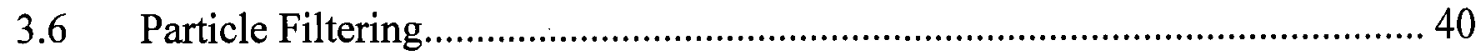

3.6.1 Basics of Particle Filters ....................................................................... 41

3.6.2 State Estimation by Particle Filtering ………………………………....... 44

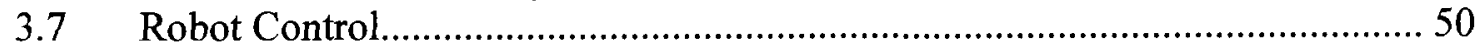

Chapter 4 System Implementation ...............................................................5 51

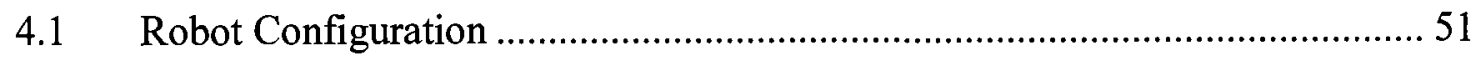

4.1.1 The Laser Range Finder .................................................................... 52

4.1.2 The PTZ Camera.................................................................................... 54

4.1.3 Program Structure .................................................................................. 54

4.2 Target Person Model Construction ............................................................... 57

4.2.1 Image Processing ................................................................................. 57

4.2.2 Location Measurement.............................................................................. 64

4.2.3 Flow Chart of the Target Person Model Construction............................... 64

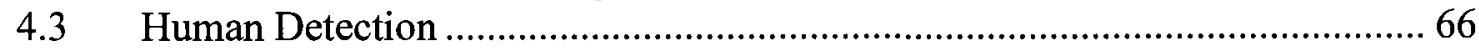

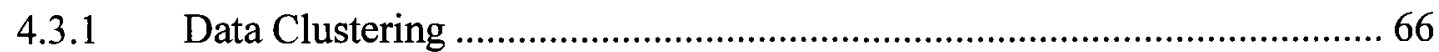

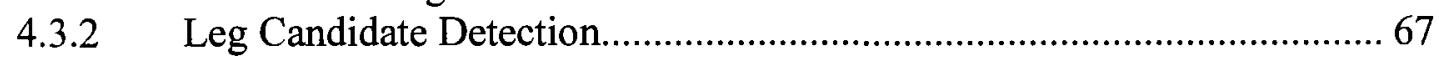

4.3.3 Human Candidate Generation................................................................. 69

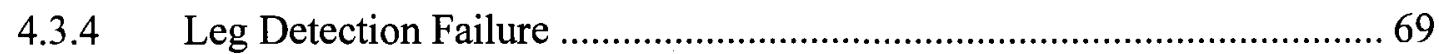

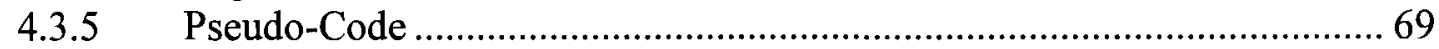

4.4 Target Person Verification......................................................................... 70

4.4.1 Rectangle Representation.................................................................... 71

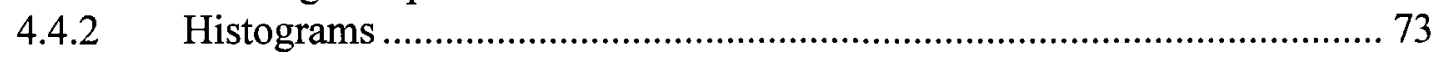

4.4.3 Image Verification Failure ........................................................................ 73

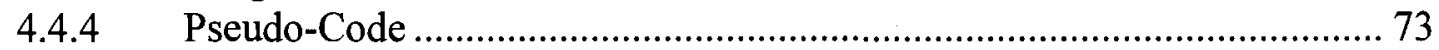

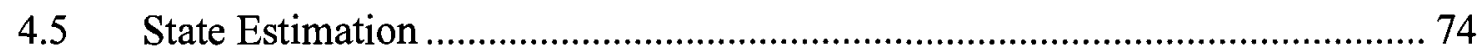

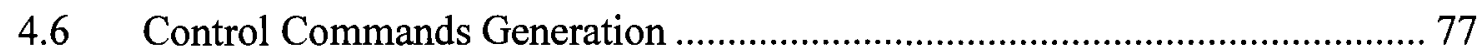

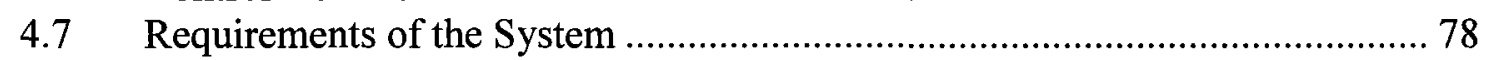

Chapter 5 Performance and Results .......................................................................... 80

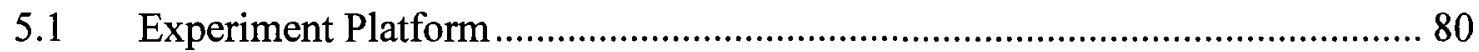

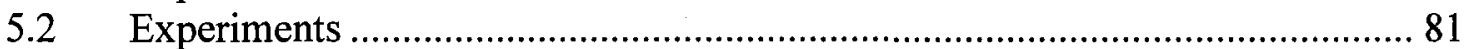

5.2.1 Comparison of Leg Detection Algorithms.............................................. 81

5.2.2 Tracking Performance of the Particle Filter......................................... 82

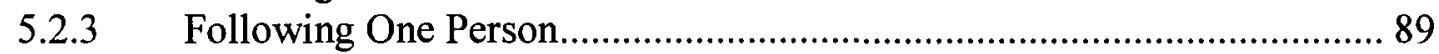

5.2.4 Target Person Recognition................................................................ 91

5.2.5 Maximum Angular Velocity ……………….......................................... 97

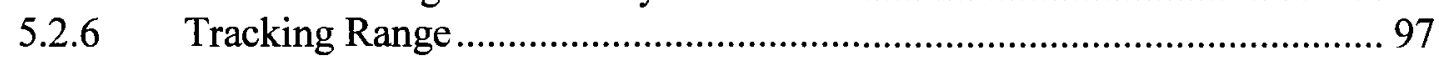

Chapter 6 Conclusions and Future Work............................................................. 98

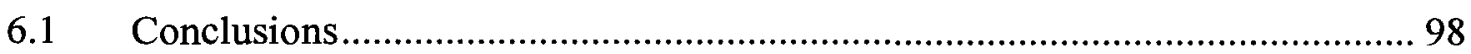

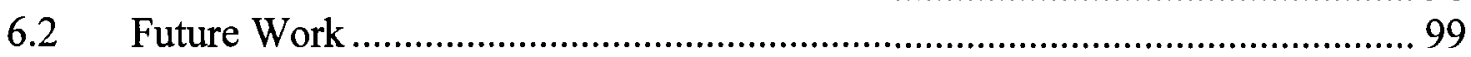

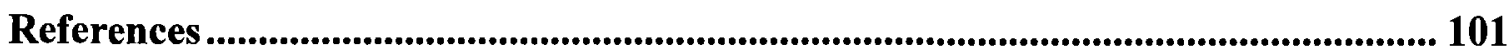




\section{List of Tables}

Table 3.1. The particle filtering algorithm .................................................................... 44

Table 4.1. Specifications of the laser range finder.......................................................52

Table 4.2. Canon VC-C4 camera specifications ............................................................. 54

Table 4.3. Relationship between the laser scan point numbers and the distance.............67

Table 4.4. The human detection algorithm ................................................................... 70

Table 4.5. Pseudo-code of the target person verification module.................................... 74

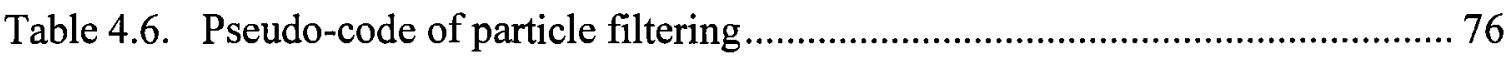

Table 5.1. The leg detection algorithms comparison...................................................... 81

Table 5.2. Average and standard deviation of the estimated velocity .............................85

Table 5.3. Average filtering time under different particle numbers ................................ 86 


\section{List of Figures}

Figure 3.1. A laser scan in the Cartesian coordinate system ........................................ 14

Figure 3.2. The framework of the human tracking system .......................................... 18

Figure 3.3. Target person model construction module …………………....................... 20

Figure 3.4. The RGB colour space and HSV colour space........................................... 22

Figure 3.5. HSV colour example: red .................................................................. 22

Figure 3.6. An example for spatial information...................................................... 23

Figure 3.7. Laser frames in the Cartesian coordinate system ........................................ 26

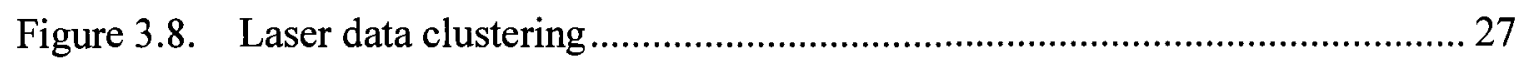

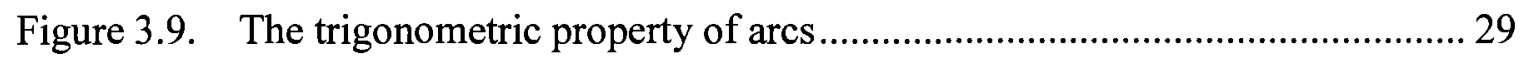

Figure 3.10. Leg patterns ......................................................................................... 30

Figure 3.11. Human candidate detection .............................................................. 31

Figure 3.12. Target person verification.................................................................... 31

Figure 3.13. Coordinate transformation.................................................................... 32

Figure 3.14. Laser frame to image frame coordinates transformation............................. 35

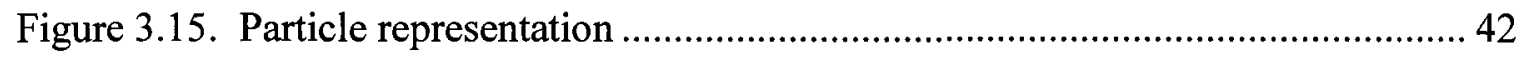

Figure 3.16. The state vector for the moving person ..................................................... 45

Figure 3.17. Ego-motion compensation.................................................................... 46

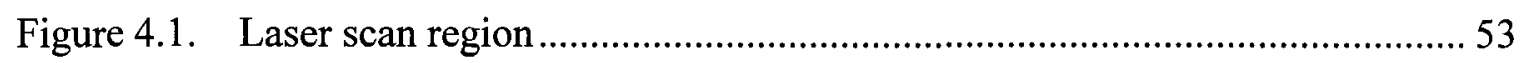

Figure 4.2. Flow chart of the human tracking robot system ........................................ 56

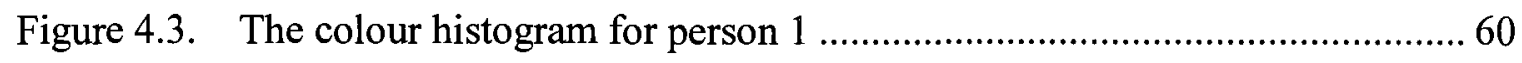

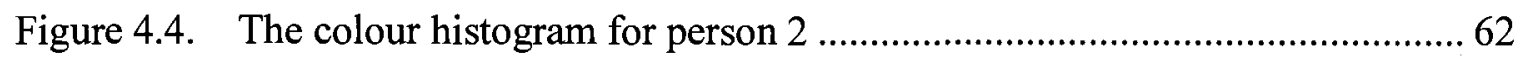

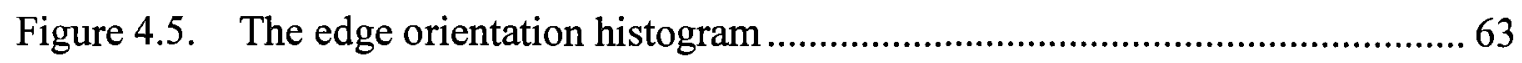

Figure 4.6. Flowchart of target person model construction ........................................... 65

Figure 4.7. A laser scan example in the polar coordinate system.................................. 66

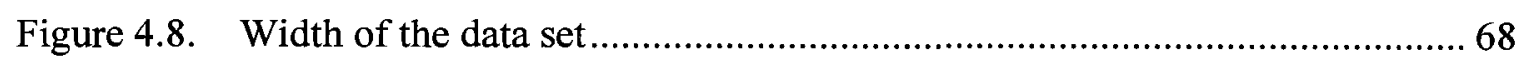

Figure 4.9. Target person verification for one-person case ....................................... 71

Figure 4.10. Target person verification for two-person case ........................................... 72

Figure 5.1. PeopleBot ${ }^{\mathrm{TM}}$ features and accessories [57] .......................................... 80 
Figure 5.2. Estimated velocities of different standard deviations $\sigma_{v_{x}}$ and $\sigma_{v_{y}} \ldots \ldots \ldots \ldots . . . . .84$

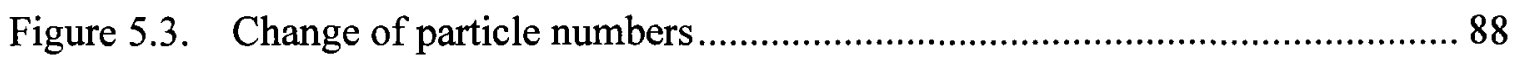

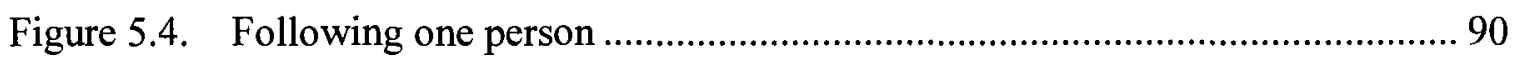

Figure 5.5. Target person recognition: person 1 ........................................................ 93

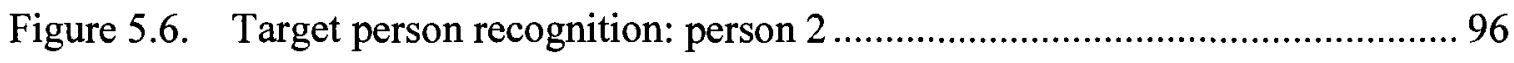




\section{List of Abbreviations}

$\begin{array}{ll}\text { ARIA } & \text { ActivMedia Robotics Interface for Application } \\ \text { EKF } & \text { Extended Kalman Filter } \\ \text { GPS } & \text { Global Positioning System } \\ \text { HRI } & \text { Human-Robot Interaction } \\ \text { HSV } & \text { Hue, Saturation and Value } \\ \text { KF } & \text { Kalman Filter } \\ \text { PF } & \text { Particle Filter } \\ \text { PTZ } & \text { Pan, Tilt, and Zoom } \\ \text { RGB } & \text { Red, Green and Blue } \\ \text { UKF } & \text { Unscented Kalman Filter }\end{array}$




\section{Chapter 1 Introduction}

In this chapter, a brief introduction to robots is presented. After that the interest is focused on human tracking robots. The problems and challenges in this field are summarized and the proposed tracking method is introduced. Then the scope, the contributions, and the organization of this thesis are described.

\subsection{A Glance at Robots}

Robots are one of the most exiting technological achievements of our time, which combine the science and engineering of mechanics, electronics, computers, and others. Industrial robots have been used in many circumstances to relieve or free people from boring and repetitive labour work. They are also used in dangerous or forbidden environments which are not suitable for human beings. Meanwhile service robots are increasingly playing an important role in our daily life, serving as assistants either in business or at home.

Many research institutes and companies producing robots are working to make robots smarter and more powerful. A possible way to achieve this goal is to equip robots with different kinds of sensors, devices and processors, such as cameras, sonar sensors, laser sensors, infrared sensors, GPS (Global Positioning System) receivers, radars, computers, etc. Some heavily visited research fields in robotics include robot localization $[1,2]$, robot mapping $[3,4,5]$, and robot navigation [6], which enable the robot work autonomously especially in unknown and even unstructured environments. Recently, Human-Robot Interaction (HRI) $[7,8,9,10]$ receives more attention which involves 
interactions between the robot and human. In the application of HRI, the robot is expected to perceive the existence of the humans and react to the instruction of the human or provide assistance/service to the human.

\subsection{Moving Target Person Tracking}

In this thesis, the interest is focused on one specific topic of HRI: real-time robotic human tracking in an indoor environment. Such a robot can be used to take care of elderly or disabled at home or provide assistance to people at work environment by keep following the people so that the robot can react to the instruction of the people promptly.

\subsubsection{The Problems for Moving Person Tracking}

The real-time requirement for human tracking robots is undoubtedly crucial for the success of tracking task. The robot should be able to keep following the target person. In other words, the robot should adapt its speed and orientation promptly according to the movement of the target person. Thus, all the information data gathered from the sensors must be processed in real-time. The processor speed and the memory size embedded with a mobile robot are usually bottlenecks due to the constraints on price, volume and weight of the robot. Making effective use of the sensor data and programming efficient code are desired.

For detecting a person from sensor data, several methods have been reported in the literature, such as face recognition $[8,9,11]$, skin segmentation $[12,32]$, silhouette matching [14], and human body detection. The first three methods extract human features, such as the human face and the skin colour from the images while the last method 
depends on the range sensors to detect the human legs $[25,27,28]$ or human torso $[15$, 16]. The vision information (image) captured from cameras can supply rich information, such as colours, edges, and contours; but it lacks distance information to locate the person in the scene, which is the key point in the human tracking application. An even bigger problem is possibly that the image processing is relatively slow and needs a great deal of computing resources, which makes it very difficult to meet the requirement for real-time applications. As a result, object tracking with image (vision) information alone is usually applied to non-real-time applications, such as some video surveillance or off-line image processing. On the other hand, range sensors such as laser range finders are powerful tools for obtaining the location of the objects with reasonably high accuracy. From the range information, one can extract the candidates of the target person by detecting the shape of the human body. However, extra information on human features is needed to differentiate the target person from all possible candidates. A possible solution is to detect the target person by his/her movement, which imposes constraints on the application that the target person must move along a restricted and relatively simple trajectory.

Another problem in the human tracking application is that the robot is also moving (that is, the human is tracked by a mobile platform). While the robot is moving, the origin of the measurement system (the robot) is changing. Therefore compensation is necessary between the previous observation and the current one. Meanwhile, the target person may change his/her pose and may be occluded by some other objects, such as a table or another person, during the course of tracking. Therefore, the tracking algorithm must have the capability of handling the shape deformation of the object, and the 
occlusion, which makes the design of the tracking algorithm more difficult than the fixed platform case.

Finally, the noise as always, from both the system and the measurement, will degrade the tracking performance if not filtered out properly.

\subsubsection{The Proposed Method}

In this thesis, the developed tracking algorithm is based on the laser data. The reason is that the laser range finder is relatively more accurate and faster to get range information than sonar sensors. We propose to fuse the range information from the laser range finder and the image information from the camera, in order to improve the tracking performance and robustness.

Specifically, a novel model of the target person, which contains both the image information of the person from the image frame and the location information of the person in the laser frame, is constructed before the robot starts to move. All the possible candidates of the target person are detected from the laser data by using the leg detection algorithm developed in this thesis. The target person is identified from other human candidates in the view of the robot by comparing it with the target model. A particle filter is employed to estimate the location and velocity of the target (walking) person combined with an ego-motion compensation procedure which is used to remove the effect of the motion of the robot itself.

\subsection{Thesis Scope}

As mentioned in section 1.1, the work in this thesis is limited to the following aspects: 
First, this thesis is focused on human tracking application in an indoor environment. The objective is to enable a mobile robot to detect a person and to keep following this particular person.

Second, only one person is marked as the target person in a tracking task. During the course of tracking, the robot is able to distinguish the target person from other people in the view field.

\subsection{Thesis Contributions}

The contributions of the thesis are listed below.

- A robust and real-time tracking method for a mobile robot to track a target person is developed. The properties of this method embody:

$\square$ Only the person who is identified by both the laser sensor and vision sensor is recognized as the target person, which minimizes the probability of false alarms compared with those algorithms using only one type of sensors.

$\square$ A particle filter is applied to estimate the location and velocity of the target person. The velocity of the moving person is assumed to be a zero-mean Gaussian distribution. With this zero-mean random-walk model, the location and velocity of the person can be estimated with higher accuracy than other motion models with rather a small number of particles. Compared with distance-based robot control methods, the robot can adapt its velocity to that of the target, so the robot can track the person more robustly. Meanwhile, the inherent odometer error of the robot is limited in one filtering cycle by embedding the ego-motion compensation in the filtering procedure. Thus, the accuracy of the state estimation is improved. 
- The robot is able to automatically create the model of the target person at the beginning when the tracking task is activated. The great benefit is that no prior database or information of the target person is required, which makes the system much easier to use and extendable to different targets. In addition, the model contains the information from both the laser and the camera, making the recognition of the target person more accurate.

- Compared with other leg-detection methods, the developed leg detection method is simple and effective. In particular, the probability of not detecting a person's legs is much lower with our method.

- The robustness and accuracy is improved by the fusion of the colour information and the edge information of the target person representation, and the fusion of the visual information and laser information for target modeling and verification.

\subsection{Thesis Organization}

This thesis is organized into six chapters. Chapter 1 gives an introduction about the research topic. Chapter 2 presents a literature review on human tracking. Chapter 3 describes the theoretic foundation and the architecture of the developed human tracking robotic system, and also the functionality of each module in the architecture is discussed in details. Chapter 4 provides the implementation details of the human tracking robot system. Chapter 5 presents the experimental results of the developed leg detection algorithm, the particle filtering module and the performance of the target personfollowing robot. Chapter 6 concludes this thesis and discusses directions for future work. 


\section{Chapter 2 Human Tracking by a Robot}

In this chapter, the state of the art in human tracking is reviewed. Several most related tracking schemes to the proposed method in this thesis are discussed in details. The drawbacks in these methods are also pointed out.

\subsection{Classifications of Tracking Methods}

A large amount of research has been done in the field of human tracking. Several characteristics distinguish one tracking method from another: single-sensor or multisensor, fixed platform or mobile platform, and the tracking algorithm.

\subsubsection{Sensor Type}

As a means of interface between the robot and the outer world, sensors play an important role in robotic applications. Typical sensors mounted on robots include vision sensors (such as cameras), range sensors (such as sonar and laser sensors), infrared sensors, microphones, etc. These sensors collect different information for the onboard processor of the robot or for the offline analysis. Infrared sensors [7] can distinguish the human from other objects based on their different temperature characteristics. Microphones are used for speech processing, for example, speech recognition or speaker recognition, which will be severely affected by the environment noise $[7,8,10]$. Vision sensors and range sensors are more widely used in human tracking applications.

Vision sensors can provide vivid and rich image information about the scene. Several kinds of vision sensors have appeared in research, such as monocular cameras, stereo cameras, and panoramic cameras. Monocular cameras is the most common type 
while stereo cameras $[17,18]$, panoramic cameras $[15,19]$ can offer wider field of view compared to monocular cameras. Furthermore, stereo cameras have the ability to provide 3D images because of the binocular property.

Given the images from the vision sensors, we segment the object of interest from the background. The object of interest can be defined manually $[13,49]$ or can be detected using the background subtraction technique $[15,20,21]$ or using motion analysis methods, for example, optical flow $[17,22,23,36]$. In most cases, the object is represented by its image features, such as colours, edges, contours, etc. Average R, G, B colours [47] or a luminance invariant version of the average colours [45] are used for object representation. Colour histogram representations are used in $[12,26,49,50]$. Colour cue is robust to the change of shape but will degrade the tracking performance when the background has the similar colour distribution with the target object. Edge or gradient cue is robust to the light variance and colour clustering. A combination of these two cues is applied to improve the tracking performance $[13,26,42,49]$.

With the image information provided by vision sensors, we can tell if there is a person in the scene and what the person's pose is. One drawback of vision sensors is they usually provide poor location information except for stereo cameras. In addition, the high computational cost of image processing tasks is a drawback for real-time applications.

Range sensors, such as sonar sensors $[24,31,47]$ and laser sensors $[24,25,27,28$, $29,33]$ are widely used sensors for the robot applications because of their ability to give the location information of the objects in the field of view of the sensor. The laser range finder has the higher accuracy and higher speed, which makes it a reliable tool to detect the object. Detecting objects with a laser range finder can be accomplished using two 
major methods. One is to find the difference between two laser frames [33, 48]. This method is feasible when the object is moving. The other is to detect the shape of the target object by analyzing the laser data. As an example, the human legs are detected based on the shape of the human legs from one laser scan in $[27,29,46]$. With the help of identifying the features of the human leg, such as the diameter of a leg and the distance between two legs, human candidates in the scene are found.

Range sensors can provide accurate location information but cannot be used for identification of objects. The target person will be easily confused with chairs or tables or other objects with leg-like features when a leg detection algorithm is applied.

Complementary-sensor applications fuse the information from different types of sensors to get more information about the object. The information from the camera and the sonar/laser range finder are fused to build the map of the environment [30] and to detect a moving person $[46,47]$.

\subsubsection{Fixed Platform vs. Mobile Platform}

The tracking procedure can be taken on a fixed platform or a mobile platform. When we talk about the fixed platform, the sensors and the platform where the sensors are mounted are fixed $[15,26]$. Generally speaking, tracking from a fixed platform is easier than tracking from a mobile platform. A typical application of fixed platform is video surveillance where the camera is fixed.

Obviously, the perceivable field of a fixed platform is limited, which restricts its application. A mobile platform is preferred in flexible tracking problems. When the platform is moving, the effect of the ego-motion of the platform itself must be accounted 
for. For example, consecutive laser frames obtained from a mobile robot are registered to extract the moving persons from the static background in [48].

\subsubsection{Tracking Algorithm}

Applying measurement results directly to the tracking system will suffer from the system noise and the measurement noise. For more accurate description about the state of the target person, a filtered state is desired. Stochastic methods such as Kalman filtering and particle filtering are applied to realize tracking procedure.

The Kalman filter (KF), including the standard Kalman filter, the extended Kalman filter (EKF), and the unscented Kalman filter (UKF), is used for motion tracking in $[34,35,39,40]$. When the posterior density of the estimated state is Gaussian and the state equation and the measurement equation are all linear, the Kalman filter is the optimal solution to the estimation. However, there are still a lot of cases that the state equation and the measurement equation are non-linear. The extended Kalman filter approximates the non-linear case with a local linearization procedure. And unscented Kalman filter has been proved to have better performance than EKF in some non-linear cases because it approximates nonlinearity better. However, both EKF and UKF are based on Gaussian posterior density assumption which is not always the case in human tracking applications.

The particle filter (PF) $[37,38,60]$ approximates the posterior density by weighted randomly sampled particles. These particles keep multiple hypotheses at each time step, which makes particle filters perform better in non-Gaussian and non-linear tracking applications. Theoretically, the more particles are drawn, the better the 
approximation will be. Thus, the main drawback of the particle filter is its relatively high computation load.

The person may move along a specific trajectory for their intentions, where Kalman filters can work well for tracking mission. However, the person may also accelerate, stop, or turn around in a sudden, which might lead the Kalman filter based filtering schemes to a failure. The particle filter based tracking schemes have proven their success in many tracking applications $[41,42,43,44,47,48]$. The details of the standard particle filters will be presented in Chapter 3.

\subsection{Examples of Tracking Systems with Mobile Robots}

A tracking system is a combination of the above three categories in section 2.1.

In [46], people are tracked with an unscented Kalman filter. People in the field of view of the robot are detected by leg detection and face detection. This scheme can fulfill a real-time people tracking procedure but a successful tracking requires that the face of the people can be detected by the camera which is not always the case. The failure of face detection will lead a failure in tracking. Also, for better tracking performance by the unscented Kalman filter, people in the scene are expected to travel with positive velocities, which is a limitation of this scheme.

A mobile robot tracks a moving object by fusing vision cue and sonar cue based on a particle filter framework in [47]. Thirty particles are used to represent different possibilities of the object's center, the object's size in the image frame and the distance from the robot measured by the sonar sensor. Average RGB colours are used to represent the object. Applying the particle filter to the image frames challenges the computation capability of the onboard computer. In addition, the state estimation with a relatively 
small number of particles would lead to a less accurate tracking performance and even a loss of tracking. Furthermore the average colour object representation in RGB plane is prone to be interfered by luminance variation.

A laser based leg detection and particle filter are implemented for peoplefollowing application [41]. Without any identification information, e.g. information from vision sensors, the differentiation between the target person and other objects with similar shape is impossible. This drawback also exists in other human tracking applications which work on laser data only. 


\section{Chapter 3 Proposed Framework}

In this chapter, the problems and difficulties for human tracking using a mobile robot are explained. The framework of the developed human tracking robot system is presented. Each module of this framework is discussed in details.

\subsection{Problems/Difficulties in Human Tracking}

The necessary functions of a robust human tracking mobile robot system are defined as: the robot should be able to detect the walking target person in its field of view and keep following him/her robustly even under occasional occlusion. The occlusion can be caused by the person himself/herself, for example changing poses, or caused by other objects. The issues and challenges to realize these functions for a human tracking system are:

1. To detect the persons in the field of view of the robot.

2. To distinguish the target person from the other persons or objects in the view.

3. To control the robot to follow the target person at an appropriate velocity.

\subsubsection{Human Detection}

The sensors on the robot provide the original data for further processing in robotic applications. For detecting the human in the scene, first we need to decide which sensors are most helpful for the purpose of human tracking. The possible sensors that can be used in this thesis project are a laser range finder, two sonar rings and a PTZ (pan, tilt, and zoom) colour video camera. 
The Laser range finder and the sonar arrays are both range sensors whereas laser is more accurate and faster than sonar. Therefore the laser sensor is preferred to the sonar sensor for human detection. The laser range finder is mounted on the robot at about $40 \mathrm{~cm}$ above the ground. Such a mounting height is good for the laser range finder to detect the human's legs. The laser range finder sends a narrow laser beam towards the object and the laser beam will be reflected by the closest object on the way and received by the laser range finder. Then the distance and the angle between the center of the laser range finder and the reflection point are measured. After one laser scan, the resulting laser frame, which is composed of the distance and angle data of each reflection point, is a point to point mapping: one angle datum is corresponding to one distance datum. An example of a laser scan, or a laser frame, in the Cartesian coordinate system is shown in Figure 3.1. The dots in this figure denote objects. One drawback of the laser range finder is that it cannot identify a human object by itself. All human leg-like objects will be recognized as human legs by leg detection algorithms.

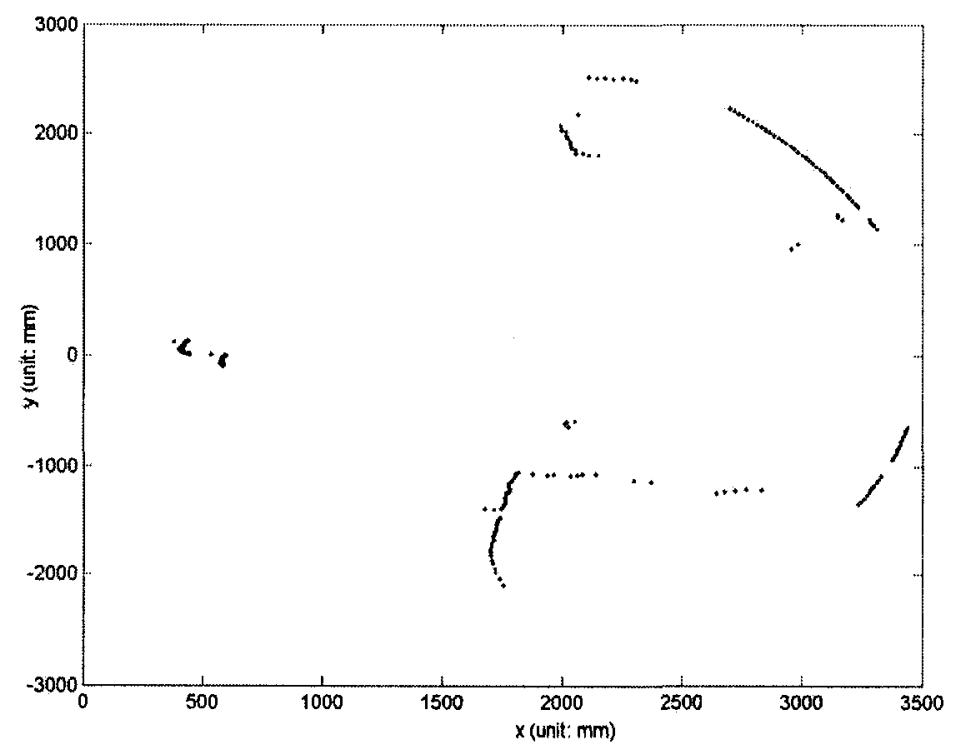

Figure 3.1. A laser scan in the Cartesian coordinate system 
The PTZ colour video camera is about 1 meter high above the ground, which can catch the image information of the human body. The width and the height of the image are 320 pixels and 240 pixels respectively.

It is noted that since a laser frame contains much less information than an image frame, the time and computation power needed for processing laser data are much less than image processing tasks. Due to the requirements for real-time and constraints on the available robot computation power, a leg detection algorithm is developed based on the laser data. All the leg-like objects will be detected using this leg detection algorithm. The detected "legs" are referred to as leg candidates in this thesis. Further, all the "legs" are assigned to hypothesis "persons". The quotation marks here mean that not all hypothesis persons are real persons. They may be a table or a desk. These hypothesis "persons" are referred to as human candidates.

\subsubsection{Target Person Recognition}

Tables, chairs, persons, or other objects may be detected as human candidates by the leg detection algorithm. The objective of person recognition is to differentiate and identify the target person from all these human candidates. As mentioned before, this cannot be fulfilled by laser data processing. However, the target person can be identified in the image frame by comparing the information of the target person with that of the human candidates. The most similar candidate will be regarded as the target person. Therefore, a pre-defined model of the target person is needed.

The target person model needs to be constructed at the beginning of the tracking process. For modeling the target person, two types of data sources are available. One is the laser frames from the laser range finder, and the other is the image frames from the 
camera. These two data sources have correspondence to each other: when the person is moving farther away from or nearer to the robot (the distance is derived from the laser frame), the size and the position of the person in the image frame will change accordingly. The target person model is composed of:

1. The location of the target person in the laser frame the time the person starts moving. This location will determine the position and the size of the person in the image frame.

2. The normalized image information, such as the colours and edges, of the person. For distinguishing the target person from other human candidates, the image information of the target person will be used to compare with the image information of each human candidate. So, the image information of the target model needs to be normalized in order to be independent of the image size of the person.

If a human candidate is the target person, according to the relationship between the location in the laser frame and the size and position in the image frame, the normalized image information of this human candidate should be equal or sufficiently close to the target model. A measure of similarity is employed to evaluate the distance. The human candidate, which is the most similar to the target model, will be marked as the target person.

\subsubsection{Human Tracking Control}

A human tracking mobile robot should be able to control its own movement in order to keep following the target: when the person is turning, the robot turns as well; when the person is walking faster or slower, the robot should adjust its velocity 
accordingly. A straightforward method to control the robot is to generate turning commands for the robot based on the angle of the target person in the laser frame, and to set the translation velocity of the robot based on the velocity of the target person. However, there are three challenges:

1. The robot should react to the movement of the person promptly.

2. The localization of the person is obtained by the laser-data based human detection. The measurement noise is unavoidable. Two noise sources are included, one is the noise of the laser range finder itself, and the other is the leg detection process (the position of the human leg contains errors).

3. The velocity of the person is a hidden parameter that should be found out.

In order to deal with the above challenges, a Bayesian filter is used to estimate the state of the person, such as the location and velocity of the person, to achieve a smooth tracking from the noisy measurement.

The target person may change his or her velocity or direction in a sudden or unpredictable manner. The particle filtering is superior to the traditional Kalman filtering for the non-linear/non-Gaussian situations. Also note that the robot is moving when following the moving person, thus the motion of the robot itself must be compensated for the correct estimation of the person's state.

Based on the location and velocity of the person estimated by the particle filter, commands are sent to the robot controller that performs the low-level control of the robot movement. 


\subsection{Framework of the Human Tracking Robot System}

Based on the discussion from sections 3.1.1 to 3.1.3, the framework of the human tracking robot system is derived as shown in Figure 3.2. This system contains five modules, which work in two consecutive stages: the pre-tracking stage and the tracking stage. The two stages are separated by the dashed line in Figure 3.2. The target person model construction module builds the model of the moving target person in the pretracking stage when the robot is still. The human candidate detection module, the target person verification module, the particle filtering module, and the robot control module work together to fulfill person-following task in the tracking stage. The involved sensor(s) in each module are included in the parentheses in Fig 3.2. Each module will be discussed in details from section 3.3 to section 3.7 .

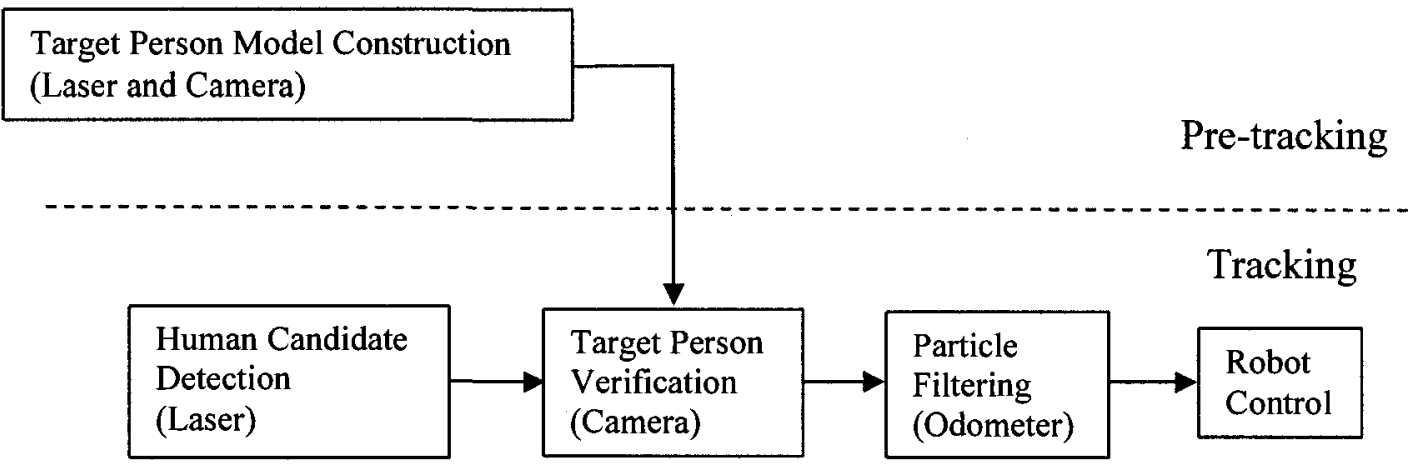

Figure 3.2. The framework of the human tracking system

\subsection{Target Person Model Construction Module}

In the human tracking application, the robot reacts to the movement of the target person in its field of view, so it is reasonable that the construction of the target person model is activated by the movement of the person. 
There are quite a few methods for segmenting a moving person in an image from the background, such as background subtraction and optical flow. A simple method for detecting the moving person is to get the difference frame. If the target person is the only moving object in the field of view of the camera, the difference frame of two successive image frames, which is obtained by subtracting the current frame from the previous frame, will locate the person with the image coordinates and give the size of the moving person in the image frame. A laser frame can be viewed as an image with less data compared with the image frame. The difference frame of two laser frames locates the person with respect to the world coordinates originated at the laser range finder. These two locations have one-to-one correspondence: when the person is at $\left(x_{l}, y_{l}\right)$ in the laser frame, the region of the person in the image frame will have the center at coordinate $\left(x_{i}, y_{i}\right)$ with size $A$.

The image information of the target person is extracted from the image frame. For eliminating the sensor noise, a smooth step can be applied to the two consecutive image frames before the frame differencing. A threshold is applied to the difference image to remove the pixels with slighter brightness changes. The thresholded difference image gives the outline of the motion region. A bounding rectangle is generated by the outline. This rectangle is used to segment the target person from the background in the colour image frame. Considering the advantages and disadvantages of the colour cue and edge cue, the combination of these two is employed to represent the target.

The location of the target person in the laser frame is obtained from the laser difference frame. To remove the difference laser readings that do not belong to the 
moving person, a leg detection algorithm can be used to help locate the legs in the difference laser frame.

The target person model, which consists of the location of the person in the laser frame, the size, the colour and edge information of the rectangle person image, is stored for the target person verification module. The functional structure of the target person model construction module is shown in Figure 3.3.

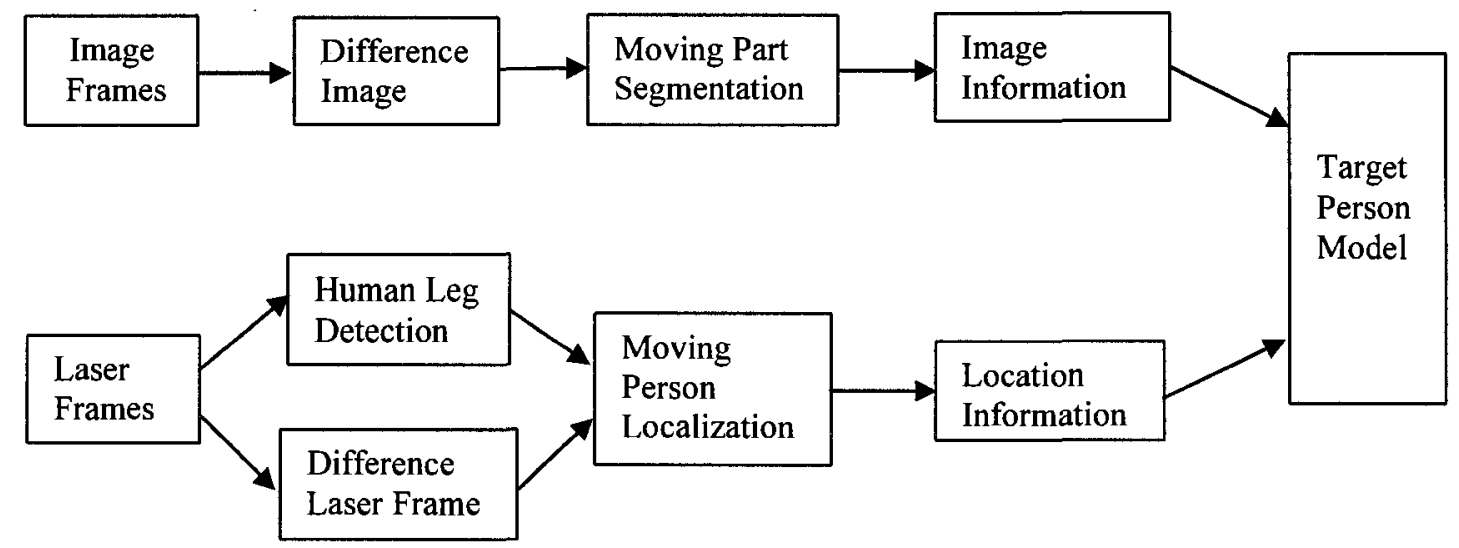

Figure 3.3. Target person model construction module

\subsubsection{Image Information}

The colour histogram and edge orientation histogram of the segmented rectangle image are calculated to represent the target person and are normalized to 1 in order to be independent of the rectangle size.

\subsubsection{Colour Histogram}

The most widely used colour space for computer display is RGB (Red, Green, and Blue). The luminance of each pixel in a RGB image is embedded in the $R, G$, and $B$ channels. So when the robot is moving, the traditional RGB colour representation will suffer from the luminance variance (colours will change under different luminance 
conditions). To obtain colour features that are invariant to the luminance, the HSV (Hue, Saturation, and Value) colour space is used. Hue represents the colour, such as red, yellow, green, etc. Saturation represents the colour strength, which determines whether the colour is pastel or sharp. And Value determines how bright or dark the colour is. Therefore, in the HSV colour space, the luminance is represented by a separate channel: the Value channel. Different from the RGB colour space, the HSV colour space is a nonlinear colour space. The transformation between the RGB colour space and the HSV colour space is defined as:

$$
\begin{aligned}
& V=\max (R, G, B) \\
& l=\min (R, G, B) \\
& S=\left\{\begin{array}{cc}
0, & V=0 \\
\frac{V-l}{V}, & \text { otherwise }
\end{array}\right. \\
& H=\left\{\begin{array}{cc}
0, & V=l \\
\frac{60(G-B)}{V-l}, & V=R \\
\frac{60(B-R)}{V-l}+120, & V=G \\
\frac{60(R-G)}{V-l}+240, & V=B \\
H+360, & H<0
\end{array}\right.
\end{aligned}
$$

where $R, G$, and $B$ are values of the three channels of a pixel in the RGB colour space. The range of each channel is $[0,1] . H, S$ and $V$ are the value of the three channels of a pixel in the HSV colour space. After the transformation, $H$ varies in $[0,360)$, and $S$ and $V$ lie in range $[0,1]$.

The RGB colour space is cubic (Figure 3.4 (a)). According to Equation (3-1), the HSV colour space is cylindrical. However, in practice the Saturation levels and Hues 
become more difficult to distinguish as the Value approaches 0 . Thus a conical representation is more popular (Figure 3.4 (b)).

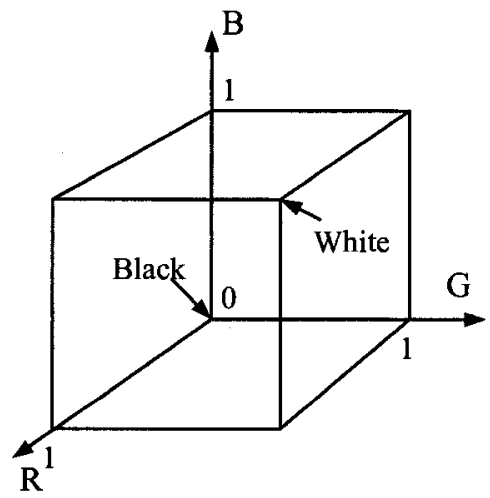

(a) RGB colour space

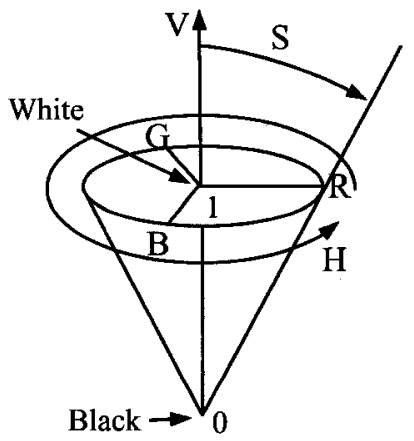

(b) HSV colour space

Figure 3.4. The RGB colour space and HSV colour space

A colour histogram is useful to describe the colour distribution. It can be a 1D (the histogram for one channel), 2D or 3D histogram depending on specific applications. A modified 2D HS histogram introduced in [50] is employed in this thesis. Before building the colour histogram, let us take a look at the colours in the HSV colour space.

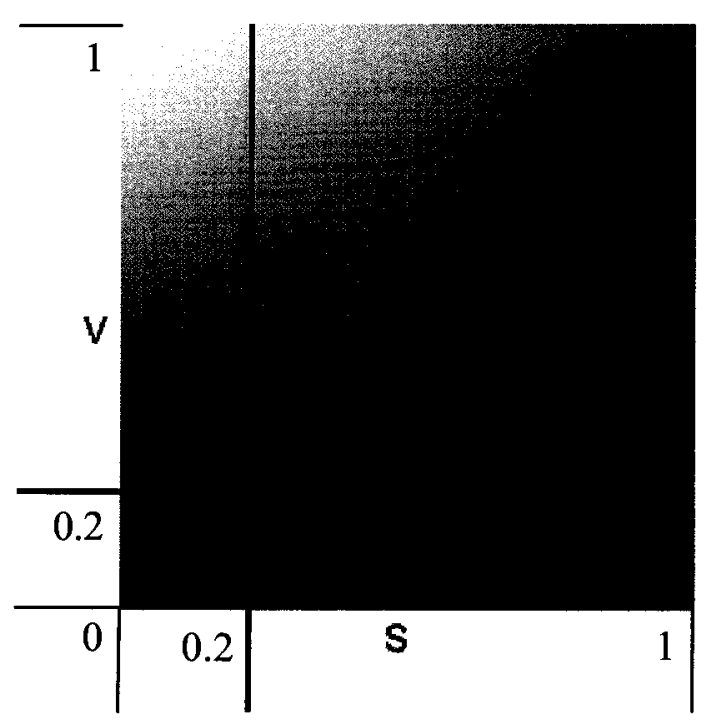

Figure 3.5. HSV colour example: red 
Figure 3.5 shows how the red looks like in the HSV colour space. From this figure, it can be found that when $V<0.2$, the red colour is close to black and when $S<0.2$, the red colour is close to gray. That is to say $H$ will not be a perceivable colour if $V<0.2$ or $S<0.2$. Based on this observation, the method to build the modified 2D HS colour histogram is composed of the following four steps.

1. Segment the range of $H, S$, and $V$ into several equal bins with numbers as $N H, N S$ and $N V$ respectively. The bins for $H$ and $S$ components are referred to as colour bins and the bins for $V$ component are referred to as brightness bins. The total number of bins of the colour histogram is $N H \times N S+N V$.

2. Pixels with $V<0.2$ or $S<0.2$ are assigned to $N V$ brightness bins.

3. Pixels with $V$ and $S$ above the two thresholds are assigned to $N H \times N S$ colour bins.

4. Normalize the colour histogram in order to be independent of the pixel numbers.

A significant drawback of the colour histogram is that it lacks spatial information. For example, Figure 3.6 (a) and Figure 3.6 (b) are two rectangles with identical size. Each rectangle has two equal size sub-rectangles: one white and one black. Obviously, the rectangles in Figure 3.6 (a) and Figure 3.6 (b) have opposite spatial colour distributions. The colour histograms for these two rectangles will be the same whereas in fact they represent different objects.

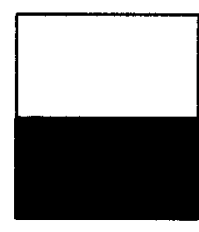

(a)

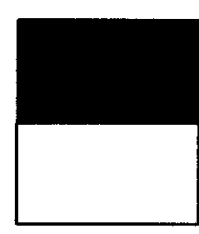

(b)

Figure 3.6. An example for spatial information 
For integrating the spatial information into the colour histogram, the segmented rectangle image, which represents the person, is split horizontally into two parts: the upper part and the lower part. Each part builds its own $N H \times N S+N V$ bins $2 \mathrm{D}$ colour histogram, which is called the sub-histogram. The total bins of the colour histogram for the segmented rectangle image therefore are $2 \times(N H \times N S+N V)$. The two subhistograms are renormalized together by multiplying 0.5 to form the whole colour histogram.

\subsubsection{Edge Orientation Histogram}

When the colour distribution of the background is similar with the target person, the similarity measure based on the colour histogram faces the challenge to distinguish the target person from the background. In this case, the edge cue is a useful compensation for the colour cue. The edge orientation histogram was first introduced for gesture recognition [51] and has been used for video tracking in [13, 49]. It is constructed for the whole segmented person image and the steps involved are:

1. Compute the edge image by applying an edge detector to the intensity image.

2. Ccompute the strength and orientation of each pixel in the edge image.

3. Segment the orientation range into equal bins.

4. Set a threshold for the strength. The pixels with strength larger than the threshold are assigned to associated orientation bins.

5. Normalize the edge orientation histogram to 1 to be independent to the image size. 
The Sobel edge detector is chosen to compute the edge images because of its simplicity and computational efficiency. The vertical and horizontal Sobel operators $S_{x}$ and $S_{y}$ are:

$$
S_{x}=\left[\begin{array}{lll}
-1 & 0 & 1 \\
-2 & 0 & 2 \\
-1 & 0 & 1
\end{array}\right] \text { and } S_{y}=\left[\begin{array}{ccc}
-1 & -2 & -1 \\
0 & 0 & 0 \\
1 & 2 & 1
\end{array}\right]
$$

The vertical and horizontal edge images $E_{x}(x, y)$ and $E_{y}(x, y)$ are computed as

$$
\left\{\begin{array}{l}
E_{x}(x, y)=S_{x}(x, y) \otimes I(x, y) \\
E_{y}(x, y)=S_{y}(x, y) \otimes I(x, y)
\end{array}\right.
$$

where $I(x, y)$ stands for the intensity image, and sign $\otimes$ stands for the $2 \mathrm{D}$ convolution operation. The magnitude $M(x, y)$ image and the orientation $\beta(x, y)$ image are calculated by

$$
\begin{gathered}
M(x, y)=\sqrt{E_{x}(x, y)^{2}+E_{y}(x, y)^{2}} \\
\beta(x, y)=\arctan \left(E_{y}(x, y) / E_{x}(x, y)\right)
\end{gathered}
$$

\subsubsection{Location Information}

The location of the target person is primarily derived from the difference laser frame. Figure 3.7 gives an example of the previous laser frame (Figure 3.7 (a)), the current laser frame (Figure 3.7 (b)) and the difference laser frame (Figure 3.7 (c)) in the Cartesian coordinate system. The robot (or the laser range finder) is located at $(0,0)$. A person is walking in front of the robot in this example. The locations of the person in the previous and current laser frames are highlighted by an ellipse in Figure 3.7 (a) and Figure 3.7 (b). We are unable to determine which point corresponds to the target from the 
difference laser frame (Figure 3.7 (c)) if no prior information about the location of the person is available. The person may be at the position that is marked as an ellipse or at the position that is marked as a rectangle in Figure 3.7 (c). In order to distinguish the person from the background in Figure 3.7 (c), a leg detection algorithm is applied to the previous laser frame to generate several leg candidates. If one leg candidate in the previous laser frame contains the difference points in the difference laser frame, the location of this leg candidate is regarded as the location of the moving person. The leg detection algorithm will be discussed in details in section 3.4.

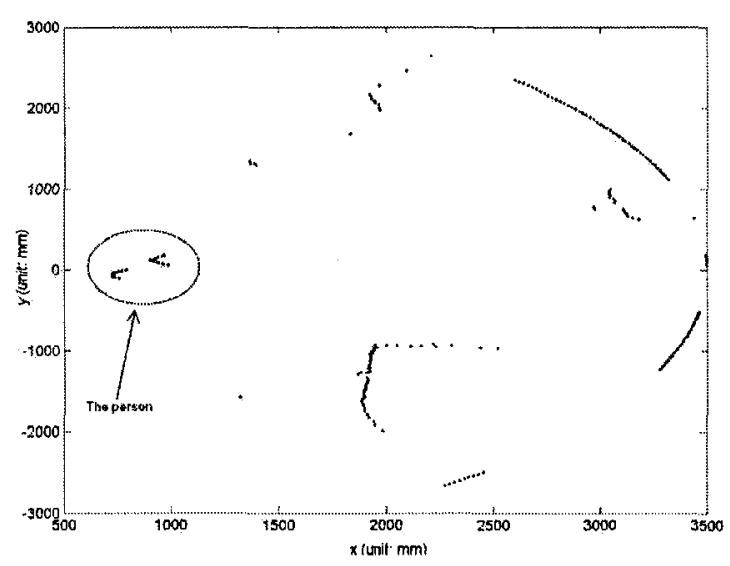

(a) The previous laser frame

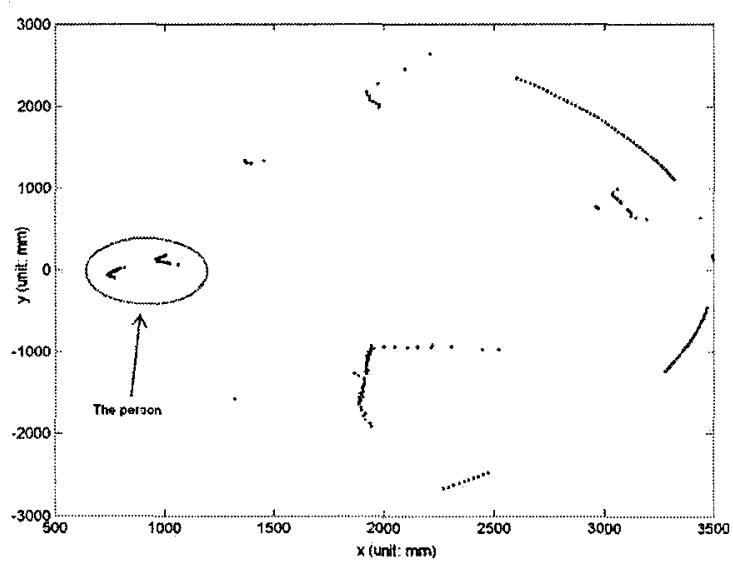

(b) The current laser frame

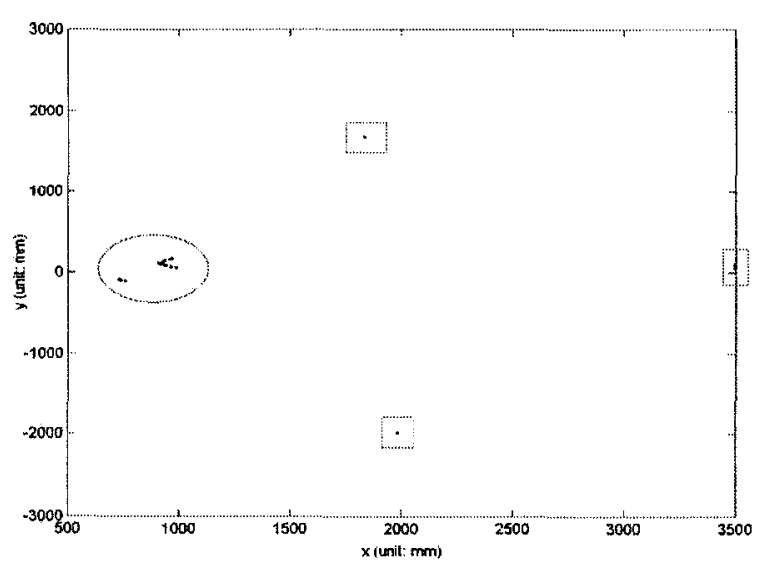

(c) The difference laser frame

Figure 3.7. Laser frames in the Cartesian coordinate system 


\subsection{Human Candidate Detection Module}

The human candidate detection module is used to find out how many persons are in the view field of the robot and what the location of each person is, which is mainly based on the laser data.

\subsubsection{Laser Data Clustering}

To detect human legs from a laser frame, the first step is to perform data clustering. Two adjacent laser readings are assigned to be in the same data cluster if they have a relatively small difference between each other. The clusters with too few or too many laser readings, which are unlikely to be a human leg, are discarded. Taking Figure 3.8 as an example, the data clustering is performed by the following criterion

$$
\left|p_{k}-p_{k-1}\right|<\varepsilon
$$

where $p_{k-1}$ and $p_{k}$ are two adjacent laser readings that represent the distance between the objects and the laser range finder, and $\varepsilon$ is a small constant.

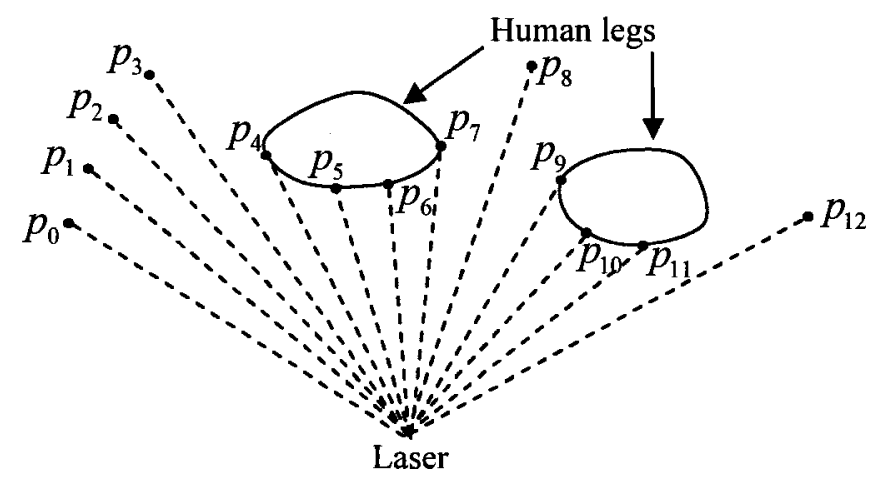

Figure 3.8. Laser data clustering

In Figure 3.8, after data clustering, the laser readings $\left(p_{0} \sim p_{12}\right)$ are divided into five clusters, which will be referred to as sets $\mathrm{S}_{0}=\left\{p_{0}, p_{1}, p_{2}, p_{3}\right\}, \mathrm{S}_{1}=\left\{p_{4}, p_{5}, p_{6}, p_{7}\right\}$ 
and $\mathrm{S}_{2}=\left\{p_{8}\right\}, \mathrm{S}_{3}=\left\{p_{9}, p_{10}, p_{11}\right\}$, and $\mathrm{S}_{4}=\left\{p_{12}\right\}$ in the following sections. The two sets $\mathrm{S}_{2}$ and $\mathrm{S}_{4}$ will be discarded because they both have only one laser reading.

\subsubsection{Leg Detection Algorithm}

In the tracking stage, the target person should be recognized even he or she does not move. Human leg detection algorithms have been developed for detecting people standing still in $[29,46]$. One of the methods is based on the local minimum detection [46]. After data clustering, the sets that are closest to the robot and have reasonable width and reasonable distance to other sets are recognized as legs. This method can easily mistake for other objects as human legs. It is suitable in spacious environment.

Another popular method is to explore the shape of a human leg [29]. The original shape of a human leg can be approximated as a cylinder. Theoretically, an arc region with reasonable width in the laser frame can be found if the leg is not occluded by another leg or other objects. In [29], the human leg is detected by finding a circle in the laser frame. It is based on the trigonometric property of arcs. That is, each point in an arc has congruent angles in respect to the extremes. For example, in Figure 3.9, $P_{A}$ and $P_{B}$ are the two extremes of the arc, $O_{c}$ is the center of the circle, and $P_{C}$ and $P_{D}$ are two points in the arc. The inscribed angles $\angle P_{A} P_{C} P_{B}$ and $\angle P_{A} P_{D} P_{B}$ are equal.

$$
\angle P_{A} P_{C} P_{B}=\angle P_{A} P_{D} P_{B}
$$




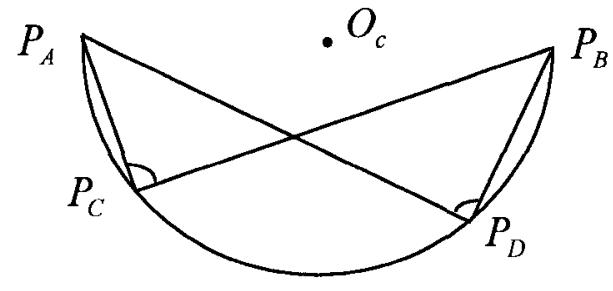

Figure 3.9. The trigonometric property of arcs

The inscribed angle on the arc should be around $90^{\circ}$ if an ideal human leg, which is a semicircle, is detected. In practice, the arc assumption is too strong to be satisfied because of the different materials of the pants and different poses of the walking person, especially when a person is wearing pants made of hard materials, like jeans. This method gives low false alarm probability but high undetected probability.

In the human tracking application, an undetected error is more serious than a false alarm. Because the false alarms can be reduced in the target person verification step whereas a miss may lead to a fail in the tracking system. Furthermore, the original leg position has been obtained when the target model is constructed. Consequently, the single leg detection criteria for the human tracking robot are developed as follows:

1. The closest point to the robot in the set (the set after data clustering) should not be the ends of the set. Specifically, for a set $S=\left\{p_{k}, p_{k+1}, \ldots, p_{k+m}\right\}$, it should satisfy

$$
\left\{\begin{array}{l}
p_{\min } \neq p_{k} \\
p_{\min } \neq p_{k+m}
\end{array}\right.
$$

where $p_{\min }$ is the closest laser point to the robot in this set. Still taking Figure 3.8 as an example, the closest point to the robot in set $S_{0}$ should not be $p_{0}$ or $p_{3}$. 
2. The leg width should be within an appropriate range. The usual diameter of a human leg is around $0.1 \mathrm{~m}$ to $0.25 \mathrm{~m}$. So the set that the distance between two ends of a set is far from this range is less possible to be related to a human leg.

3. The position of the detected leg should be close to the position of the leg(s) in the previous time step. The person is moving with a walking speed. The distance the person travels between two observations can not be too long.

The sets can be regarded as leg candidates if they satisfy the above single leg detection criteria. With these criteria, the chance that real human legs are rejected is very low. It should be noted that in the target model construction stage, only criterion 1 and 2 are used to detect the human legs.

\subsubsection{Leg Grouping}

The purpose of leg grouping is to assign the leg candidates to persons.

Given the leg candidates, two leg patterns are considered: two-leg, Figure 3.10 (a) and single-leg, Figure 3.10 (b). Figure 3.10 (c) is a special case, in which one leg is partly occluded by the other. If there is an apparent gap between these two legs, it will be covered by the two-leg pattern. Otherwise it will be covered by the single-leg pattern.

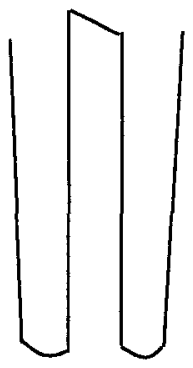

(a) Two-leg

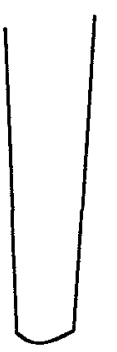

(b) Single-leg

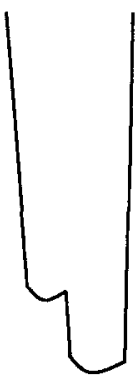

(c) Special case

Figure 3.10. Leg patterns 
Two leg candidates with reasonable distance are regarded to belong to one person.

The rest of the leg candidates that do not have such relationship will generate persons by themselves. After leg grouping, the human candidates are generated.

\subsubsection{Framework of Human Candidate Detection Module}

Based on the discussion from section 3.4.1 to section 3.4.3, the framework of the human candidate detection module is built in Figure 3.11.

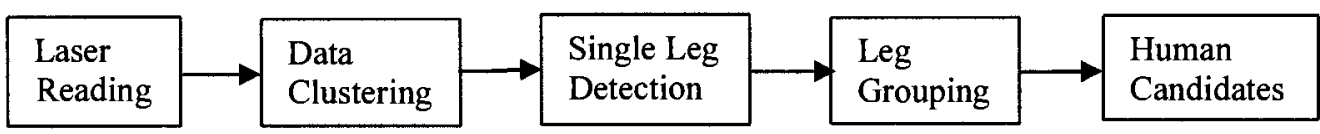

Figure 3.11. Human candidate detection

\subsection{Target Person Verification Module}

The target person verification module (Figure 3.12) chooses the most likely target person from all the possible human candidates by comparing the image information of each human candidate with the target model. A key point here is to find the corresponding image region of each human candidate in the image frame based on the location information of the human candidate in the laser frame.

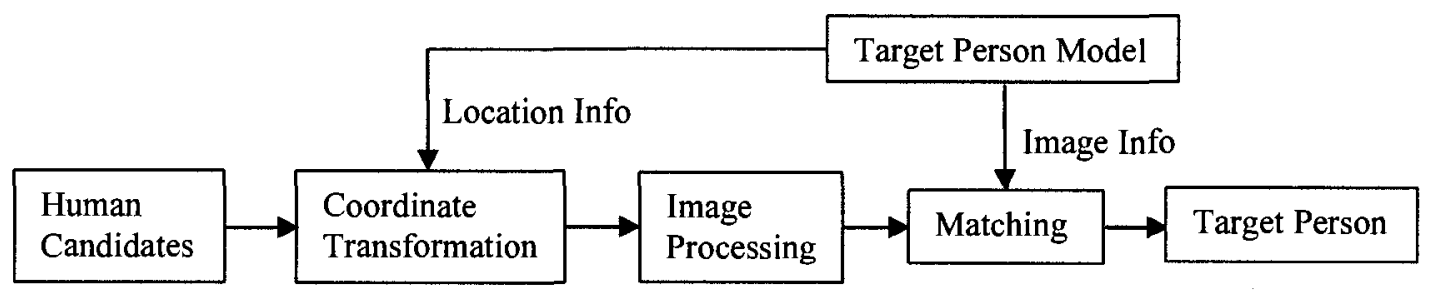

Figure 3.12. Target person verification 


\subsubsection{Person Localization in the Image Frame}

Notice that the target person in the image frame is bounded with a rectangle in the target person model construction stage and the target person model contains the colour and edge information of the rectangle image. The human candidates in the current image frame will also be bounded by rectangles. The center and the size of each human candidate rectangle in the current image frame are obtained by the coordinate transformation from the laser frame to the image frame.

\subsubsection{World to Image Coordinate Transformation}

The world plane is the plane that is in front of the camera and perpendicular to the level ground. The image plane is the plane where the film of the camera is. The object in the world plane will be projected to the image plane through the camera lens if this object is within the field of view of the camera. The relationship between the coordinates in the world and image planes is described in Figure 3.13.

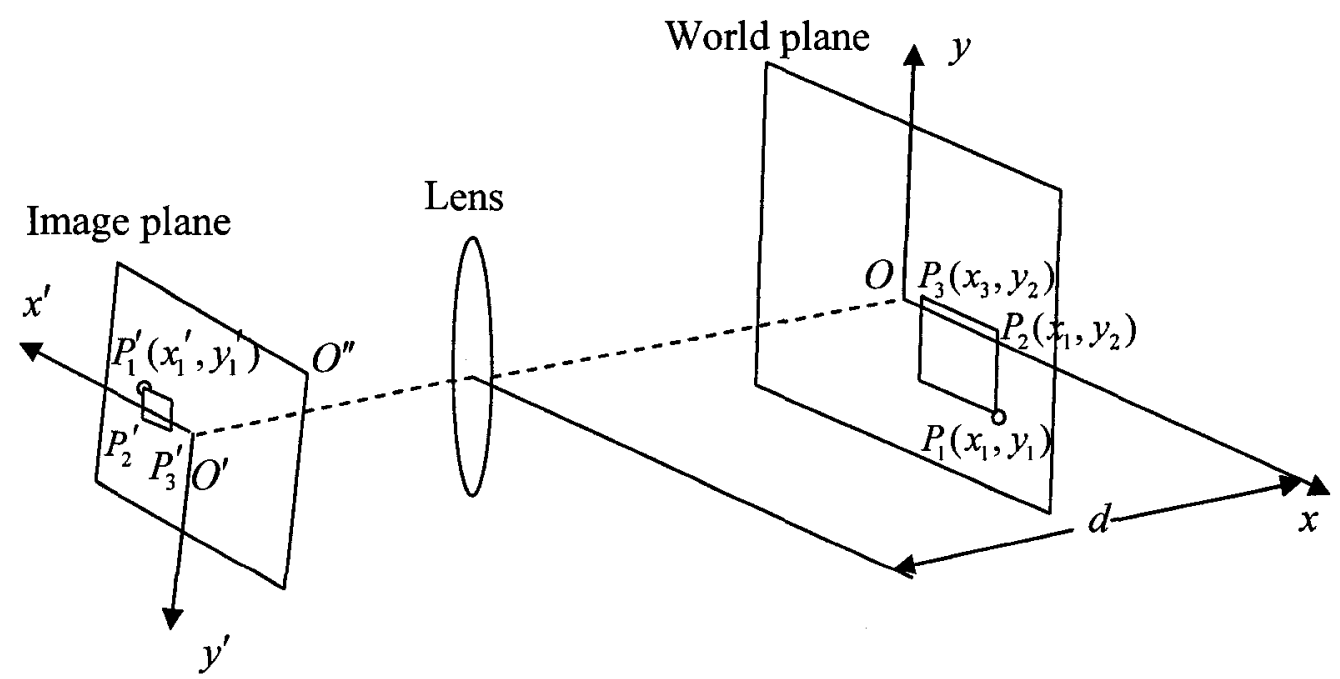

Figure 3.13. Coordinate transformation 
In Figure 3.13, $O$ and $O^{\prime}$ are the centers of the world plane and the image plane respectively, the center of the camera lens is on the line $O O^{\prime}$, and $d$ stands for the distance between the world plane and the camera lens. For the point $P_{1}\left(x_{1}, y_{1}\right)$ in the world plane, the coordinate of its corresponding point $P_{1}^{\prime}\left(x_{1}^{\prime}, y_{1}^{\prime}\right)$ in the image plane is calculated by [54]

$$
\begin{aligned}
& x_{1}^{\prime}=k f \frac{x_{1}}{d} \\
& y_{1}^{\prime}=l f \frac{y_{1}}{d}
\end{aligned}
$$

where $k$ and $l$ are the horizontal and vertical scale factors of the camera lens respectively, and $f$ is the focal length of the camera.

Again in Figure 3.13, there is a rectangle region in the world plane. Three vertexes of the rectangle are denoted as $P_{1}\left(x_{1}, y_{1}\right), P_{2}\left(x_{1}, y_{2}\right)$ and $P_{3}\left(x_{3}, y_{2}\right)$. Their corresponding vertexes in the image plane are denoted as $P_{1}^{\prime}\left(x_{1}^{\prime}, y_{1}^{\prime}\right), P_{2}^{\prime}\left(x_{1}^{\prime}, y_{2}^{\prime}\right)$ and $P_{3}^{\prime}\left(x_{3}^{\prime}, y_{2}^{\prime}\right)$ (the coordinates of $P_{2}^{\prime}$ and $P_{3}^{\prime}$ are omitted in Figure 3.13). The width of the rectangle in the world plane is the line segment $P_{3} P_{2}$ and the height is the line segment $P_{2} P_{1}$. The width of the rectangle in the image plane is the line segment $P_{3}^{\prime} P_{2}^{\prime}$ and the height is the line segment $P_{2}^{\prime} P_{1}^{\prime}$. The length of the line segments $P_{3}^{\prime} P_{2}^{\prime}$ and $P_{2}^{\prime} P_{1}^{\prime}$ is calculated below based on Equation (3-9) and Equation (3-10).

$$
\begin{aligned}
& P_{3}^{\prime} P_{2}^{\prime}=x_{3}^{\prime}-x_{1}^{\prime}=k f \frac{x_{3}}{d}-k f \frac{x_{1}}{d}=k f \frac{x_{3}-x_{1}}{d}=k f \frac{P_{3} P_{2}}{d} \\
& P_{2}^{\prime} P_{1}^{\prime}=y_{2}^{\prime}-y_{1}^{\prime}=l f \frac{y_{2}}{d}-l f \frac{y_{1}}{d}=l f \frac{y_{2}-y_{1}}{d}=l f \frac{P_{2} P_{1}}{d}
\end{aligned}
$$


The two scale factors $k$ and $l$ are constants and must be determined first. We know that the object at the edge of the view field of the camera will be projected to the edge of the image film. Therefore, given the focal length $f=4 \mathrm{~mm}$ (no zooming) and the corresponding field of view of the camera $\left(47.5^{\circ}\right.$, horizontal) [58], the horizontal scale factor can be calculated using simple triangle equations.

$$
\frac{160}{4}=k \operatorname{tg} \frac{47.5^{\circ}}{2} \Rightarrow k=91
$$

The vertical scale factor is assumed to be equal to the horizontal scale factor, that is

$$
l=91
$$

In practice, the origin of the image frame is not at the center of the image frame but at the corner of the image frame, for example $O^{\prime \prime}$ in Figure 3.13. As mentioned before, the width and the height of the image frame are 320 pixels and 240 pixels respectively. Then Equations (3-9) and (3-10) are modified as

$$
\begin{aligned}
& x_{1}^{\prime}=364 \frac{x_{1}}{d}+160 \\
& y_{1}^{\prime}=364 \frac{y_{1}}{d}+120
\end{aligned}
$$

According to Equations (3-15) and (3-16), if the coordinate of the human candidate in the world plane is known, the image coordinate of the human candidate is determined. The laser range finder is used to detect the human legs and will give the coordinate of the person in the laser frame. When the coordinate of the person in the laser frame is given, the world plane is determined, which is the plane parallel to the image plane and containing the point that is the location of the human candidate. The purpose is 
to find out the location of each rectangle in the image frame based on the location of each human candidate in the laser frame.

\subsubsection{Center of the Human Candidate Rectangle}

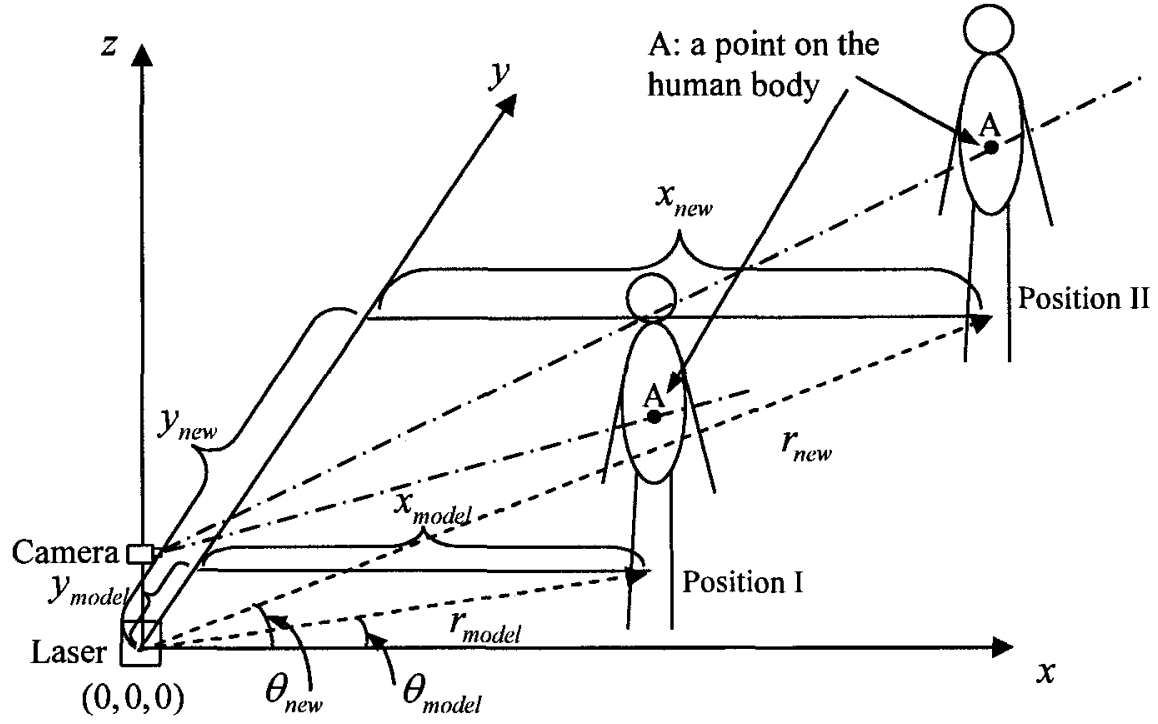

(a) The robot coordinate system

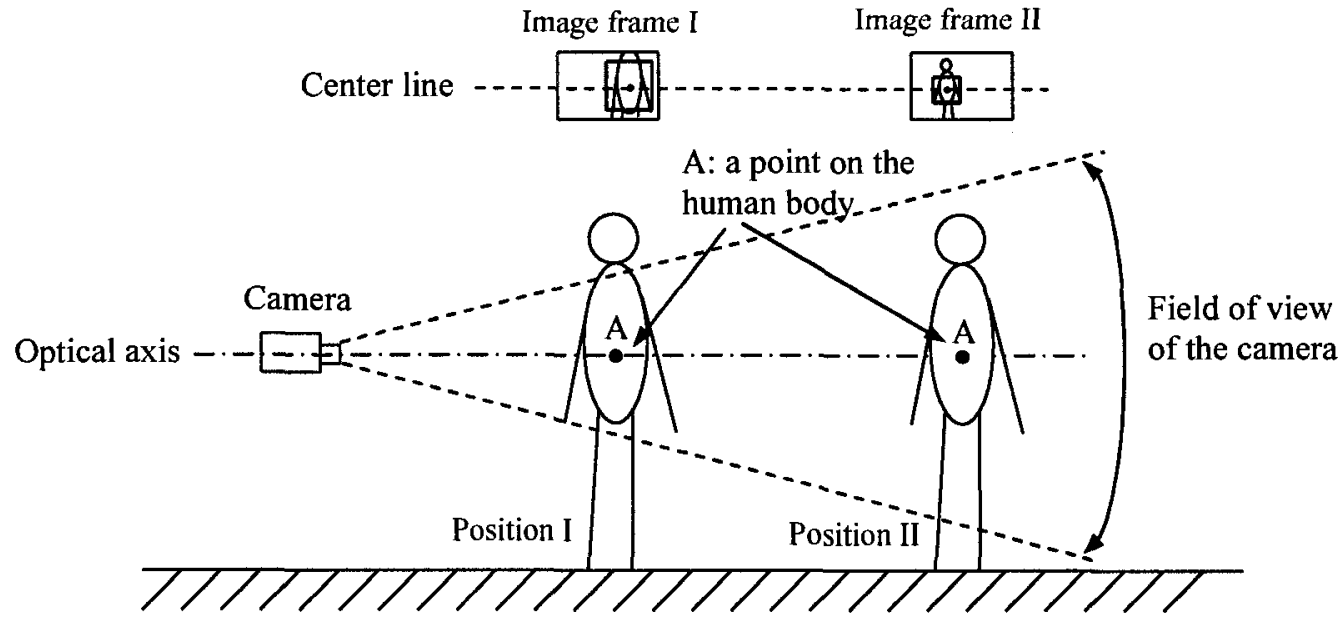

(b) The points on optical axis plane

Figure 3.14. Laser frame to image frame coordinates transformation

Figure 3.14 (a) gives an abstract view of the camera and laser range finder layout of the robot. Assume that the target person model is constructed when the person is at 
Position I and then he/she walks to Position II. Figure 3.14 (b) describes the same scenario but from the point of view of the camera, and Image frames I and II are the ones when the person is at Positions I and II respectively. The laser range finder measures the distance and angle between the person and the robot, while the camera captures one picture at each laser scan. The origin of the laser measurement system is the laser range finder and the laser readings of each laser scan are represented in the polar coordinate system.

Given the polar coordinate of the human candidate at Position II by the laser range finder in Figure 3.14 (a), which is determined by the human candidate detection module, the corresponding Cartesian coordinate of the human candidate is calculated as

$$
\left\{\begin{array}{l}
x_{n e w}=r_{n e w} \cos \theta_{n e w} \\
y_{n e w}=r_{n e w} \sin \theta_{n e w}
\end{array}\right.
$$

where $r_{\text {new }}$ and $\theta_{\text {new }}$ are the distance and the angle between the person and the laser range finder at Position II, and $x_{n e w}$ and $y_{\text {new }}$ are the vertical and horizontal distances between the person and the laser range finder respectively.

Comparison of Figure 3.13 to Figure 3.14 (a), shows that $y_{\text {new }}$ in Equation (3-17) corresponds to $x_{1}$ in Equation (3-15) and $x_{n e w}$ in Equation (3-17) corresponds to $d$ in Equation (3-15). Therefore, the horizontal center of the rectangle in the image frame can be obtained using Equation (3-15). Furthermore, we need to find the vertical center of the rectangle. The vertical center may move up or down in the image frame when the person is walking closer or further. In order that the person is in the center of the image, the camera must tilt accordingly, which makes the localization unstable and complicated. An efficient localization solution is desired. 
In the application of human tracking, the person is supposed to walk on the flat ground. Therefore it is reasonable to assume that the vertical translation of the target person is zero. Since the points on the optical axis of the camera lens will be projected to the center of the imaging surface, all the points on the horizontal plane that contains the optical axis will be projected to the horizontal centre line of the imaging surface if the optical axis of the camera is parallel to the ground,. For simplicity, this plane is referred to as the optical axis plane. Figure 3.14 (b) can be used to explain it further.

In Figure 3.14 (b), when the person is closer to the camera, for example at Position I, point A on the person's body, which is on the optical axis plane, will be projected onto the horizontal centre line of Image frame I; when the person moves to Position II, point A will still be on the horizontal centre line of the new image frame, which is Image frame II. If we can find the points on the human body, which are on the optical axis plane, these points can help us to locate the person in the image. The robot would keep the person within certain distance for the tracking purpose, for example $1.5 \mathrm{~m}$, and the camera is mounted on the robot at $1 \mathrm{~m}$ above the ground. As a result, only part of the human body will be shown in the image. So a practical way to find the vertical center of the target model rectangle in the image is to have it on the horizontal center line of the image. In this way, the vertical center of each rectangle in the following frames is fixed on the horizontal center line of the image. In Figure 3.14 (b), the rectangle image region that represents the person is marked by a thick rectangle in Image frames I and II. The rectangle region in Image frame I, which is for constructing the target person model, has the vertical center on the horizontal center line of the image. Therefore, the rectangle in 
Image frame II has the vertical center on the horizontal center line of the image as well, but with different horizontal center and different size from the rectangle in Image frame I.

\subsubsection{Size of the Human Candidate Rectangle}

For the purpose of target person verification, the rectangle region of a person in different image frames should represent the same part of the person's body no matter how far away the target person is from the camera. For example, in Figure 3.14 (b) the rectangle region in Image frame II contains the same part of the human body as the rectangle region in Image frame I does. The result is that the width and height of the interested part of the person are constants in the world plane. According to Equations (311) and (3-12), the width and height of the rectangle in the image plane are inversely proportional to the perpendicular distance between the world plane and the camera lens. Thus, the relationship between the size of the rectangle of the target model and the size of the rectangle of each human candidate can be determined.

\subsubsection{Equations for Locating the Human Candidate Rectangle}

Using Figure 3.14, the center, the width and height of each rectangle that represents each human candidate in Image frame II are calculated by

$$
\begin{aligned}
& x_{c}=364 \frac{y_{\text {new }}}{x_{\text {new }}}+160 \\
& y_{c}=y_{\text {cmodel }}=120 \\
& w_{\text {new }}=w_{\text {model }} \frac{x_{\text {nodel }}}{x_{\text {new }}} \\
& h_{\text {new }}=h_{\text {moddl }} \frac{x_{\text {model }}}{x_{\text {new }}}
\end{aligned}
$$


where, $x_{\text {new }}$ and $y_{\text {new }}$ have the same meaning as in Equation (3-17), $x_{c}$ and $y_{c}$ are the center coordinate of the rectangle in Image frame II, $w_{n e w}$ and $h_{n e w}$ are the width and height of the rectangle in Image frame II, $y_{\text {cmodel }}$ is the vertical center of the target model, which is the half height of the image, $w_{\text {model }}$ and $h_{\text {model }}$ are the width and height of the rectangle of the target model, $x_{\text {model }}$ is the perpendicular distance between the robot and the person in the target model construction stage.

Frame II in Figure 3.14 (b) can be used to represent every image frame during the tracking process/stage.

\subsubsection{Effects of Camera Panning}

It has been mentioned in section 3.5.1.2 that the camera does not need to tilt for the human tracking application. However, the robot is easy to loose the target if the camera cannot pan. In order to track the human target, the robot should be able to adjust the pan angle of the camera, making sure that the target is always within the field of view. The parameters of the rectangle when the camera pans are calculated in a similar way to Equations (3-18) to (3-21), in addition to an angle calibration step. Specifically, the steps are as follows:

1. Detecting the person in the laser frame: get $r_{\text {new }}$ and $\theta_{\text {new }}$.

2. Getting the pan angle of the camera $\theta_{\text {camera }}$ from camera control unit supplied by the robot.

3. Using the difference between $\theta_{\text {new }}$ and $\theta_{\text {camera }}$ to calculate $x_{\text {new }}$ and $y_{\text {new }}$.

4. Applying Equations (3-18) to (3-21) to determine the new rectangle in the current image frame using the modified angle. 


\subsubsection{Image Processing}

The colour histogram and edge orientation histogram (see section 3.3.1) of the rectangle image region are built for each human candidate.

\subsubsection{Matching}

The colour histogram and edge orientation histogram of each human candidate are compared with the target person model. A similarity measure is applied to choose the candidate with the smallest distance to the target person model. The distance is evaluated based on the Bhattacharyya coefficient [52]:

$$
d(p, q)=\sqrt{1-\sum_{i=1}^{M} \sqrt{p_{i} q_{i}}}
$$

where $p$ and $q$ are normalized distributions, $d(p, q)$ is the Bhattacharyya coefficient denoting the distance between these two distributions.

The distance between the human candidate and the target person $d_{\text {image }}$ is calculated as the summation of the colour histogram distance $d_{\text {color }}$ and the edge orientation histogram distance $d_{\text {edge }}$,

$$
d_{\text {image }}=d_{\text {color }}+d_{\text {edge }}
$$

where $d_{c o l o r}$ and $d_{\text {edge }}$ are computed using Equation (3-22).

\subsection{Particle Filtering}

The location of a person is detected by the laser range finder based on the leg detection algorithm. The location of each leg is approximated by the midpoint of the leg set and the location of the person is approximated by the midpoint of two legs (two-leg 
pattern) or by the location of one leg (single-leg pattern). Errors are generated by the approximation. Furthermore, the laser range finder measurement itself contains errors. A standard particle filter is used to estimate the state of the target person from the noisy measurement.

\subsubsection{Basics of Particle Filters}

The particle filtering is a Monte Carlo method of Bayesian filtering for accomplishing non-Gaussian and/or non-linear tracking. The main idea of the particle filtering is to represent the posterior distribution of the state of the tracked (or observed) object by a set of weighted samples, or particles. Each particle represents a possible instantiation of the state and the corresponding weight describes the possibility of the instantiation. Therefore the particle representation can maintain multiple hypotheses of the state simultaneously.

Denote the state at time instant $k$ to be $\mathbf{x}_{k}$, which will be estimated based on all the previous and current observations $\mathbf{z}_{1: k}$. To estimate the posterior probability density function $p\left(\mathbf{x}_{k} \mid \mathbf{z}_{1: k}\right)$, a set of random samples (particles) $\mathbf{x}_{k}^{i}, i=1, \ldots, m$, with associated weights $w_{k}^{i}, i=1, \ldots, m$, is constructed. The weights are the possibilities of the possible states, that is $\sum_{i=1}^{m} w_{k}^{i}=1$. The posterior probability $p\left(\mathbf{x}_{k} \mid \mathbf{z}_{1: k}\right)$ can be approximated as a sum of weighted Dirac functions (see Figure 3.15 [60]).

$$
p\left(\mathbf{x}_{k} \mid \mathbf{z}_{1: k}\right) \approx \sum_{i=1}^{m} w_{k}^{i} \delta\left(\mathbf{x}_{k}-\mathbf{x}_{k}^{i}\right)
$$




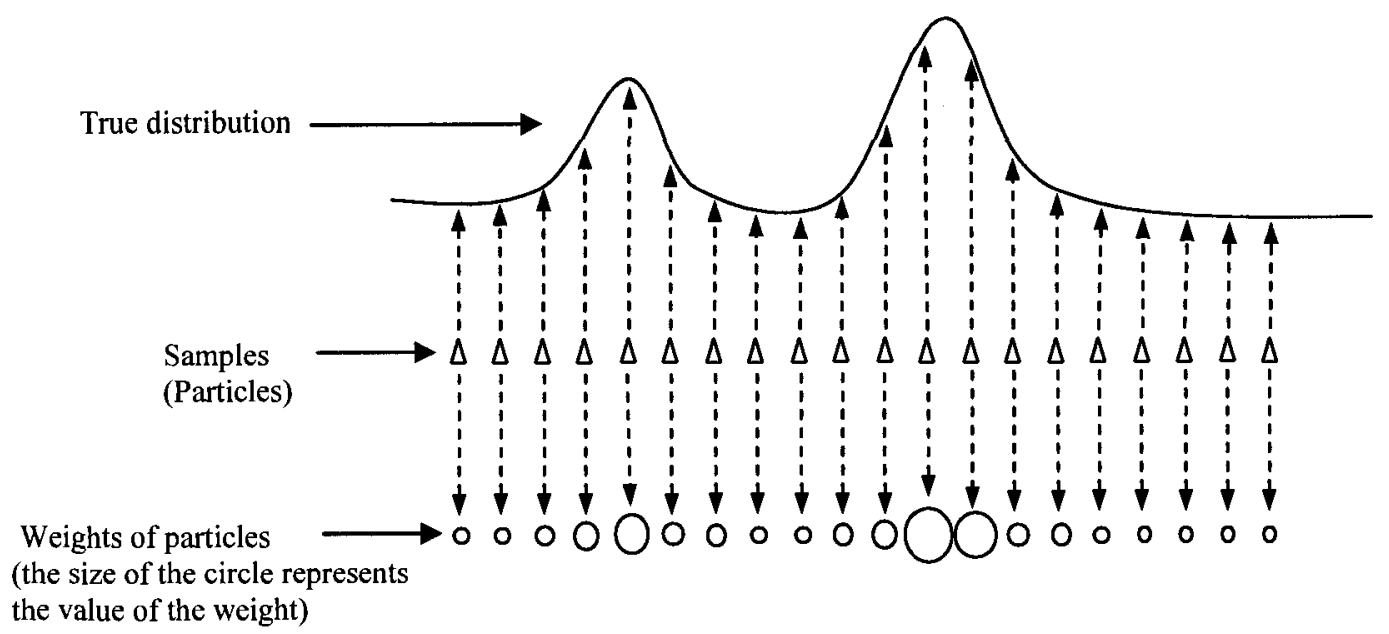

Figure 3.15. Particle representation

From Equation (3-24), we observe that the more the particles (e.g. $m \rightarrow \infty$ ), the closer the right-hand side of the equation is to the true posterior probability density distribution. It is impossible to draw samples from the true posterior distribution directly (Equation (3-24)). Therefore, the weights are obtained by importance sampling [53], which is to draw samples from a proposal function $q(\cdot)$ to make it easier to draw samples. Thus the weight reflects the difference between the proposal and the true posterior probability. Applying the Bayesian rule, the weights $w_{k}^{i}$ in Equation (3-24) is given by

$$
w_{k}^{i} \propto w_{k-1}^{i} \frac{p\left(\mathbf{z}_{k} \mid \mathbf{x}_{k}^{i}\right) p\left(\mathbf{x}_{k}^{i} \mid \mathbf{x}_{k-1}^{i}\right)}{q\left(\mathbf{x}_{k}^{i} \mid \mathbf{x}_{k-1}^{i}, \mathbf{z}_{k}\right)}, i=1, \ldots, m
$$

where $p\left(\mathbf{x}_{k} \mid \mathbf{x}_{k-1}\right)$ is the state transition model, describing the possibility that state $\mathbf{x}_{k-1}$ propagates to $\mathbf{x}_{k}$. Then the filtering procedure is to draw a set of particles from the proposal $q\left(\mathbf{x}_{k} \mid \mathbf{x}_{k-1}, \mathbf{z}_{k}\right)$ and to calculate the weight of each particle according to Equation (3-25) iteratively.

The problem with this filtering procedure is that most of the particles will have weights close to zero after several iterations, which is caused by the increase of weight 
variance over time [53]. This phenomenon is known as the degeneracy problem, which leads to less diversities of the particles and even a failure of tracking because only a few particles, which have larger weights, will contribute to the estimation to the posterior probability. To solve this problem, two methods are usually used. One is drawing particles from a good proposal. The other is discarding the particles with lowest weights by applying a re-sampling function. According to the choice for the proposal, an optimal proposal [38] can be obtained by using

$$
q\left(\mathbf{x}_{k}^{i} \mid \mathbf{x}_{k-1}^{i}, \mathbf{z}_{k}\right)=p\left(\mathbf{x}_{k}^{i} \mid \mathbf{x}_{k-1}^{i}, \mathbf{z}_{k}\right)
$$

In practice, the proposal distribution is chosen to be

$$
q\left(\mathbf{x}_{k}^{i} \mid \mathbf{x}_{k-1}^{i}, \mathbf{z}_{k}\right)=p\left(\mathbf{x}_{k}^{i} \mid \mathbf{x}_{k-1}^{i}\right)
$$

which leads to

$$
w_{k}^{i} \propto w_{k-1}^{i} p\left(\mathbf{z}_{k} \mid \mathbf{x}_{k}^{i}\right)
$$

The re-sampling function generates a new set of particles so that the probability for a particle that is in the old set to be in the new set equals to its weight, which can be viewed as an independent and identically distributed (i.i.d.) sampling procedure. Therefore after re-sampling, the weights are set to $w_{k}^{i}=1 / m$. The re-sampling step is applied at each time instant, which means at time instant $k-1$, we also have $w_{k-1}^{i}=1 / \mathrm{m}$. So Equation (3-28) is simplified as

$$
w_{k}^{i} \propto p\left(\mathbf{z}_{k} \mid \mathbf{x}_{k}^{i}\right)
$$

Equation (3-29) illustrates that the weight can be calculated based on the likelihood of the observation.

The algorithm of the above particle filter is described in Table 3.1. 
Table 3.1. The particle filtering algorithm

1. Initialization

Draw $m$ particles $\mathbf{x}_{0}^{i}$ from the prior distribution $p(\mathbf{x})$, set the weight of each particle $w_{0}^{i}$ to be $1 / m$.

2. State propagation (state prediction)

Based on the transition model, propagate these $m$ particles from the previous state $\mathbf{x}_{k-1}^{i}$ to the current state $\mathbf{x}_{k}^{i}$.

3. Weight computation

Calculate the new weight of each particle using $w_{k}^{i}=p\left(\mathbf{z}_{k} \mid \mathbf{x}_{k}^{i}\right)$

Normalize the weights by $w_{k}^{i}=\frac{w_{k}^{i}}{\sum_{i=1}^{m} w_{k}^{i}}$

4. State estimation

The posterior state $\mathbf{x}_{k}$ is estimated by the minimum mean square error (MMSE) estimation,

$$
\hat{\mathbf{x}}_{k}=E\left[\mathbf{x}_{k} \mid \mathbf{z}_{1: k}\right] \approx \sum_{i=1}^{m} w_{k}^{i} \mathbf{x}_{k}^{i}
$$

5. Re-sampling

The particles with higher weights are duplicated and the particles with lower weights are discarded and keep $m$ new random particles. The weight of each particle $w_{k}^{i}$ is reset to be $1 / m$.

\subsubsection{State Estimation by Particle Filtering}

The particle filtering module is used to estimate the location and velocity of the target person.

\subsubsection{State Description}

The state vector of the target person at time $k$ is chosen as $\mathbf{x}=\left[x, y, v_{x}, v_{y}\right]^{T}$ (for simplicity, the subscript $k$ is omitted), where $(x, y)$ is the location of the person relative 
to the robot and $\left(v_{x}, v_{y}\right)$ are the velocities of the person in $x$ direction and $y$ direction (see Figure 3.16) respectively. It is noted that $(x, y)$ are determined by the initial location $\left(x_{0}, y_{0}\right)$ and velocities $\left(v_{x}, v_{y}\right)$.

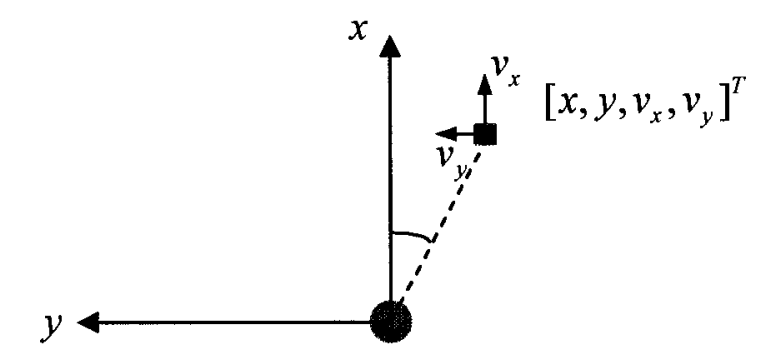

- The person

Figure 3.16. The state vector for the moving person

\subsubsection{Transition Model}

The particles propagate from the previous state to the current state according to a transition model. Considering that the human movement is highly non-linear, the human movement is modeled as a random walking process, which is

$$
\left\{\begin{array}{l}
v_{x k}=\operatorname{RandomGaussian}\left(0, \sigma_{v x}\right) \\
v_{y k}=\operatorname{RandomGaussian}\left(0, \sigma_{v y}\right) \\
x_{k}=x_{k-1}+v_{x k} \Delta T \\
y_{k}=y_{k-1}+v_{y k} \Delta T
\end{array}\right.
$$

where $\sigma_{v x}$ and $\sigma_{v y}$ are the standard deviations of the random Gaussian distribution of $v_{x k}$ and $v_{y k}$, and $\Delta T$ is the time interval between two observations. As indicated in Equation (3-30), the velocities $v_{x k}$ and $v_{y k}$ are drawn from zero-mean random Gaussian distributions. The reason that the velocity is modeled as a zero-mean random Gaussian in 
each time step is that our experiments show that it is the best way to model the human's random movement or sudden stop.

\subsubsection{Ego-Motion Compensation}

The propagation step (Equation (3-30)) involves the states in times $k$ and $k-1$. Because the robot is also moving, which is referred to as ego-motion in this thesis, when following the person, these two states may not be in the same coordinate system. So an ego-motion compensation procedure must be applied before the propagation step. Specifically, the coordinate $(x, y)$ in time $k-1$ is transformed to time $k$ according to the odometer readings indicating the position and heading of the robot. The theory of the coordinate transformation between two time instants is shown in Figure 3.17.

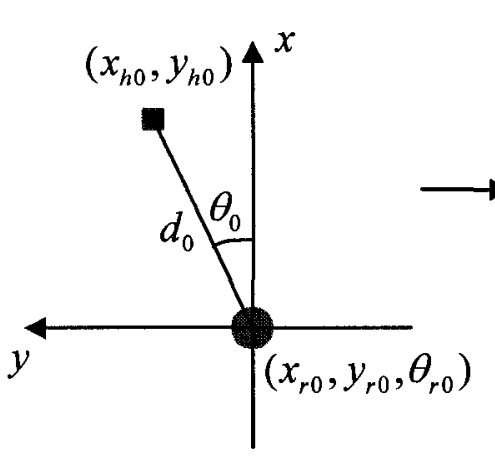

(a)

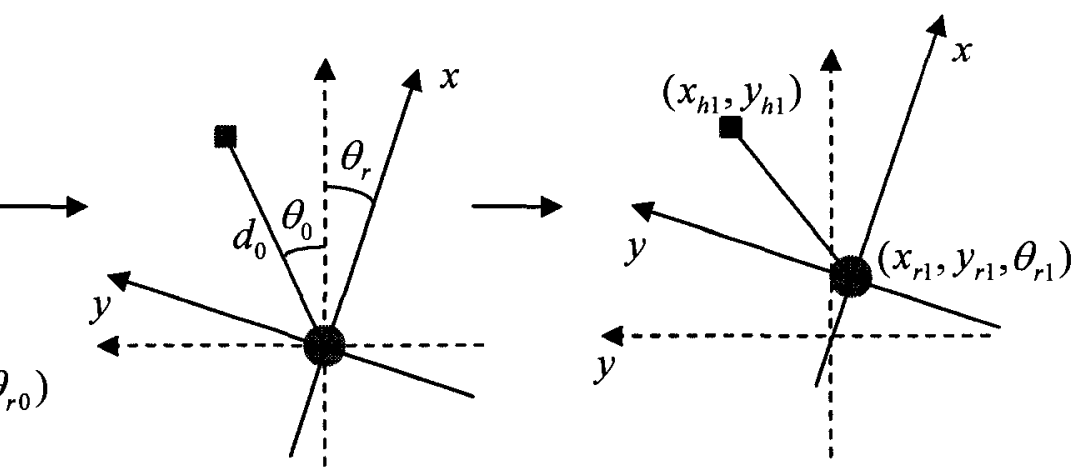

(b)

(c)

Figure 3.17. Ego-motion compensation

In Figure 3.17, the person (represented by the red rectangle) keeps still and the robot (represented by the blue circle) is moving. $\left(x_{h 0}, y_{h 0}\right)$ is the coordinate of the person when the pose of the robot is $\left(x_{r 0}, y_{r 0}, \theta_{r 0}\right)$, which means the position of the robot is $\left(x_{r 0}, y_{r 0}\right)$ and the heading is $\theta_{r 0}$ (Figure $\left.3.16(\mathrm{a})\right) .\left(x_{h 1}, y_{h 1}\right)$ is the new coordinate of the 
person when the robot's pose is $\left(x_{r 1}, y_{r 1}, \theta_{r 1}\right)$ (Figure $\left.3.17(\mathrm{c})\right)$. The robot moves from $\left(x_{r 0}, y_{r 0}\right)$ to $\left(x_{r 1}, y_{r 1}\right)$ with a heading $\theta_{r}=\theta_{r 1}-\theta_{r 0}$.

The purpose is to find out $\left(x_{h 1}, y_{h 1}\right)$ from the given $\left(x_{h 0}, y_{h 0}\right),\left(x_{r 0}, y_{r 0}, \theta_{r 0}\right)$, and $\left(x_{r 1}, y_{r 1}, \theta_{r 1}\right)$. First a rotation (Figure $\left.3.17(\mathrm{~b})\right)$ is performed by which the robot rotates to the desired heading; then a translation is followed by which the robot moves to the desired position (Figure 3.17 (c)). The coordinate of the person $\left(x_{h 1}, y_{h 1}\right)$ is obtained by

$$
\left\{\begin{array}{l}
d_{0}=\sqrt{\left(x_{h 0}^{2}+y_{h 0}^{2}\right)} \\
\theta_{0}=\arctan \left(\frac{y_{h 0}}{x_{h 0}}\right) \\
\theta_{r}=\theta_{r 1}-\theta_{r 0} \\
x_{h 1}=d_{0} \times \cos \left(\theta_{0}+\theta_{r}\right)-\left(x_{r 1}-x_{r 0}\right) \\
y_{h 1}=d_{0} \times \sin \left(\theta_{0}+\theta_{r}\right)-\left(y_{r 1}-y_{r 0}\right)
\end{array}\right.
$$

In Equation (3-31), $\left(x_{r 0}, y_{r 0}, \theta_{r 0}\right)$ and $\left(x_{r 1}, y_{r 1}, \theta_{r 1}\right)$ are obtained from the odometer of the robot. Generally speaking, however, the odometer readings of the mobile robot cannot be used to give the real position of the robot directly because of the wheel slippage. The odometer error resulted from the wheel slippage will accumulate over time without an upper bound. By applying Equation (3-31), the odometer error is limited within one time step. Observe Equation (3-31), we can find that only the difference of the two readings from the robot odometer is used, that is $\left(\theta_{r 1}-\theta_{r 0}\right),\left(x_{r 1}-x_{r 0}\right)$, and $\left(y_{r 1}-y_{r 0}\right)$. In other words, each previous robot pose $P_{p}$ can be assumed to be $(0,0,0)$. When the robot moves to the current pose $P_{c}$, the odometer error only accumulate within one time step. 


\subsubsection{Particles Initialization}

The location of the person obtained by the laser range finder in the target model construction state is input to the particle filter to initialize $(x, y)$ of the $m$ particles. The velocities $\left(v_{x}, v_{y}\right)$ of the initial particles are set to $0 \mathrm{~s}$.

\subsubsection{Particles Propagation}

The state of the target person in time $k-1$ is propagated to the next state in time $k$ using Equation (3-30) after the ego-motion compensation (Equation (3-31)) procedure is finished.

\subsubsection{Weight Computation}

The weight of each particle is calculated using Equation (3-29), which is the likelihood distribution. Two features are used to evaluate the weight: the distance and the angle between the target person and the robot. The likelihood distribution of the distance $p_{1}(\mathbf{z} \mid \mathbf{x})$ is calculated as

$$
p_{1}(\mathbf{z} \mid \mathbf{x})=e^{-\left(d_{l}-d_{p}\right)^{2} / \sigma_{d}^{2}}
$$

where $\sigma_{d}^{2}$ is the covariance, $d_{l}$ is the distance between the robot and the person measured by the laser range finder in the current time step, $d_{p}$ is the distance between the robot and each particle person, which is calculated by the $(x, y)$ components in the state vector.

$$
d_{p}=\sqrt{x^{2}+y^{2}}
$$

The likelihood of the angle $p_{2}(\mathbf{z} \mid \mathbf{x})$ is calculated as

$$
p_{2}(\mathbf{z} \mid \mathbf{x})=e^{-\left(A_{l}-A_{p}\right)^{2} / \sigma_{A}^{2}}
$$


where $\sigma_{A}^{2}$ is the covariance, $A_{1}$ is the angle between the robot and the person measure by the laser range finder in the current time step, $A_{p}$ is the angle between the robot and the particle person, which is calculated by

$$
A_{p}=\arctan \left(\frac{y}{x}\right)
$$

Suppose the distance and the angle are two independent variables, the weight of the $i^{\text {th }}$ particle is then calculated as the product of the two likelihoods.

$$
w^{i}=p_{1} p_{2}=e^{-\left(d_{i}-d_{p}\right)^{2} / \sigma_{d}^{2}-\left(A_{i}-A_{p}\right)^{2} / \sigma_{t}^{2}}
$$

And the weights are normalized by

$$
w^{i}=\frac{w^{i}}{\sum_{i=1}^{m} w^{i}}
$$

where $m$ is the total number of particles.

\subsubsection{State Estimation and Re-sampling}

The estimated posterior state $\left[\hat{x}, \hat{y}, \hat{v}_{x}, \hat{v}_{y}\right]^{T}$ at time $k$ is computed by (the subscript $k$ is omitted)

$$
\left\{\begin{array}{l}
\hat{x}=\sum_{i=1}^{m} w^{i} x^{i} \\
\hat{y}=\sum_{i=1}^{m} w^{i} y^{i} \\
\hat{v}_{x}=\sum_{i=1}^{m} w^{i} v_{x}{ }^{i} \\
\hat{v}_{y}=\sum_{i=1}^{m} w^{j} v_{y}{ }^{i}
\end{array}\right.
$$


The particles with higher weights are multiplied and the particles with lower weights are discarded to generate a new set of $\mathrm{m}$ particles using a re-sampling step. The weights of the new set are normalized to 1 .

\subsubsection{Tracking Failure}

In cases that the detection of the target person fails (e.g., the leg detection module fails to detect a human leg or the target person walks away from the field of view of the camera), a state maintenance step is added to the tracking stage. The tracking will be resumed as soon as the robot successfully detects the target person in the field of view again.

\subsection{Robot Control}

According to the state of the target person estimated by the particle filter (Equation (3-38)), the distance $r_{h}$ and angle $\theta_{h}$ between the person and the robot, and the velocity $v_{h}$ of the person are calculated as

$$
\left\{\begin{array}{l}
r_{h}=\sqrt{\hat{x}^{2}+\hat{y}^{2}} \\
\theta_{h}=\arctan \left(\frac{\hat{y}}{\hat{x}}\right) \\
v_{h}=\sqrt{\hat{v}_{x}^{2}+\hat{v}_{y}^{2}}
\end{array}\right.
$$

If the distance $r_{h}$ is less than a threshold, the robot will only turn with angle $\theta_{h}$ and no translation. If the distance $r_{h}$ is larger than a threshold, which means the person is walking further away from the robot, the robot will move with heading $\theta_{h}$ and velocity $v_{h}$ in order to keep following the target within a predefined range of distance. 


\section{Chapter 4 System Implementation}

In this chapter, the tracking scheme developed in Chapter 3 is implemented on a Performance PeopleBot ${ }^{\mathrm{TM}}$ robot manufactured by ActiveMedia Inc. As mentioned in chapter 3 , the human tracking robot system is composed of modules of target person model construction, human candidate detection, target person verification, particle filtering, and robot control. The implementation details of each module are discussed.

\subsection{Robot Configuration}

The Performance PeopleBot ${ }^{\mathrm{TM}}$ robot, a product of ActiveMedia Inc, is equipped with a SICK LMS200 laser range finder, two sonar sensor rings on the top deck and the bottom deck respectively, a PTZ CANON VC-C4 camera mounted on a pan-tilt unit, wheels, and an onboard computer with a touch screen. The processor of the onboard computer is Pentium III, $850 \mathrm{MHz}$ and the RAM is $256 \mathrm{MByte}$. The operating system of the onboard computer is Linux Red Hat 7.2. The human tracking program is developed using $\mathrm{C}++$ on the onboard computer with the help of OpenCV [55] and ARIA (the short for ActivMedia Robotics Interface for Application) [56]. OpenCV is a C++ open source library for computer vision developed by Intel. ARIA is the $\mathrm{C}++$ based open source library supplied by the robot company. It provides the interface between robot sensors and controllers. 


\subsubsection{The Laser Range Finder}

The robot controller communicates with the onboard computer in the following way: commands are sent from the onboard computer to the robot controller and the sensor data, such as the data from the laser range finder, the sonar, and the odometer, are collected by the robot controller and sent from the robot controller to the onboard computer in each robot cycle (the same cycle for the particle filtering). For determining the communication cycle between the robot controller and the onboard computer, the specifications of the laser range finder (Table $4.1[57,59])$ are explored.

Table 4.1. Specifications of the laser range finder

\begin{tabular}{|l|l|}
\hline Scanning Angle & $100^{\circ} / 180^{\circ}$ \\
\hline Angular Resolution & $0.25^{\circ}, 0.5^{\circ}, 1^{\circ}$ for $100^{\circ} ; 0.5^{\circ}, 1^{\circ}$ for $180^{\circ}$ \\
\hline Resolution/Typical Measurement Accuracy & $10 \mathrm{~mm} / \pm 15 \mathrm{~mm}$ \\
\hline Typical Range & $10 \mathrm{~m}$ \\
\hline Scan Frequency & $75 \mathrm{~Hz}$ \\
\hline Data Form & 1 start bit, 8 data bits, 1 stop bit (no parity) \\
\hline Data Transfer & $38.4 \mathrm{Kbaud}$ \\
\hline Interface & RS-232 serial interface \\
\hline
\end{tabular}

For the human leg detection, the angular resolution of the laser scan is chosen as $0.5^{\circ}$ in the implementation. In ARIA, the scanning angle of the laser range finder can be set to be $180^{\circ}$ (from $-90^{\circ}$ to $+90^{\circ}$ ) or $100^{\circ}$ (from $-50^{\circ}$ to $+50^{\circ}$ ). For the wide angle case $\left(180^{\circ}\right)$, there are $(90-(-90)) \times 2+1=361$ laser readings in one laser frame. For the narrow angle case $\left(100^{\circ}\right)$, there are $(50-(-50)) \times 2+1=201$ laser readings in one laser frame. Each laser reading contains two bytes: one high byte for the distance between the reflection 
point and the center of the laser range finder and one low byte for the angle between the reflection point and the center of the laser range finder. Each byte is transferred with the data form in Table 4.1. So the total bits of one laser frame are:

$$
\begin{aligned}
& 180^{\circ}: \quad 361 \times 2 \times 10=7220 \text { bits } \\
& 100^{\circ}: \quad 201 \times 2 \times 10=4020 \text { bits }
\end{aligned}
$$

Except for several serial communication control bytes, the transmission time for the data being transferred from the laser range finder to the onboard computer is at least (using the data transfer rate in Table 4.1)

$$
\begin{aligned}
& 180^{\circ}: \frac{7220 \text { bits }}{38.4 \times 10^{3} \text { bits } / \mathrm{s}}=188.02 \mathrm{~ms} \\
& 100^{\circ}: \quad \frac{4020 \text { bits }}{38.4 \times 10^{3} \mathrm{bits} / \mathrm{s}}=104.69 \mathrm{~ms}
\end{aligned}
$$

Recall that for the particle filtering in section 3.6.2.2, the movement of the observed person is modeled as a zero-mean random process, which means the person is expected to have slight translation in two consecutive laser frames, therefore a short cycle (or time step) for particle filtering is desired. Furthermore, in the human tracking application, the target person is always in the center of the field of view of the laser range finder (under tracking). So, a narrow laser scanning angle from $-50^{\circ}$ to $+50^{\circ}$ is better for the application (Figure 4.1).

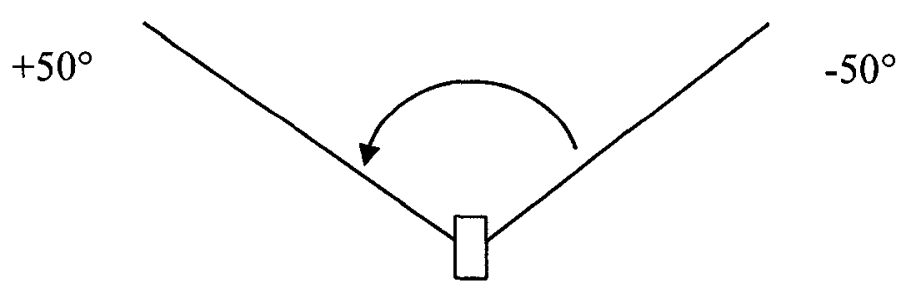

SICK LMS 200

Figure 4.1. Laser scan region 
Based on the discussion above, the communication cycle for the onboard computer to gather data from sensors is set to be $120 \mathrm{~ms}$ (a bit larger than $104.69 \mathrm{~ms}$ ). The particle filter will update its estimation based on the consequence of the laser human leg detection. Thus the time step for the particle filtering equals to $120 \mathrm{~ms}$.

\subsubsection{The PTZ Camera}

The specifications of the Canon VC-C4 camera are given in Table 4.2 [58].

Table 4.2. Canon VC-C4 camera specifications

\begin{tabular}{|l|l|}
\hline Video Standard & NTSC \\
\hline Field of View (Horizontal) & $47.5^{\circ}$ \\
\hline Focal Length & 4 to $64 \mathrm{~mm}$ \\
\hline Zoom & $16 \mathrm{X}$ \\
\hline Pan Angle & $+/-100^{\circ}$ \\
\hline Pan Speed & $1^{\circ}$ to $90^{\circ} / \mathrm{s}$ \\
\hline Tilt Angle & $+90^{\circ} /-30^{\circ}$ \\
\hline Tilt Speed & $1^{\circ}$ to $70^{\circ} / \mathrm{s}$ \\
\hline
\end{tabular}

For the human tracking application, if the field of view of the camera is too narrow, it is easy for the target person to walk out of the reach of the camera, which makes the tracking fail. The largest field of view of the camera, which is $47.5^{\circ}$ (about $23.5^{\circ}$ to $+23.5^{\circ}$, focus length is $4 \mathrm{~mm}$ ), is chosen in the implementation.

\subsubsection{Program Structure}

Two threads are generated to accomplish the human tracking task. One thread is for the camera and the other is for the laser range finder. Note that the human detection and particle filtering are both performed on the laser data, so the laser thread is the main 
loop of the tracking program. As mentioned in section 4.1.1, the laser data will be sent to the onboard computer every $120 \mathrm{~ms}$, i.e., the time interval for the main loop is $120 \mathrm{~ms}$. In each loop, the program will:

1. Process the laser data to extract the human candidates.

2. Verify the target person in the image frame.

3. Generate the moving commands for the robot by the particle filtering.

Since the location of the target person (after verification) is the input to the particle filter, the image processing for target person verification is placed within the laser thread. The major function of the camera thread is to capture images for the construction of target person model (in the pre-tracking stage), so this thread will be actually a void loop in the tracking stage. The images for target person verification will be captured within the laser thread.

The laser data are sampled every $120 \mathrm{~ms}$. Theoretically the image frame, which is used to verify the target person, should be captured at the same time instant as the laser scan starts. In practice, however, it is difficult to know the exact time instant that the laser scan begins. But the difference between the instants for the image grabbing and the laser scan can be reduced as much as possible. To achieve this, an image frame is grabbed right after each laser loop is started. The image frame is stored and will be used in the next laser loop.

The flow chart of the human tracking system is shown in Figure 4.2. The laser range finder and the camera are initialized in the main function and then the camera thread and the laser thread are activated. The camera thread is highlighted with the thin dashed box while the laser thread is highlighted with the thick dashed box. 


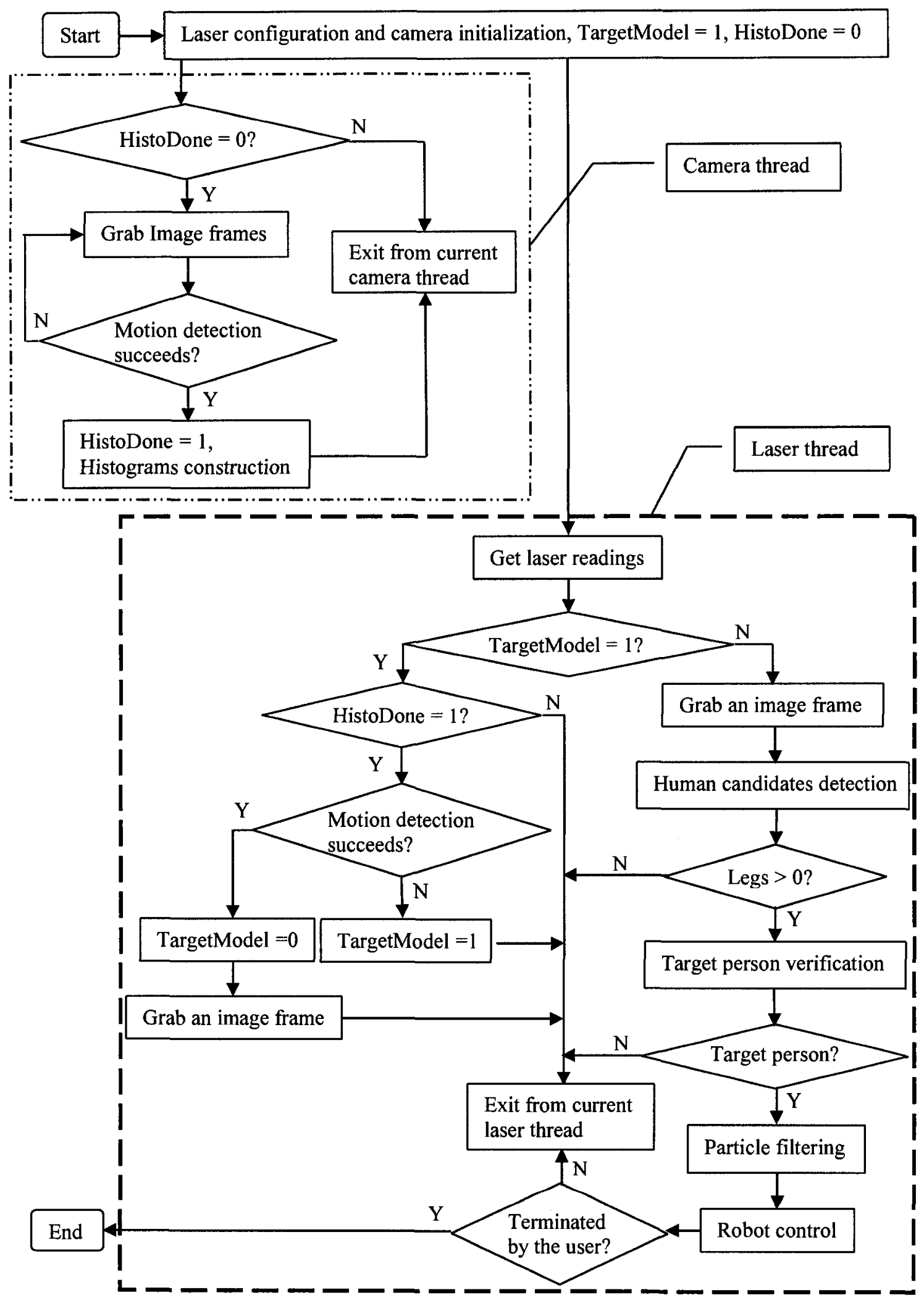

Figure 4.2. Flow chart of the human tracking robot system 


\subsection{Target Person Model Construction}

For building the target person model, the target person is requested to be the only moving object in the field of view of the robot ("seen" by both the laser range finder and the camera) in the pre-tracking stage.

\subsubsection{Image Processing}

The target person is segmented from the background by motion detection. The colour histogram and edge orientation histogram are calculated for the segmented person image.

\subsubsection{Motion Detection}

After the camera initialization (no panning, no tilting, and no zooming), two successive image frames are grabbed from the camera. The two image frames are transformed from 3-channel RGB colour images to intensity images for motion detection. To remove the sensor noise from the intensity images, the two images are smoothed by convoluting with a 3-tap kernel $\left[\begin{array}{lll}0.25 & 0.5 & 0.25\end{array}\right]$. The convolution is taken on the intensity image horizontally and vertically.

The 3-tap kernel acts as a low-pass filter. Suppose $x(n)$ is the input 1D discreet sequence, which stands for the horizontal or vertical 1D image sequence, and $y(n)$ is the output, the frequency response $H(\omega)$ of this 3-tap kernel is obtained by

$$
\begin{gathered}
y(n)=0.25 x(n-1)+0.5 x(n)+0.25 x(n+1) \\
Y(\omega)=0.25 e^{-j \omega} X(\omega)+0.5 X(\omega)+0.25 e^{j \omega} X(\omega) \\
H(\omega)=\frac{Y(\omega)}{X(\omega)}=0.5+0.5 \cos \omega=0.5(1+\cos \omega)
\end{gathered}
$$


The cosine function in Equation (4-1) shows the low-pass property of this 3-tap filter.

The difference image resulted from the two smoothed intensity images is applied with a threshold to remove the pixels with small values. In the implementation, the threshold is set to be 80 out of 255 . The motion region is bounded with a rectangle in the difference image. The difference image generated by a small movement of the human body, for example, hands movement, cannot give an effective representation to the person. So an area threshold is also applied to the bounding rectangle for detecting apparent movement of the human body. If the area of the rectangle is less than the area threshold, two other successive image frames will be grabbed for motion detection. Knowing that the size of the image frame is $320 \times 240$ (width $\times$ height) pixels, the area threshold is set to be

$$
T_{A}=0.4 \times 320 \times 240=30720
$$

The person image is then segmented from the current 3-channel RGB image frame using this bounding rectangle.

\subsubsection{The HS Colour Histogram}

The 2D HS colour histogram introduced in section 3.3.1.1 is implemented. The colour bins for $H$ and $S$ components are chosen as 10 and the brightness bins for $V$ component are chosen to be 8 . The total bins of the colour histogram for the segmented person image are $(10 \times 10+8) \times 2=216$. The $H$ component is scaled from $[0,360]$ to $[0$, $180]$ which occupies one byte in OpenCV. The $S$ component and $V$ component are scaled from $[0,1]$ to $[0,255]$. The pixels with $V<51$ or $S<51$ are assigned to brightness bins. The pixels with $V$ and $S$ above the two thresholds are assigned to colour bins. Figure 4.3 shows an example of the 2D histogram. Figure 4.3 (a) is the current image frame with the 
size of $320 \times 240$ pixels. Figure 4.3 (b) is the segmented person image, in which the size is $142 \times 240$ pixels. Figure 4.3 (c) is the upper part of the image in Figure 4.3 (b), and Figure 4.3 (d) is the normalized sub-histogram for Figure 4.3 (c) with 108 bins. Figure 4.3 (e) is the lower part of the image in Figure 4.3 (b), and Figure 4.3 (f) is the normalized subhistogram for Figure $4.3(\mathrm{e})$ with 108 bins. Figure $4.3(\mathrm{~g})$ is the normalized histogram for the whole segmented person image with 216 bins, in which the sub-histogram of the lower part is concatenated to the sub-histogram of the upper part and the two subhistograms are renormalized to 1 by multiplying 0.5 .

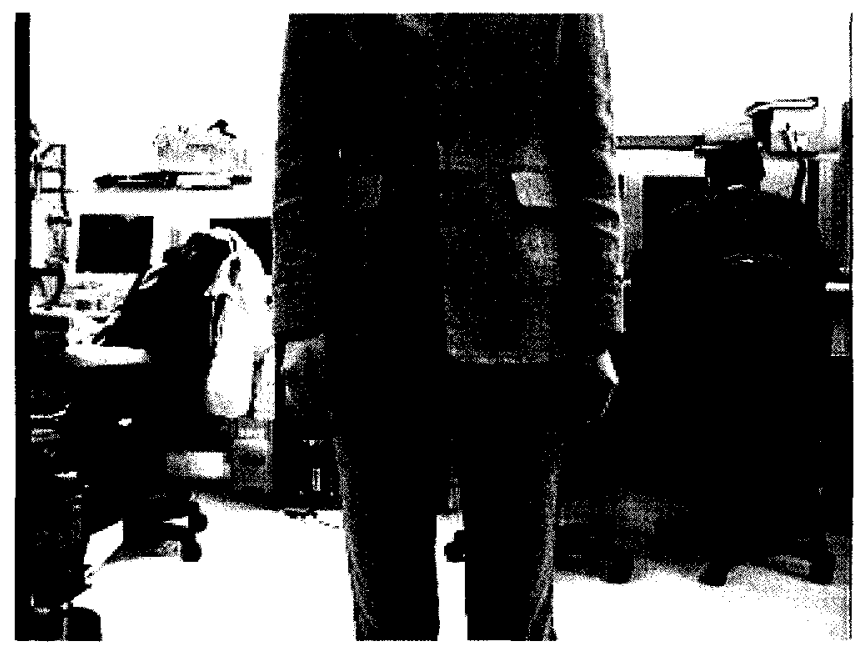

(a) Current image frame

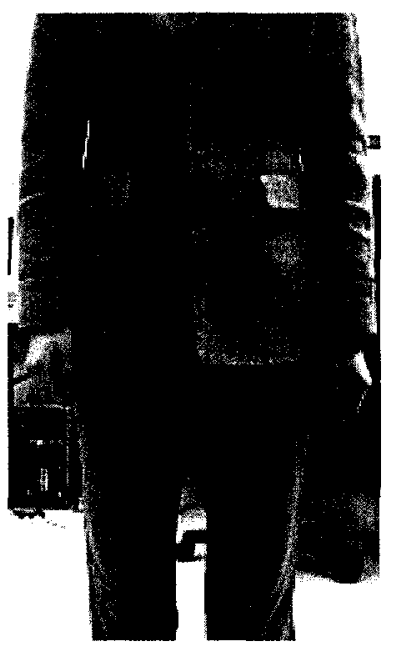

(b) Segmented person image
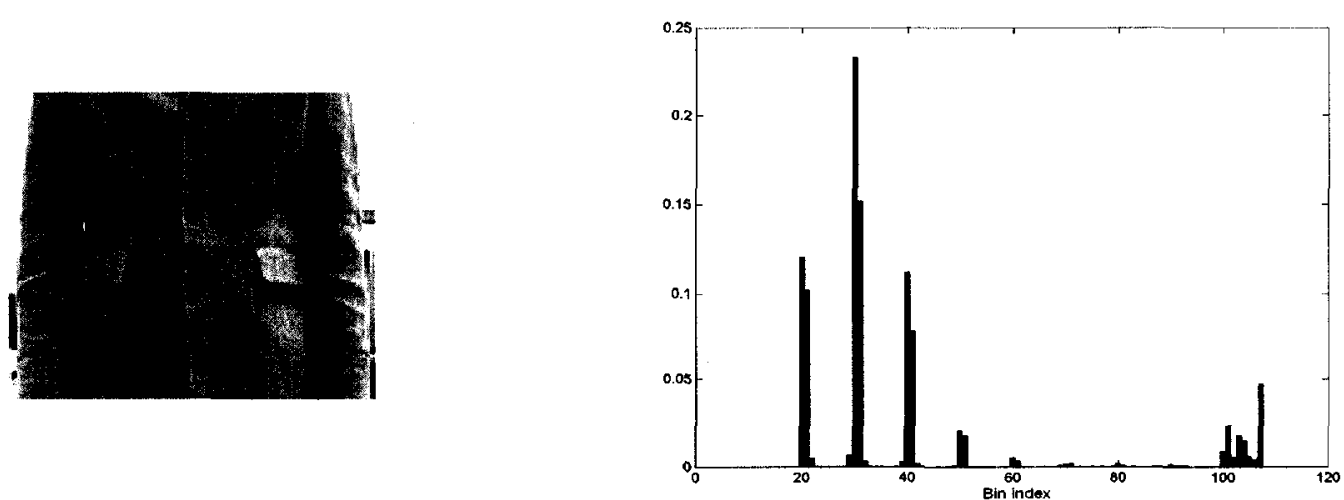

(c) The upper part of (b)

(d) The colour histogram for (c) (108 bins) 

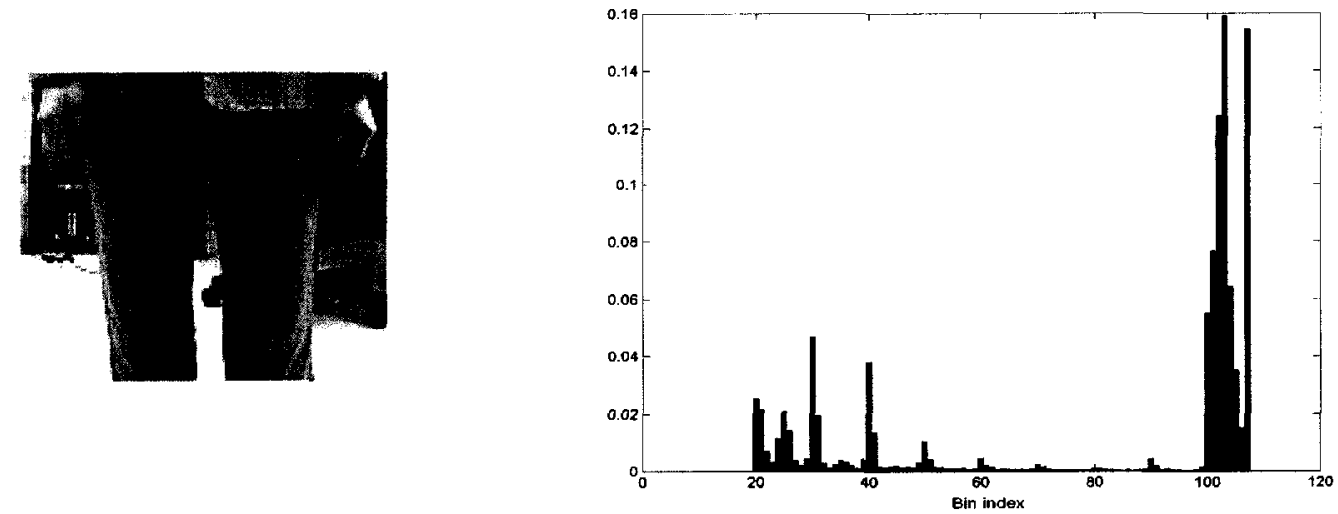

(e) The lower part of (b)

(f) The colour histogram for (e) (108 bins)

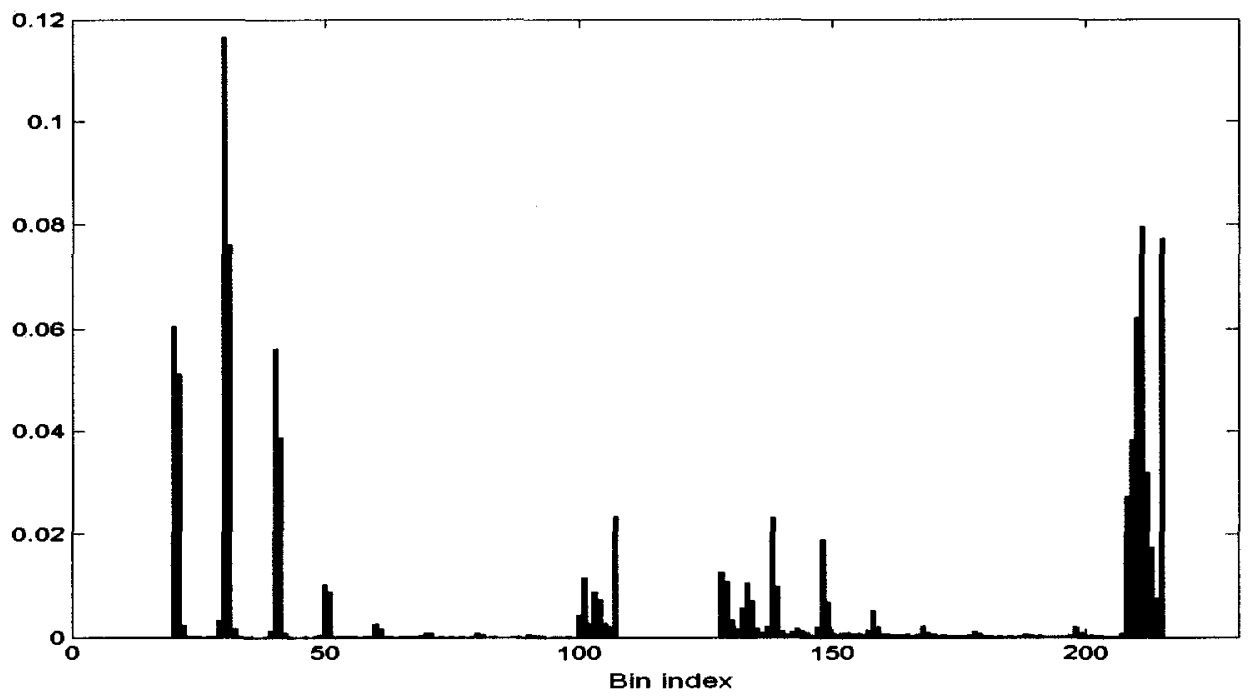

(g) The whole colour histogram for (b) (216 bins)

Figure 4.3. The colour histogram for person 1

Figure 4.4 is the colour histogram of another person. Figure 4.4 (a) is the image frame with the original size and Figure 4.4 (b) is the segmented image with a size of $129 \times 240$ pixels. Other sub-figures in Figure 4.4 have the same meanings as those in Figure 4.3. 


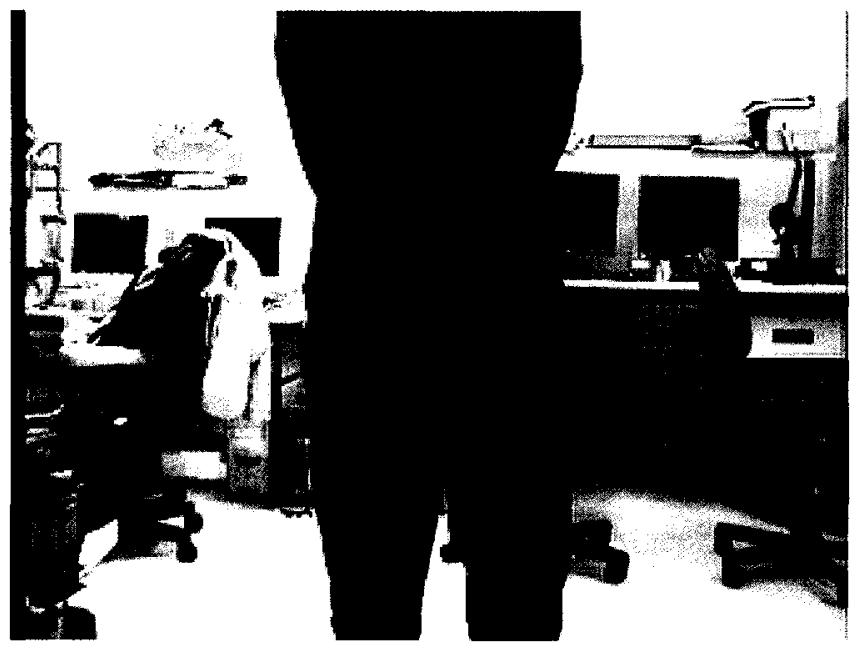

(a) Current image frame

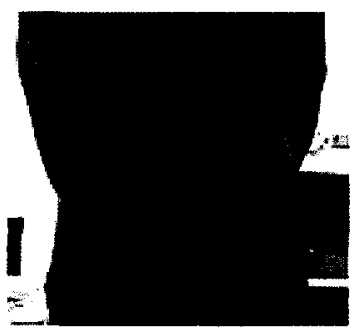

(c) The upper part of (b)

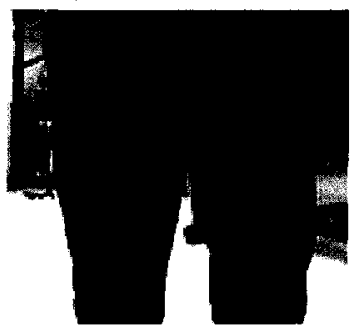

(e) The lower part of (b)

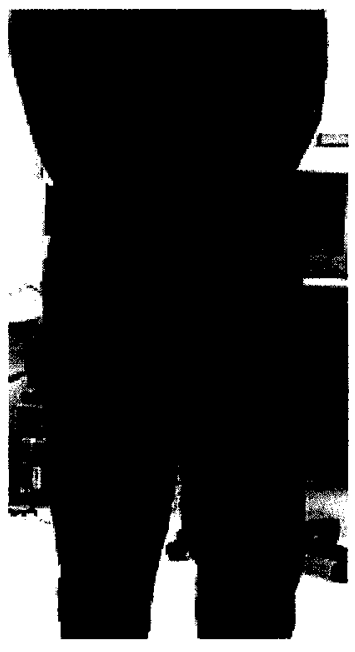

(b) Segmented person image

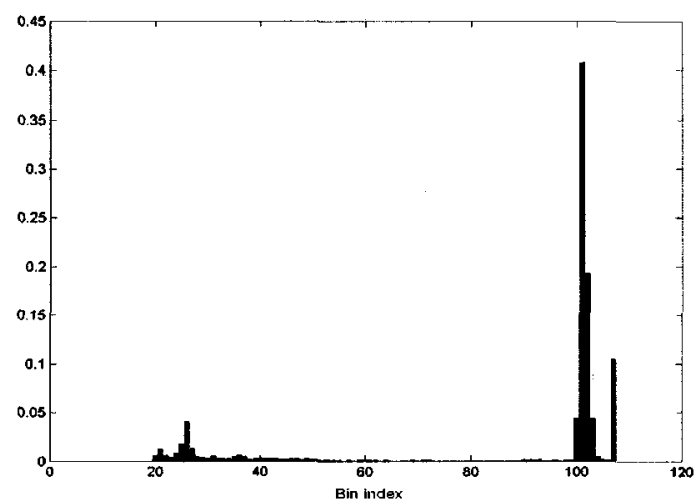

(d) The colour histogram for (c) (108 bins)

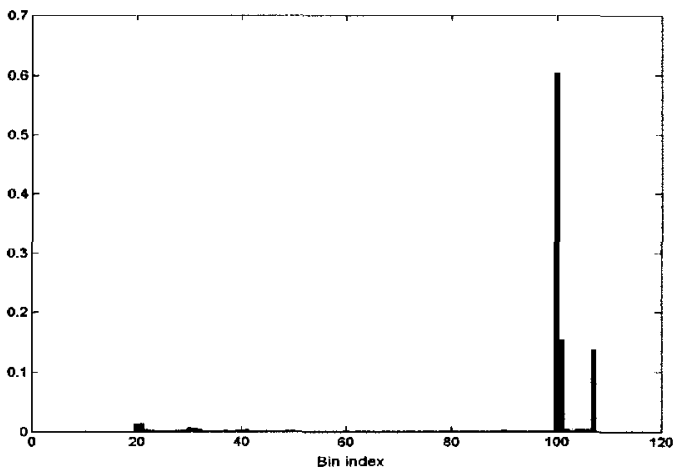

(f) The colour histogram for (e) (108 bins) 


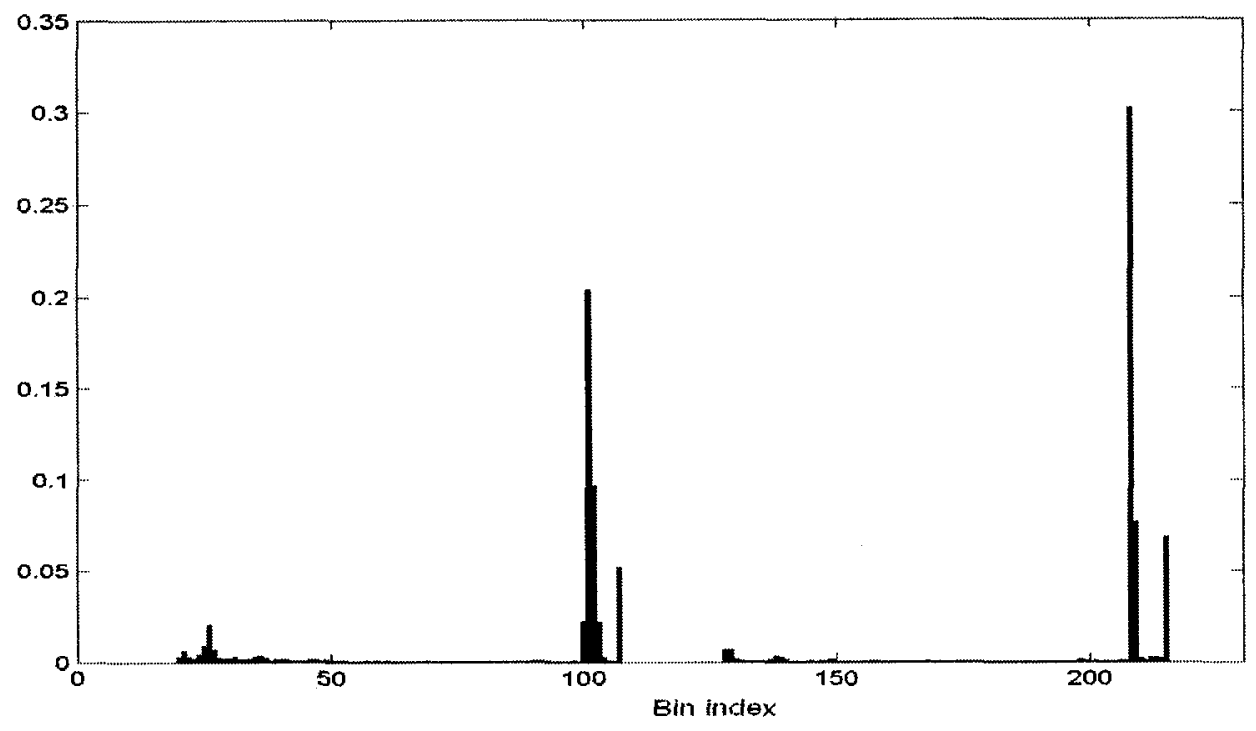

(g) The whole colour histogram for (b) (216 bins)

Figure 4.4. The colour histogram for person 2

Comparing Figure $4.3(\mathrm{~g})$ and Figure $4.4(\mathrm{~g})$, it can be found that the colour distributions of these two persons are obviously different.

\subsubsection{Edge Orientation Histogram}

The edge orientation histogram is built on the whole segmented person image. The number of bins is chosen as 72. The orientation image obtained using Equation (3-5), which is an arctangent function, has the range as $[-\pi,+\pi]$. For simplicity of illustration, the angle range is scale to $[0,2 \pi]$. Then the gap between each bin is $360^{\circ} / 72=5^{\circ}$. Only the strong edges are considered. A threshold for choosing strong edges is set as

$$
0.4 \times \mathrm{Mag}_{\max }
$$

where $\mathrm{Mag}_{\max }$ is the maximum value of the magnitude of the edges (the edge magnitude is computed using Equation (3-4)). 
Figure 4.5 (a) is the edge image of the person and Figure 4.5 (b) shows the corresponding edge orientation histogram. Figure 4.5 (c) is the edge image for the background with no target person in the scene and Figure 4.5 (d) is the edge orientation histogram of Figure 4.5 (c).

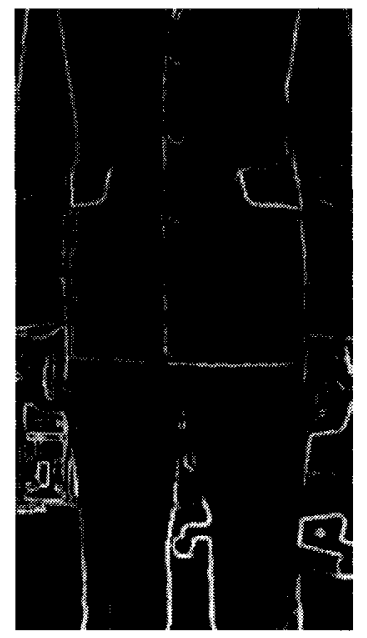

(a) Magnitude image (person)

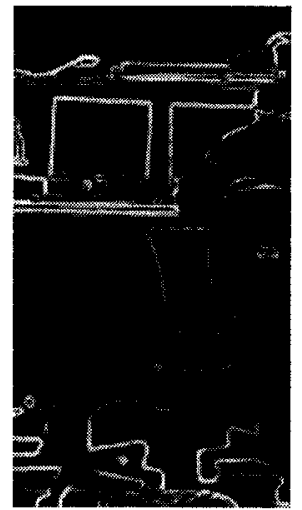

(c) Magnitude image (background)

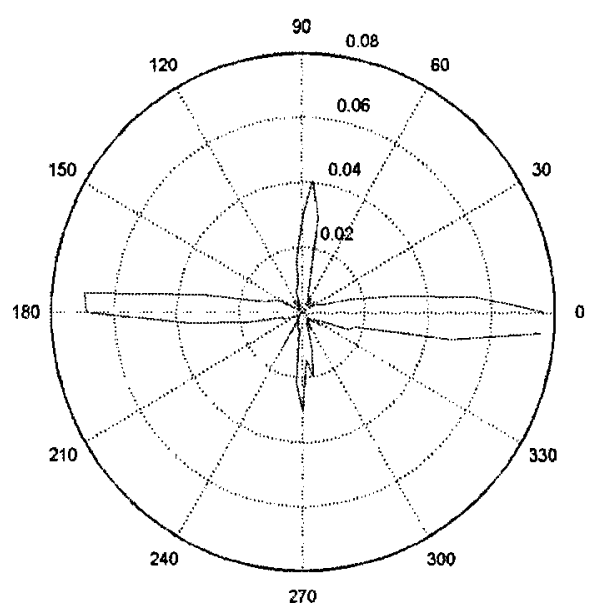

(b) The edge orientation histogram of (a)

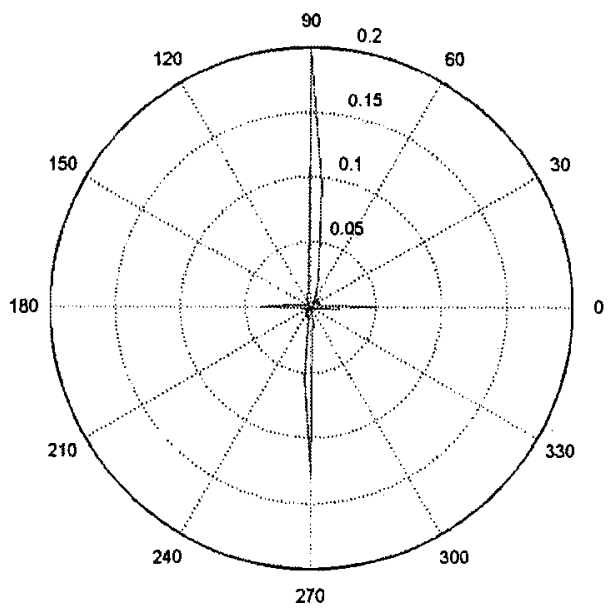

(d) The edge orientation histogram of (c)

Figure 4.5. The edge orientation histogram

The difference between the two edge orientation histograms (Figure 4.5 (b) and Figure 4.5 (d)) is used to distinguish the target person from the background. 


\subsubsection{Location Measurement}

Similar to the motion detection in the image frame, the location of the person can be found by performing motion detection in the laser frame. A laser frame, which is referred as the reference laser frame, is stored in the memory. The leg candidates are generated by using the leg detection criterion 1 and 2 in section 3.4.2. The difference laser frame is generated by subtracting the current laser frame from the reference laser frame. A threshold is used for the difference laser frame to remove the effect of the noise from the laser range finder. The typical measurement accuracy of the laser range finder is $\pm 15 \mathrm{~mm}$ [59], so the threshold for the difference laser frame is set to be $30 \mathrm{~mm}$. If the differences occur within the location of a leg candidate, the location of the target person is obtained and the location will be stored for target person verification. If not, another laser frame will be processed in the next time step, that is, after $120 \mathrm{~ms}$.

\subsubsection{Flow Chart of the Target Person Model Construction}

The flow chart of the target person model construction module is shown in Figure 4.6. 


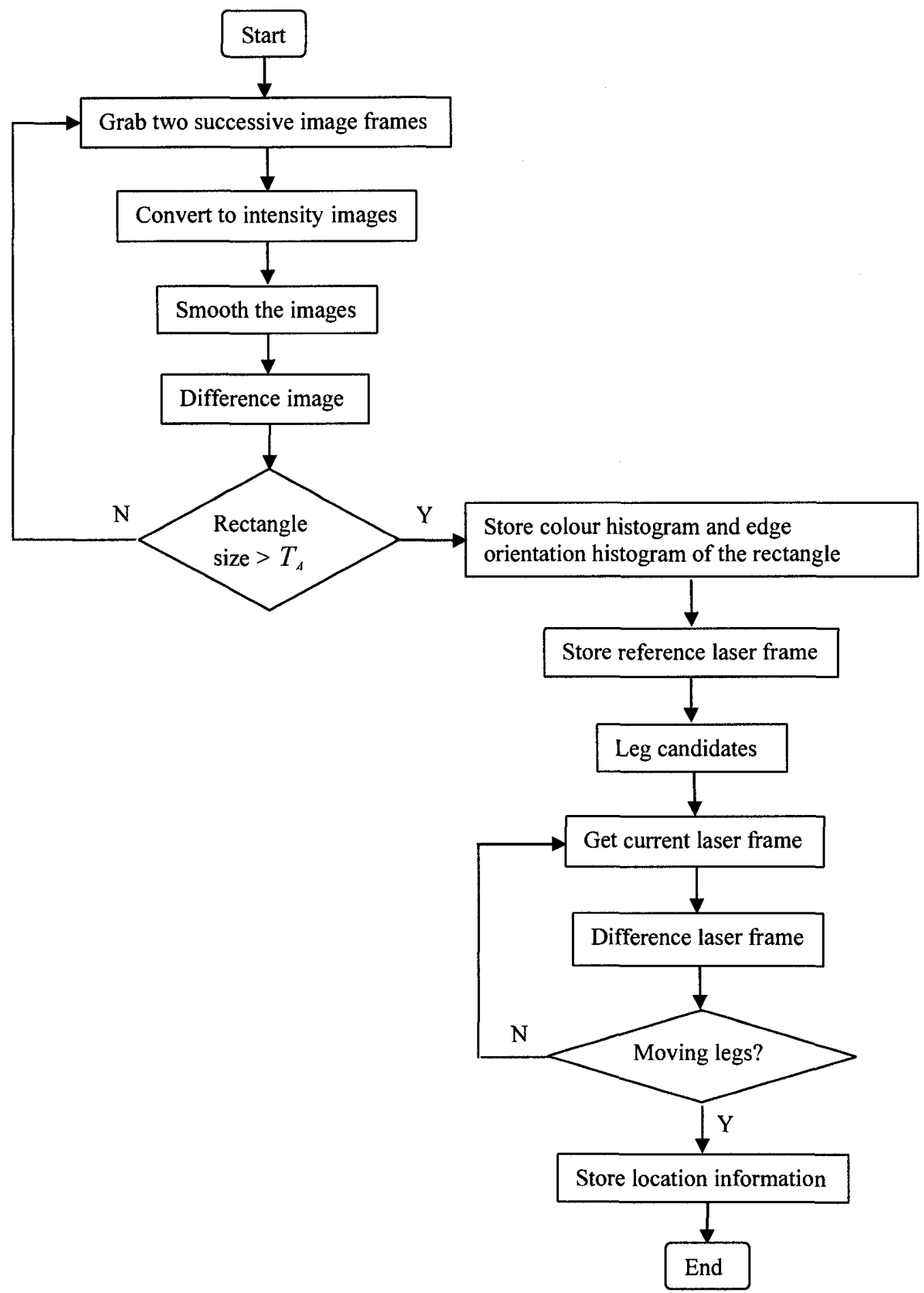

Figure 4.6. Flowchart of target person model construction 


\subsection{Human Detection}

Human detection is performed on the laser frame. Three steps are included in the human detection module: data clustering, leg candidate detection, and leg grouping. The implementation details are described below.

\subsubsection{Data Clustering}

Every $120 \mathrm{~ms}$, one frame of laser readings is obtained from the laser range finder. Each laser reading contains one-byte distance information and one-byte angle information. For simplicity, the distance larger than $3.5 \mathrm{~m}$ is set to be $3.5 \mathrm{~m}$. An example of the laser scan in a polar coordinate system is shown in Figure 4.7. The laser range finder is in the center of the semi-circle in Figure 4.7.

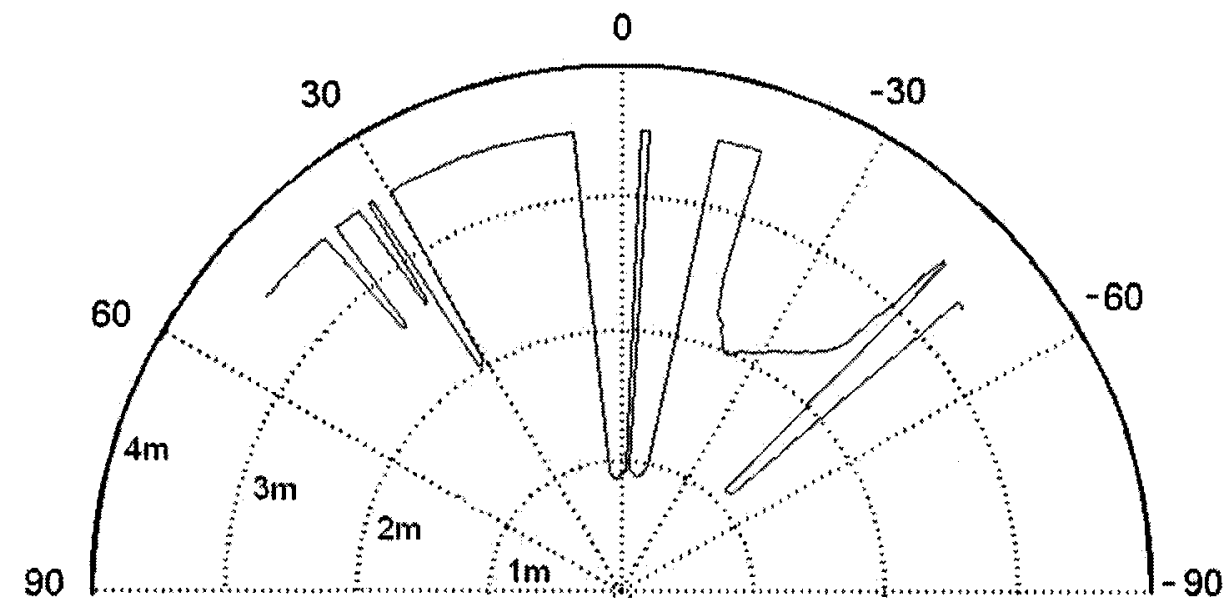

Figure 4.7. A laser scan example in the polar coordinate system

In data clustering, the laser readings are clustered into data sets based on the continuity between the laser readings. In the implementation, two laser readings with distance larger than $60 \mathrm{~mm}$ are regarded to be in different data sets, otherwise they are in the same data set. 
The data set that has too few or too many laser readings are discarded since they are not possible to be the candidates of a human leg. According to the experimental data, the relationship between the number of laser readings of a human leg and the distance from the robot is listed in Table 4.3.

Table 4.3. Relationship between the laser scan point numbers and the distance

\begin{tabular}{|c|c|}
\hline $\begin{array}{c}\text { Number of laser readings } \\
\text { (approximately) }\end{array}$ & $\begin{array}{c}\text { Distance between the robot and the person } \\
\text { (approximately) }\end{array}$ \\
\hline 15 & $1 \mathrm{~m}$ \\
\hline 12 & $1.2 \mathrm{~m}$ \\
\hline 10 & $1.5 \mathrm{~m}$ \\
\hline 8 & $1.8 \mathrm{~m}$ \\
\hline 6 & $2 \mathrm{~m}$ \\
\hline
\end{tabular}

In the human tracking application, the robot and the person are around $1.5 \mathrm{~m}$ away from each other, so according to Table 4.3, the data set that has less than 5 laser readings or more than 40 laser readings is discarded. In other words, a leg candidate data set must satisfy

$$
5 \leq \text { laserPoints } \leq 40
$$

\subsubsection{Leg Candidate Detection}

As introduced in section 3.4.2, if the minimum of the data set is not on the edges of the data set, this set is then further tested by diameter verification. Generally, a human leg has a diameter of $0.1 \mathrm{~m}$ to $0.25 \mathrm{~m}$, which means the distance between the two end points of a leg data set should be within this range. With the consideration that it is possible that one leg may be occluded by the other and the actual material of the pants may change the shape of the human leg in the laser frame, in our implementation, the 
lower bound and the upper bound of the width of a leg data cluster are extended to $80 \mathrm{~mm}$ and $300 \mathrm{~mm}$. For example, let $S=\left\{p_{k}, p_{k+1}, \ldots, p_{k+m}\right\}$ be a data set having $m$ laser readings (Figure 4.8), which satisfies equation (4-4) and equation (3-8), and $p_{k}$ and $p_{k+m}$ are the two end points of the data set, then the width $W$ of a leg data set should lie in

$$
80 \mathrm{~mm}<W<300 \mathrm{~mm}
$$

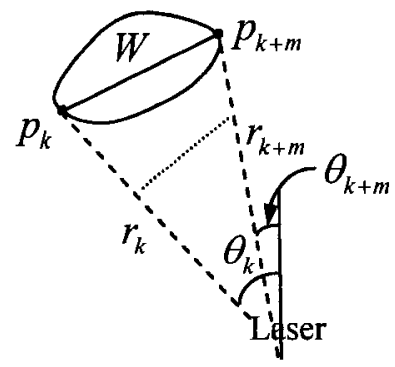

Figure 4.8. Width of the data set

The width $W$ of the set is calculated using the triangle equation. In Figure 4.8, the distance and angle data of laser readings $p_{k}$ and $p_{k+m}$ are denoted as $\left(r_{k}, \theta_{k}\right)$ and $\left(r_{k+m}, \theta_{k+m}\right)$, then

$$
W=\sqrt{r_{k+m}^{2}+r_{k}^{2}-2 r_{k+m} r_{k} \cos \left(\theta_{k+m}-\theta_{k}\right)}
$$

The difference between the locations of the person in two consecutive time steps should not be too large. The usual walking speed of a person is around $1.5 \mathrm{~m} / \mathrm{s}$ and the time step is $120 \mathrm{~ms}$ so the maximum distance a person can walk between two time steps is usually less than $200 \mathrm{~mm}$. Considering the ego-motion of the robot, the maximum distance is extended to be $400 \mathrm{~mm}$. Specifically,

$$
\operatorname{leg}_{\text {essp }}-400 m m<\operatorname{leg}_{c}<l e g_{\text {estp }}+400 m m
$$


where $l e g_{c}$ is the distance between the person's leg and the robot in the current time step, $\operatorname{leg}_{\text {essp }}$ is the distance between the person's leg and the robot estimated by the particle filter in the previous time step.

\subsubsection{Human Candidate Generation}

The location of each leg is approximated by the midpoint of the leg data set. Two leg candidates with distance less than $500 \mathrm{~mm}$ are grouped together. And the location of the person is approximated by the midpoint of the two legs. If one leg candidate is $500 \mathrm{~mm}$ away from other leg candidates, this leg candidate will be assigned to a person. In this case, the location of the person is approximated by the midpoint of the leg data set.

\subsubsection{Leg Detection Failure}

A counter is set in the program to count the times of tracking failure. If the leg detection fails continuously, the number in the counter is increased. If the number is larger than 8 , which means that the robot loses its target for about $1 \mathrm{~s}$, the robot will stop and the particles for particle filtering will be initialized again. If the number in the counter is less than 8 , the robot will move by following the commands from the previous time step. The counter is cleared as the human legs are detected again.

\subsubsection{Pseudo-Code}

The pseudo-code of the human detection module is given in Table 4.4, where the variable DetectionFailure is the name of the detection failure counter. 
Table 4.4. The human detection algorithm

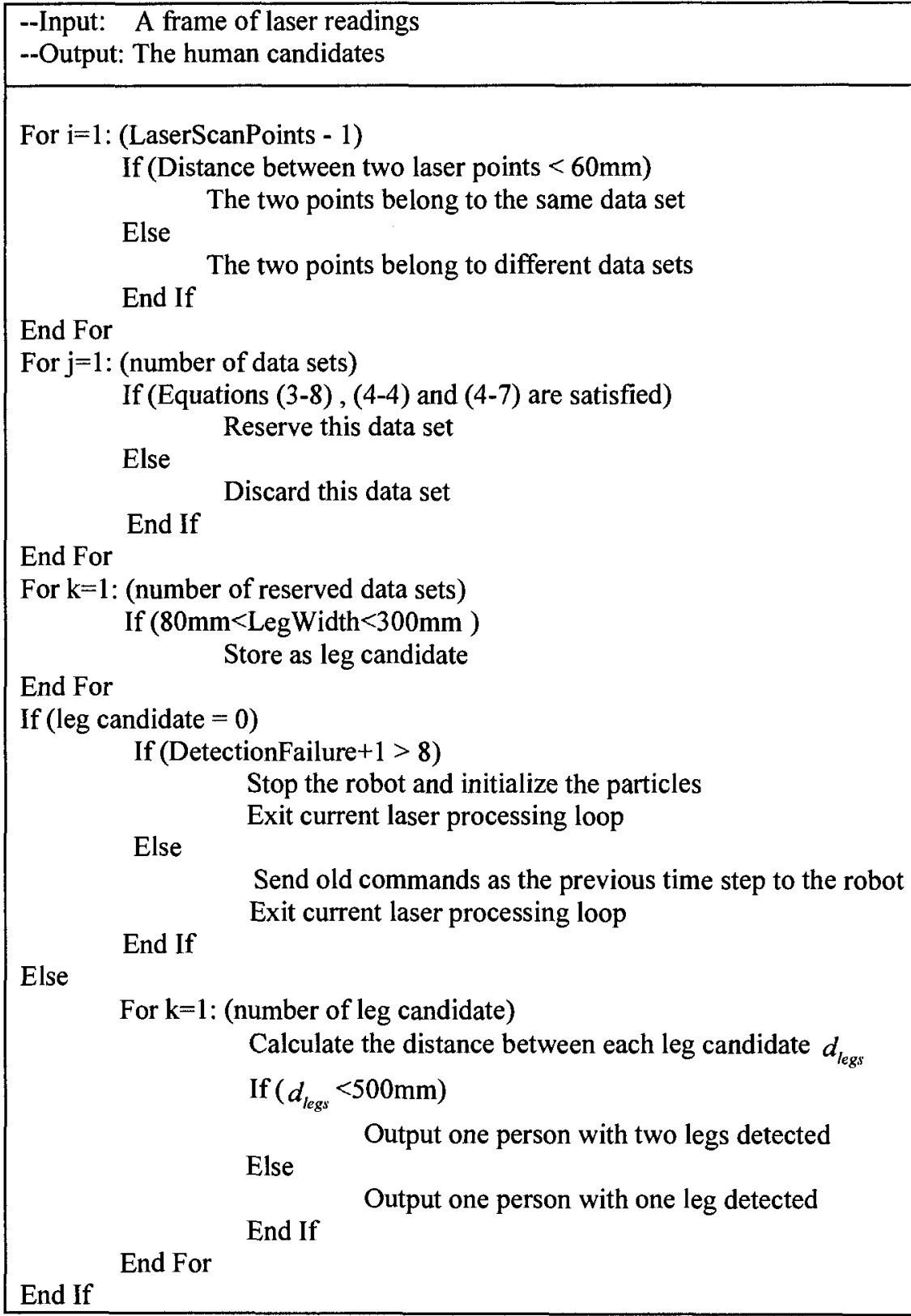

\subsection{Target Person Verification}

The module of target person verification is used to compare each human candidate with the target person model, in order to further verify whether a person is the target. 


\subsubsection{Rectangle Representation}

The target person is identified from the human candidates by image verification. Based on the location of the human candidates in the laser frame, the center and size of the rectangle of each human candidate in the image frame are determined.

Figure 4.9 and Figure 4.10 give examples for the target person verification. Figure 4.9 is the one-person case while Figure 4.10 is the two-person case.

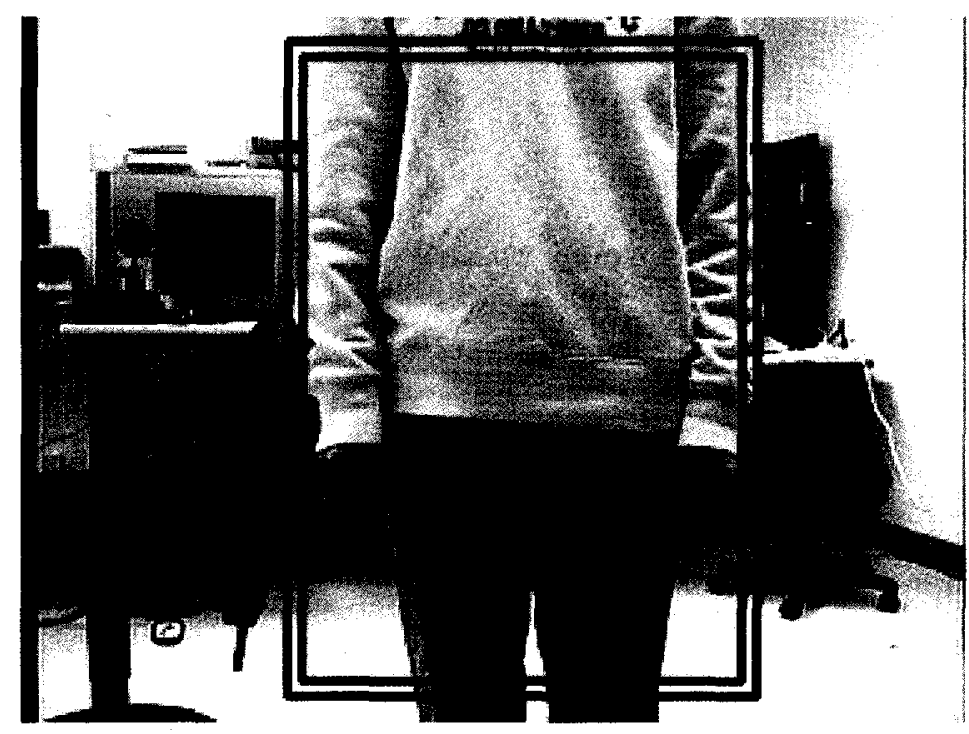

(a) The image frame

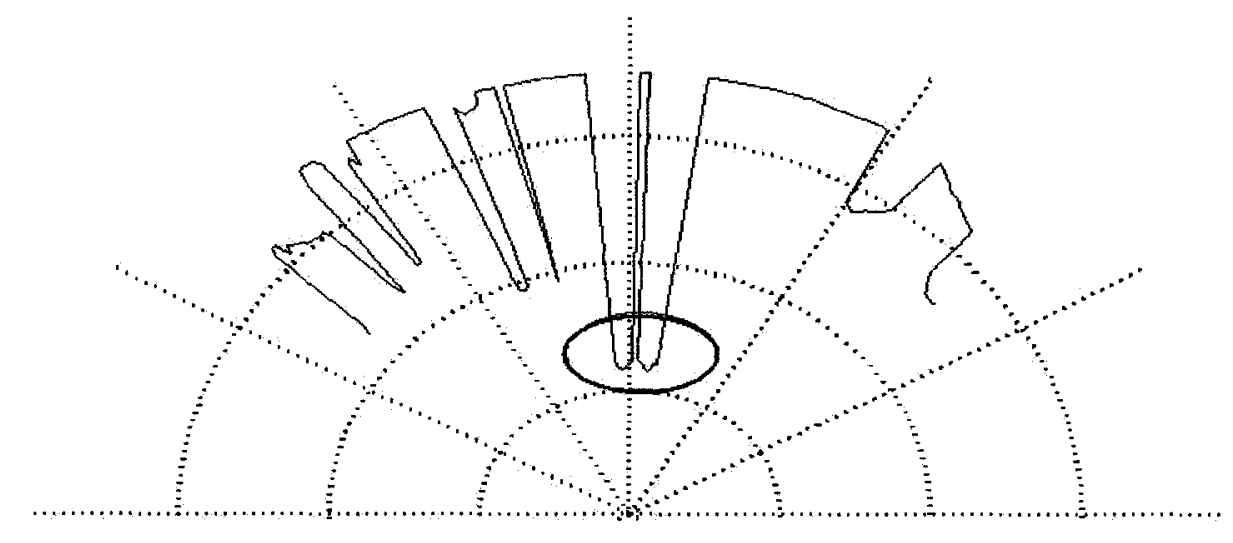

(b) The laser frame

Figure 4.9. Target person verification for one-person case 
Figure 4.9 (a) is the image frame and Figure 4.9 (b) is the corresponding laser frame. Based on the position of the detected legs in the laser frame, which is highlighted by a red ellipse in Figure 4.9 (b), the rectangle in Figure 4.9 (a) is determined. In Figure 4.9 (a), the blue rectangle represents the human candidate detected from the laser frame and the red rectangle represents the target after matching with the target model. In the one-person case, the human candidate is right the target, so the two rectangles are the same. For better visualization, the red rectangle is set to be smaller than the blue one.

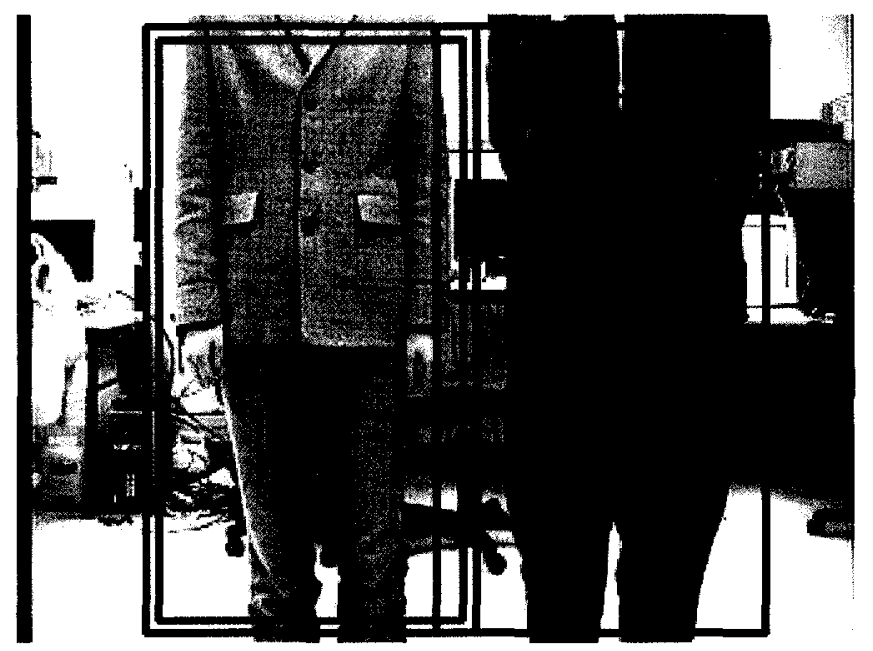

(a) The image frame

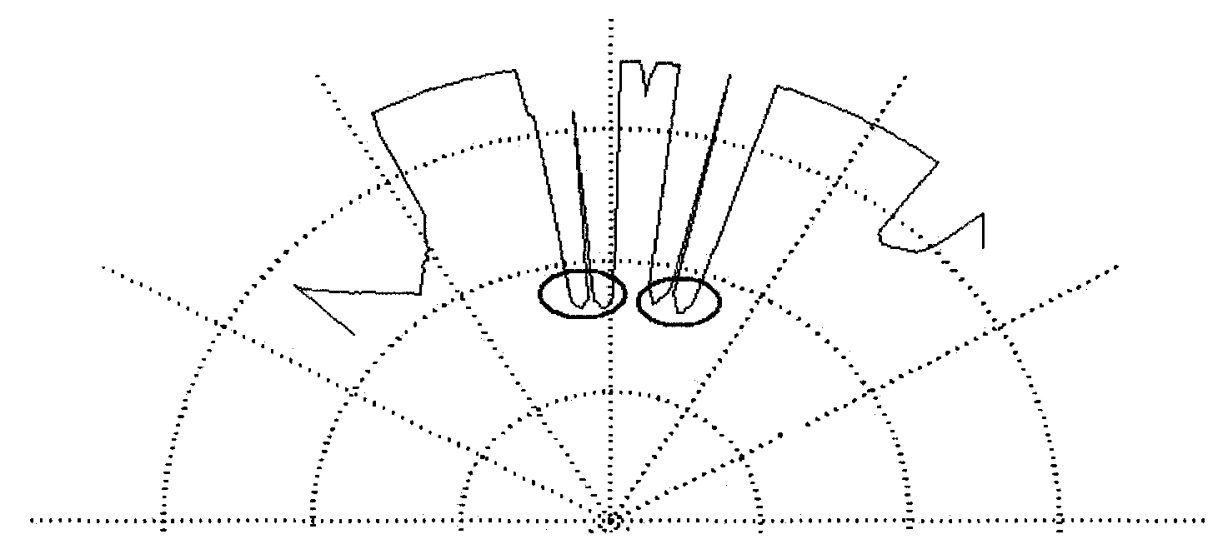

(b) The laser frame

Figure 4.10. Target person verification for two-person case 
In Figure 4.10 (a) there are two persons in the image. The legs of the two persons are highlighted with ellipses in Figure 4.10 (b). The red ellipse stands for the legs of the target person while the blue ellipse stands for the legs of the non-target person. The two blue rectangles in Figure 4.10 (a) show that there are two human candidates detected by the laser range finder and the red rectangle shows the target.

\subsubsection{Histograms}

The colour histogram and edge orientation histogram are calculated for each human candidate in the image frame (such as the blue rectangle image regions in Figure 4.9 (a) and Figure 4.10 (a)). The two histograms are calculated in the same way as introduced in section 4.2.1.2 and 4.2.1.3.

\subsubsection{Image Verification Failure}

As mentioned in Chapter 3, the view angle of the camera is smaller than the scan angle of the laser range finder. In cases that all the human candidates are outside the field of view of the camera, the image verification will fail. The detection failure counter acts in the same way as described in section 4.3.4.

\subsubsection{Pseudo-Code}

The pseudo-code for the target person verification module is shown in Table 4.5. 
Table 4.5. Pseudo-code of the target person verification module

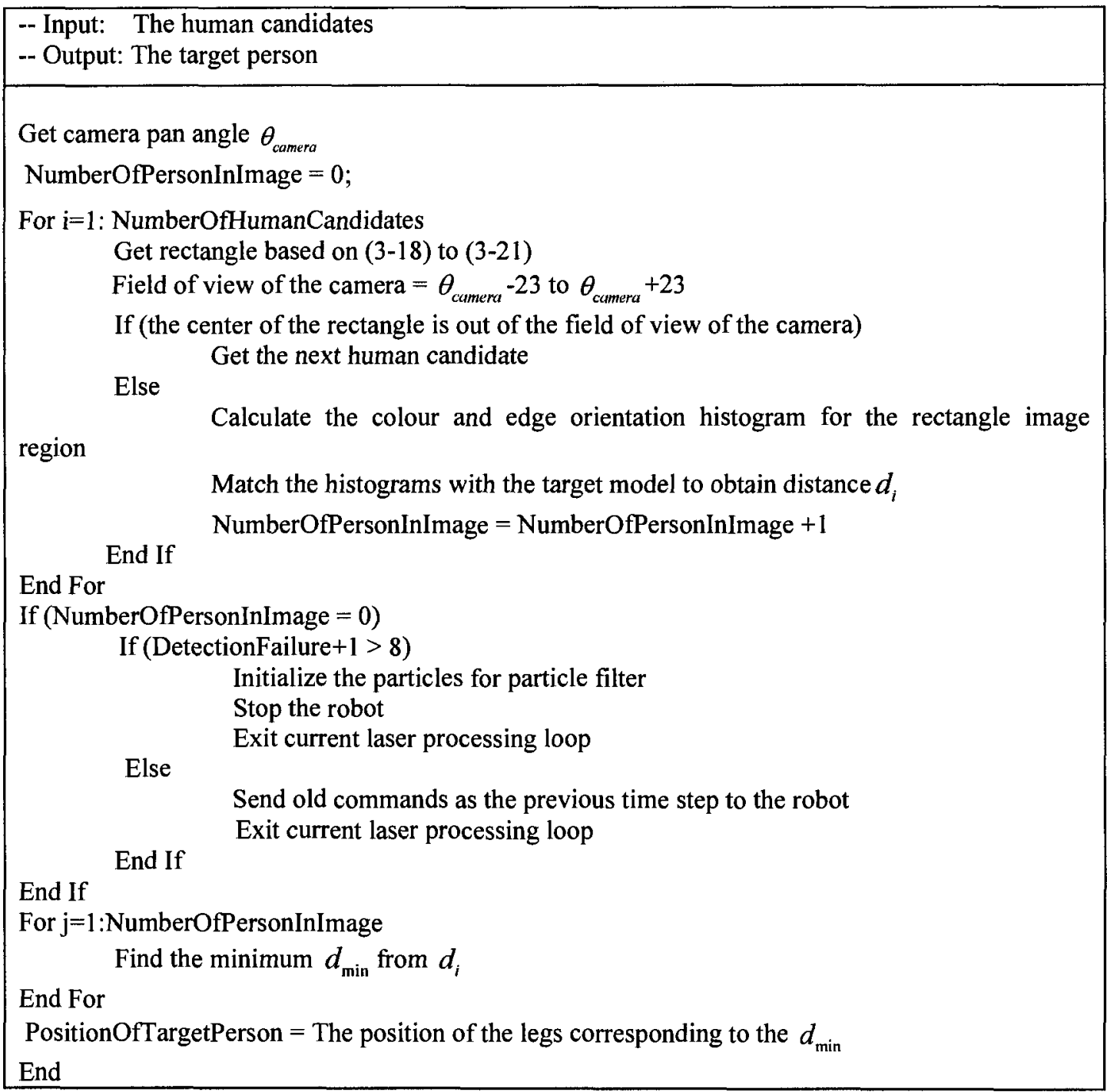

\subsection{State Estimation}

The maximum safety speed of the robot is $800 \mathrm{~mm} / \mathrm{s}$ and it is set to be $750 \mathrm{~mm} / \mathrm{s}$ for safety in the implementation, which requires that the average walking speed of the target person is less than $750 \mathrm{~mm} / \mathrm{s}$. So within one time step, the average distance the person is supposed to travel is $750 \mathrm{~mm} / \mathrm{s} \times 0.12 \mathrm{~s}=90 \mathrm{~mm}$. Based on this assumption and the experimental results (in Chapter 5), the standard deviations of the two velocity 
components in Equation (3-30) are chosen as $\sigma_{v_{x}}=\sigma_{v_{y}}=1 \mathrm{~m} / \mathrm{s}$. The number of particles for the particle filtering is set to be 150 .

The variance for distance $\sigma_{d}$ and variance for angle $\sigma_{A}$ in Equation (3-36) are used for calculating the weight of each particle. As we know, the weight is proportional to the likelihood probability. So the two variances should ensure that the two likelihoods are less than 1. In the implementation, the two parameters are set to be $\sigma_{d}=100$ and $\sigma_{A}=5$

Suppose the target person is at $\left(x_{\text {model }}, y_{\text {model }}\right)$ when the target person model is constructed. Then each particle, which is a state vector $\left(x^{i}, y^{i}, v_{x}^{i}, v_{y}^{i}\right)$, is initialized as

$$
\left(x^{i}, y^{i}\right)=\left(x_{\text {model }}, y_{\text {model }}\right)
$$

and

$$
\left(v_{x}^{i}, v_{y}^{i}\right)=(0,0)
$$

As introduced in section 3.6.1, the purpose of re-sampling is to resolve the problem of degeneracy. In the implementation, the particles are sorted from high weights to low weights before re-sampling. The particles are duplicated by multiplying their weights with the total number of particles in the re-sampling step.

The pseudo-code of particle filtering is shown in Table 4.6, where the symbol $N$ represents the number of particles. 
Table 4.6. Pseudo-code of particle filtering

-- Input: Position of the target person measured by the laser range finder

-- Output: Estimated position and velocity of the target person

Initialization: Done in the first time step

For $\mathrm{i}=1: N$

Initialize particle $\mathbf{x}_{0}^{i}=\left(x_{0}^{i}, y_{0}^{i}, v_{x 0}^{i}, v_{y 0}^{i}\right)$ with Equations (4-8) and (4-9)

Set the weight $w_{0}^{\prime}=1 / N$

End

Propagation, weight computation, state estimation, re-sampling: Done in the following time steps

For $\mathrm{i}=1: N$

Perform ego-motion compensation based on Equation (3-31).

Propagate $\mathbf{x}_{k}^{i}$ to $\mathbf{x}_{k+1}^{i}$ according to Equation (3-30).

\section{End For}

For $\mathrm{i}=1: N$

Calculate $w_{k+1}^{i}$ using Equation (3-36).

End For

For $\mathrm{i}=1: N$

$$
\text { Normalize } w_{k+1}^{i}: w_{k+1}^{i}=\frac{w_{k+1}^{i}}{\sum_{i=0}^{N-1} w_{k+1}^{i}}
$$

End For

Calculate the estimation state $\hat{\mathbf{x}}_{k+1}=\sum_{i=0}^{N-1} w_{k+1}^{i} \mathbf{x}_{k+1}^{i}$.

Sort the particles from high weight to low weight.

$k=0$

For $\mathrm{i}=1: N$

$$
\mathrm{n}=w_{k+1}^{i} N
$$

For $\mathrm{j}=\mathbf{l}: \mathbf{n}$

Generate a new particle with state $\mathbf{x}_{k+1}^{i}$.

$$
\begin{gathered}
k=k+1 \\
\text { If }(k=N)
\end{gathered}
$$

End If

End For

End For

For $\mathrm{i}=1: N$

$$
w_{k+1}^{i}=1 / N .
$$

End 


\subsection{Control Commands Generation}

According to the estimated location and velocity of the target person by the particle filtering, heading and velocity commands are generated and sent to the robot controller one step in advance.

To keep the person in the center of the field of view of the robot, the heading angle of the robot should be equal to the estimated angle between the person and the robot. However when the target has only slight movement on his or her legs, it is found that it will make the person more comfortable if the robot keeps still until the person moves apparently. Based on this observation, the robot is set to turn only when the angle between the robot and the person is beyond $\left[-6^{\circ},+6^{\circ}\right]$.

When the heading and translation commands are sent to the robot, the robot may not be able to finish the desired turning or translation within one time step because of the relatively large response time of the mechanical equipment. For example, the heading command sent to the robot in current time step is $9.56^{\circ}$, in the next time step, the angle the robot has turned, which is obtained from odometer readings, is $2.58^{\circ}$. Although the odometer readings contain errors, we can still get an idea about the turning capability of the robot. As a consequence, the target person may walk out of the view of the camera. In order to compensate for the slow heading movement of the robot, the pan angle of the camera is set to be

$$
\text { panAngle = heading }
$$

where heading is the turning angle of the robot generated by the particle filter (see Equation (3-39)). 
The translation velocity is set for the robot in each time step based on the velocity of the target person estimated by the particle filter with a distance control. Specifically, when the distance between the robot and the person is less than $1.2 \mathrm{~m}$, the translation velocity of the robot is set to be 0 , that is, no translation command is sent to the robot. When the distance is larger than $1.2 \mathrm{~m}$ and less than $1.5 \mathrm{~m}$, the translation velocity of the robot is set to be the velocity of the person. If the distance is larger than $1.5 \mathrm{~m}$, the robot is instructed to move with the maximum velocity, which is $750 \mathrm{~mm} / \mathrm{s}$.

\subsection{Requirements of the System}

From the above discussions on the implementation details, the requirements for the human tracking mobile robot system are summarized as follows:

1. In the target model construction stage, only the target person is allowed to move in the field of view of the robot. More objects moving in the scene will make the target model rectangle contain too much image information that does not belong to the target person, which will make the target person model less accurate. As a result, the target person verification module will be less effective.

2. For stable human tracking, the target person is expected to walk with an average speed lower than $750 \mathrm{~mm} / \mathrm{s}$.

3. If there are too many leg-like objects around the person, it is likely that there will be too many human candidates detected by the laser range finder. If this is the case, the computation complexity for calculating the histograms of the rectangle image region for each human candidate will be high. For example, if there are two persons in the scene, the time for performing image processing for the two rectangle image regions is close to $120 \mathrm{~ms}$. If there are even more persons in the 
scene, the time for image processing tasks will exceed $120 \mathrm{~ms}$, which will prolong the cycle for particle filtering. Therefore the estimation accuracy will be degraded. 


\section{Chapter 5 Performance and Results}

The performance of the proposed method introduced in chapter 3 and chapter 4 is evaluated in this chapter.

\subsection{Experiment Platform}

The experiments are performed on the Performance PeopleBot ${ }^{\mathrm{TM}}$ robot in the lab environment.

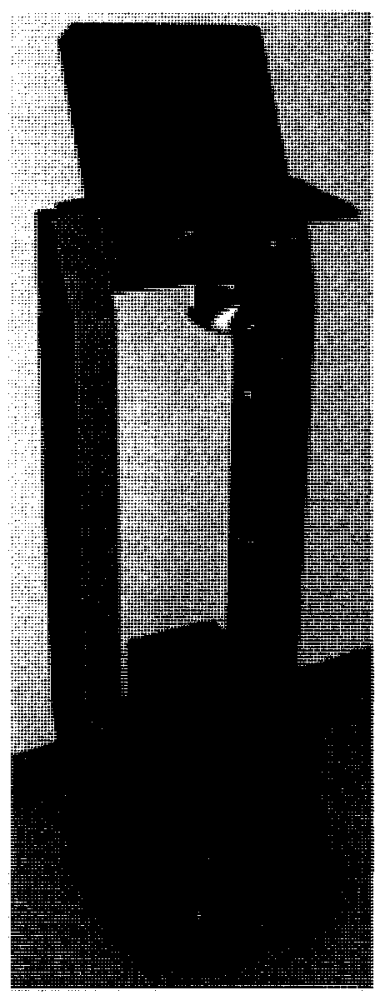

(a)

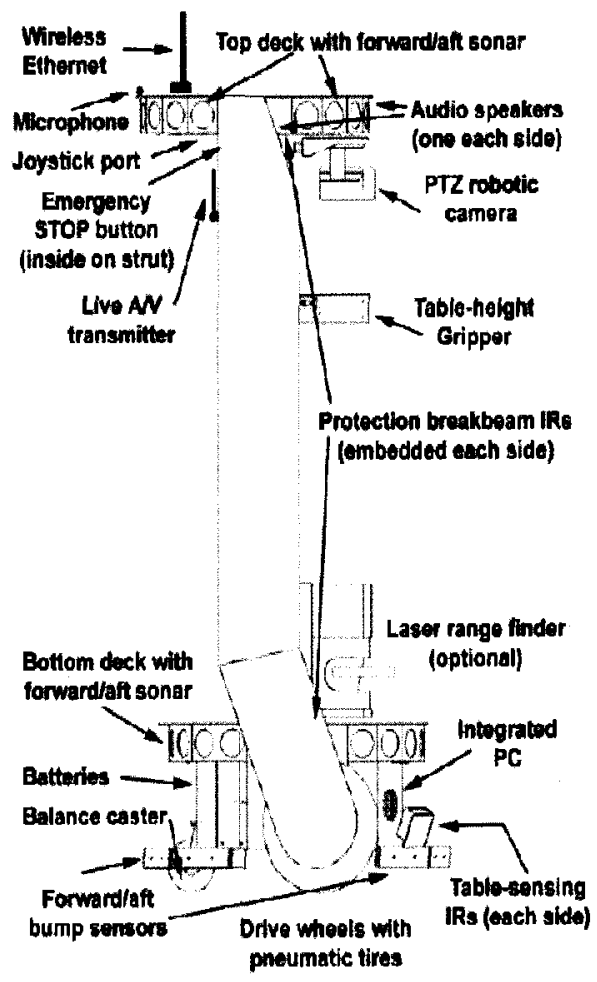

(b)

Figure 5.1. PeopleBot ${ }^{\mathrm{TM}}$ features and accessories [57]

Figure 5.1 (a) is a picture of the robot. The accessories mounted on the robot are shown in Figure 5.1 (b) (adopted from the manual [57]). The Performance PeopleBot ${ }^{\mathrm{TM}}$ 
robot is designed for human-robot interaction applications. The height of the robot is $115 \mathrm{~cm}$, which is compatible to the height of an adult people. This feature makes it easier for a human to communicate with the robot.

\subsection{Experiments}

The experiments test the performance of the developed human tracking mobile robot system. All the experiments took place in the indoor environment.

\subsubsection{Comparison of Leg Detection Algorithms}

The leg detection algorithm based on the arc assumption [29] and the one developed in this thesis in section 3.4.2 are compared. In the experiment, the robot kept still and only the camera moved. A person was walking along an arc, which was centered at the robot with radius $1.2 \mathrm{~m}$. For both algorithms, the person was moving along a similar trajectory and at a similar velocity. The tracking process lasted for 1000 cycles, which is 2 minutes. The results are shown in Table 5.1.

Table 5.1. The leg detection algorithms comparison

\begin{tabular}{|c|c|c|}
\hline Items & Arc assumption & Developed algorith m \\
\hline One leg detected & $48.8 \%$ & $20.2 \%$ \\
\hline Two legs detected & $31.8 \%$ & $78.4 \%$ \\
\hline Undetected & $19.4 \%$ & $1.4 \%$ \\
\hline
\end{tabular}

Table 5.1 shows that the algorithm developed in this thesis has satisfactory undetected probability. 


\subsubsection{Tracking Performance of the Particle Filter}

In this experiment, the robot was at rest but the camera was allowed to pan. The target person was moving in the field of view of the robot. This experiment is performed to evaluate the tracking performance of the particle filter.

The performance of the particle filter depends on the values of its parameters, which are the particle numbers and the standard deviations of the random Gaussian distribution $\sigma_{v_{x}}$ and $\sigma_{v_{y}}$.

Fist, we must choose appropriate standard deviations. The standard deviation of a random distribution determines how widely spread the samples are from the expectation. Most of the samples will be in the vicinity of the expectation if the standard deviation is too small. If this is the case, the particle filter cannot estimate the state of the person correctly. On the other hand, the samples will be widely spread and the accuracy of the estimation will be low if the standard deviation is too large.

A person was walking in front of the robot. A rope was tied to the person's two legs to ensure the distance between the two legs, which is the step, to be $300 \mathrm{~mm}$. The person was listening to a clock to adjust his or her pace to be two steps per second. Therefore, the walking speed of the person was set to be $600 \mathrm{~mm} / \mathrm{s}$. This velocity is regarded as the real velocity of the person. The tracking procedure lasted for 72 seconds. During tracking, the particle filter estimated the velocity for the walking person.

Figure 5.2 shows each estimated velocity under different standard deviations, which are $\sigma_{v_{x}}=\sigma_{v_{y}}=0.2, \sigma_{v_{x}}=\sigma_{v_{y}}=1, \sigma_{v_{x}}=\sigma_{v_{y}}=1.5$, and $\sigma_{v_{x}}=\sigma_{v_{y}}=2$. This figure illustrates how the estimated velocity varied during the tracking. 


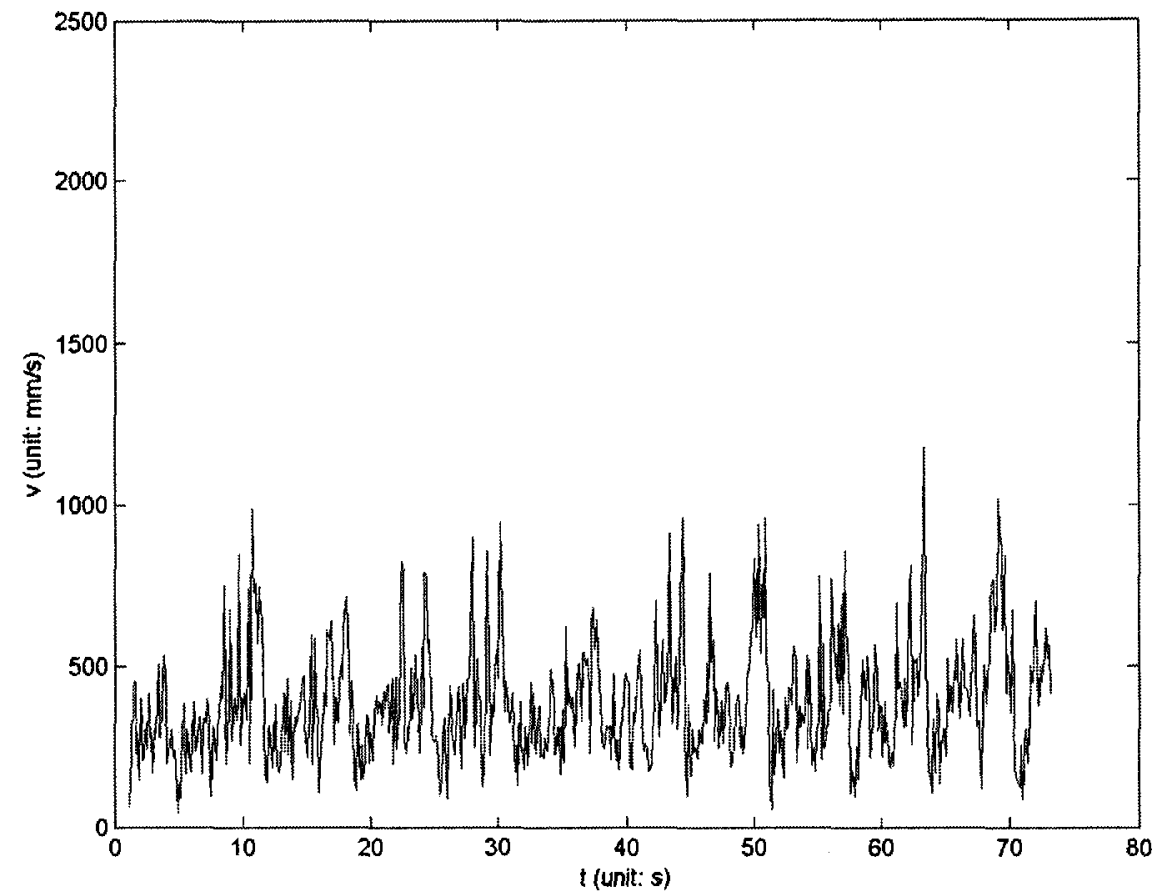

(a) $\sigma_{v_{x}}=\sigma_{v_{y}}=0.2$

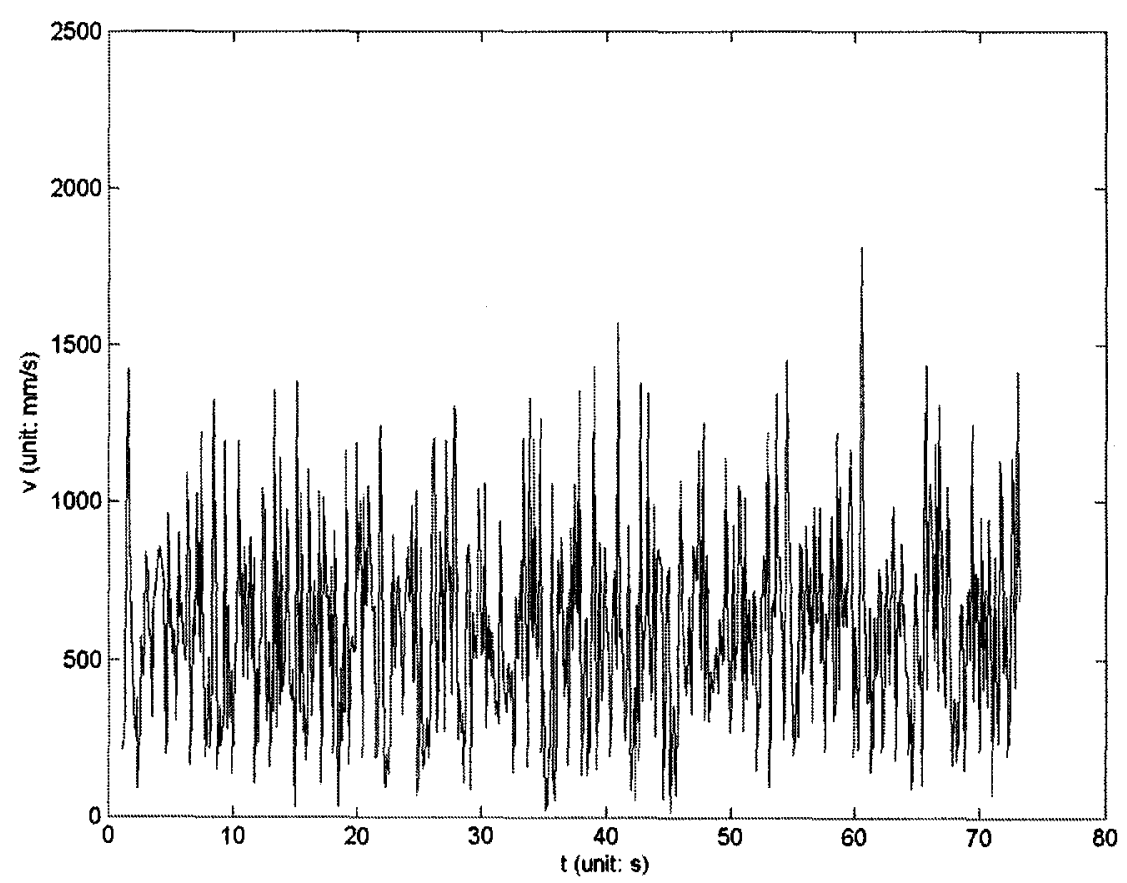

(b) $\sigma_{v_{x}}=\sigma_{v_{y}}=1$ 


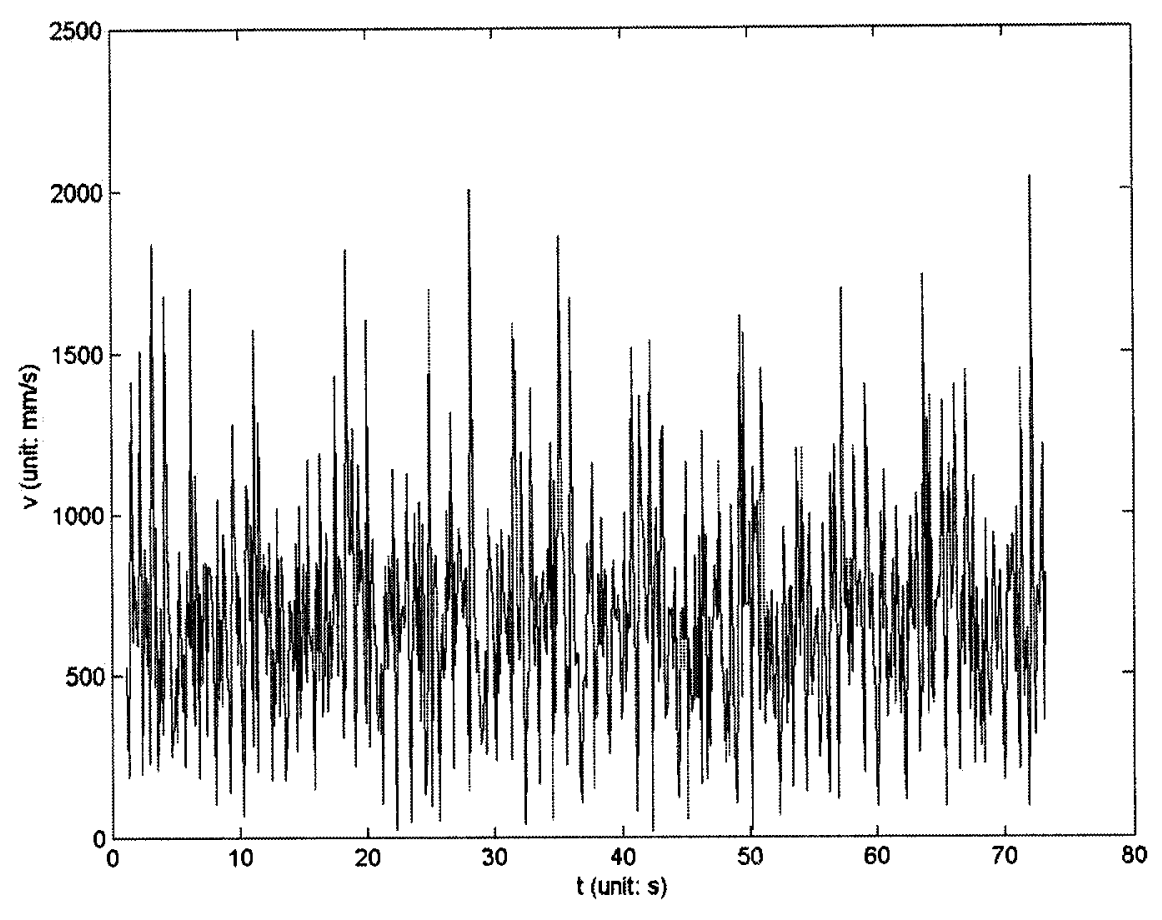

(c) $\sigma_{v_{x}}=\sigma_{v_{y}}=1.5$

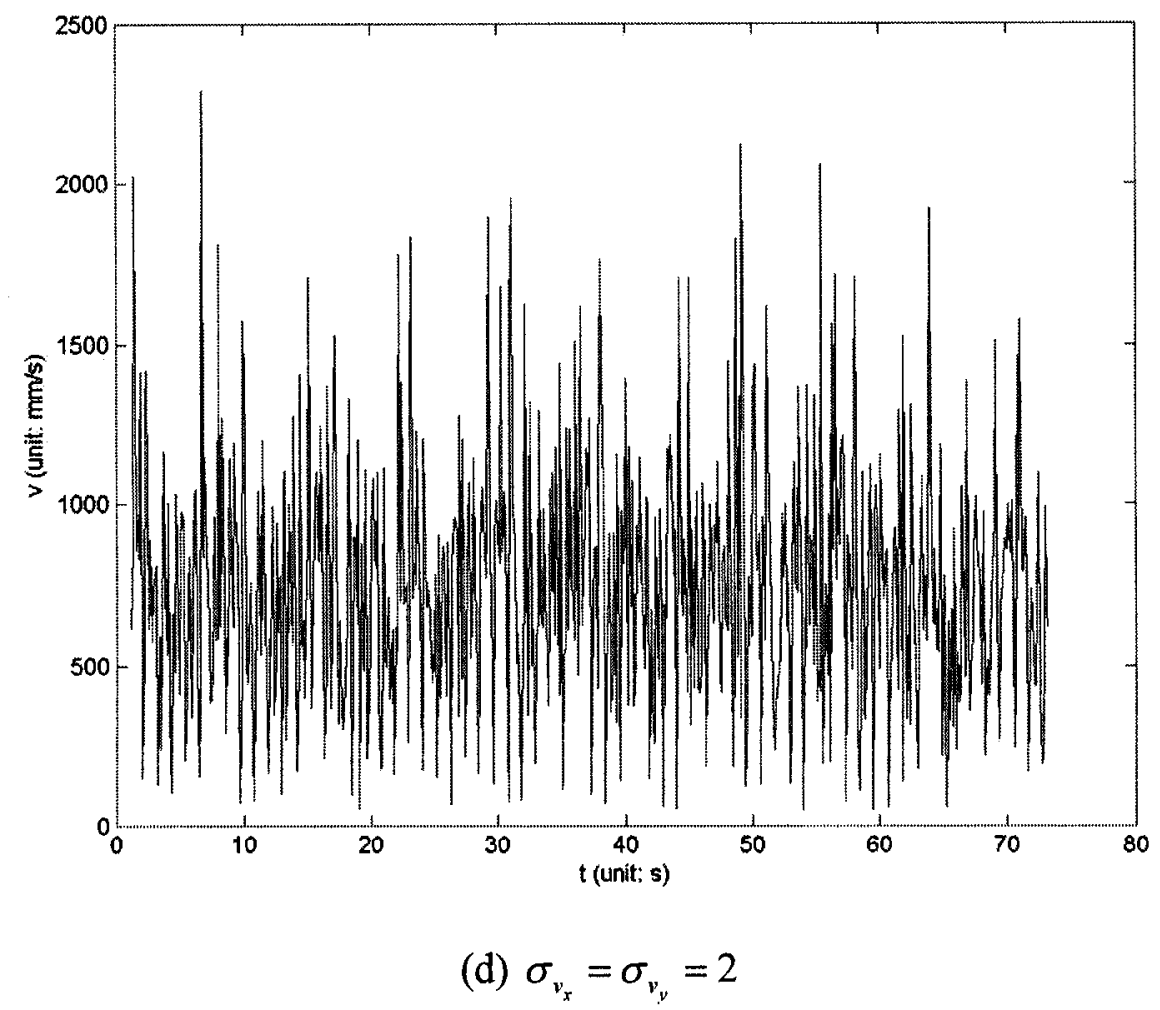

Figure 5.2. Estimated velocities of different standard deviations $\sigma_{v_{x}}$ and $\sigma_{v_{y}}$ 
Table 5.2 gives the average and standard deviation of each estimated velocity under different $\sigma_{v_{x}}$ and $\sigma_{v_{y}}$, which uses the same data as in Figure 5.2.

Table 5.2. Average and standard deviation of the estimated velocity

\begin{tabular}{|c|c|c|c|c|}
\hline & $\sigma_{v_{x}}=\sigma_{v_{y}}=0.2$ & $\sigma_{v_{x}}=\sigma_{v_{y}}=1$ & $\sigma_{v_{x}}=\sigma_{v_{y}}=1.5$ & $\sigma_{v_{x}}=\sigma_{v_{y}}=2$ \\
\hline $\begin{array}{c}\text { Average } \\
(\mathrm{mm} / \mathrm{s})\end{array}$ & 375.9098 & 595.0645 & 674.1799 & 738.4279 \\
\hline $\begin{array}{c}\text { Standard deviation } \\
(\mathrm{mm} / \mathrm{s})\end{array}$ & 183.1590 & 305.4980 & 348.7118 & 385.5075 \\
\hline
\end{tabular}

In Table 5.2, when $\sigma_{v_{x}}$ and $\sigma_{v_{y}}$ are small $\left(\sigma_{v_{x}}=\sigma_{v_{y}}=0.2\right)$, the average estimated velocity $(375.9098 \mathrm{~mm} / \mathrm{s})$ is too small to reflect the real velocity $(600 \mathrm{~mm} / \mathrm{s})$. The robot cannot be able to follow the person and finally it will loose the target. When $\sigma_{v_{x}}=\sigma_{v_{y}}=1$, the average of the estimated velocity $(595.0645 \mathrm{~mm} / \mathrm{s})$ is close to the real velocity, which means the particle filter can estimate the state of the person correctly. The average estimated velocity when $\sigma_{v_{x}}=\sigma_{v_{y}}=1.5$, which is $674.1799 \mathrm{~mm} / \mathrm{s}$, can also reflect the real velocity of the walking person but the standard deviation of the estimated velocity $(384.7188 \mathrm{~mm} / \mathrm{s})$ is larger than that when $\sigma_{v_{x}}=\sigma_{v_{y}}=1(305.4980 \mathrm{~mm} / \mathrm{s})$. When $\sigma_{v_{x}}$ and $\sigma_{v_{y}}$ are larger $\left(\sigma_{v_{x}}=\sigma_{v_{y}}=2\right)$, the average and standard deviation of the estimated velocity are both larger which makes the particle filter less accurate. Therefore, $\sigma_{v_{x}}=\sigma_{v_{y}}=1$ is used in the following experiments.

Second, we must determine the particle numbers. Recall that in the discussions about the particle filter in Chapter 3, the more the particles the closer the estimation is to the true posterior distribution. On the other hand the more particles, the more processing 
time for the particle filtering procedure. So there is always a tradeoff between the computation cost and the tracking performance.

A person was walking in front of the robot with a walking speed less than $750 \mathrm{~mm} / \mathrm{s}$. The robot kept tracking the moving person for 1 minute. Figure 5.3 shows the trajectory of the person measured by the laser range finder, which is marked as '.-', and the trajectory estimated by the particle filter, which is marked as 't-', under different particle numbers. The average filtering time under different particle numbers is shown in Table 5.3. The filtering time is the time duration from getting laser readings to sending commands to the robot. The average filtering time is obtained by averaging the filtering time on 200 time steps. Figure 5.3 (a) (50 particles) shows that the estimated trajectory is a bit far from the measurement. Figure 5.3 (b) (100 particles) gives a better performance than (a). Figure 5.3 (c) (150 particles) and Figure 5.3 (d) (200 particles) both track the moving person well whereas (d) needs more computation time (see Table 5.3). With the consideration of the tradeoff between the computation cost and the tracking performance, 150 particles were chosen for the particle filtering.

Table 5.3. Average filtering time under different particle numbers

\begin{tabular}{|c|c|c|c|c|}
\hline & 50 particles & 100 particles & 150 particles & 200 particles \\
\hline $\begin{array}{c}\text { Average filtering time } \\
(\mathrm{ms})\end{array}$ & 56.7635 & 75.2122 & 85.4911 & 93.6210 \\
\hline
\end{tabular}




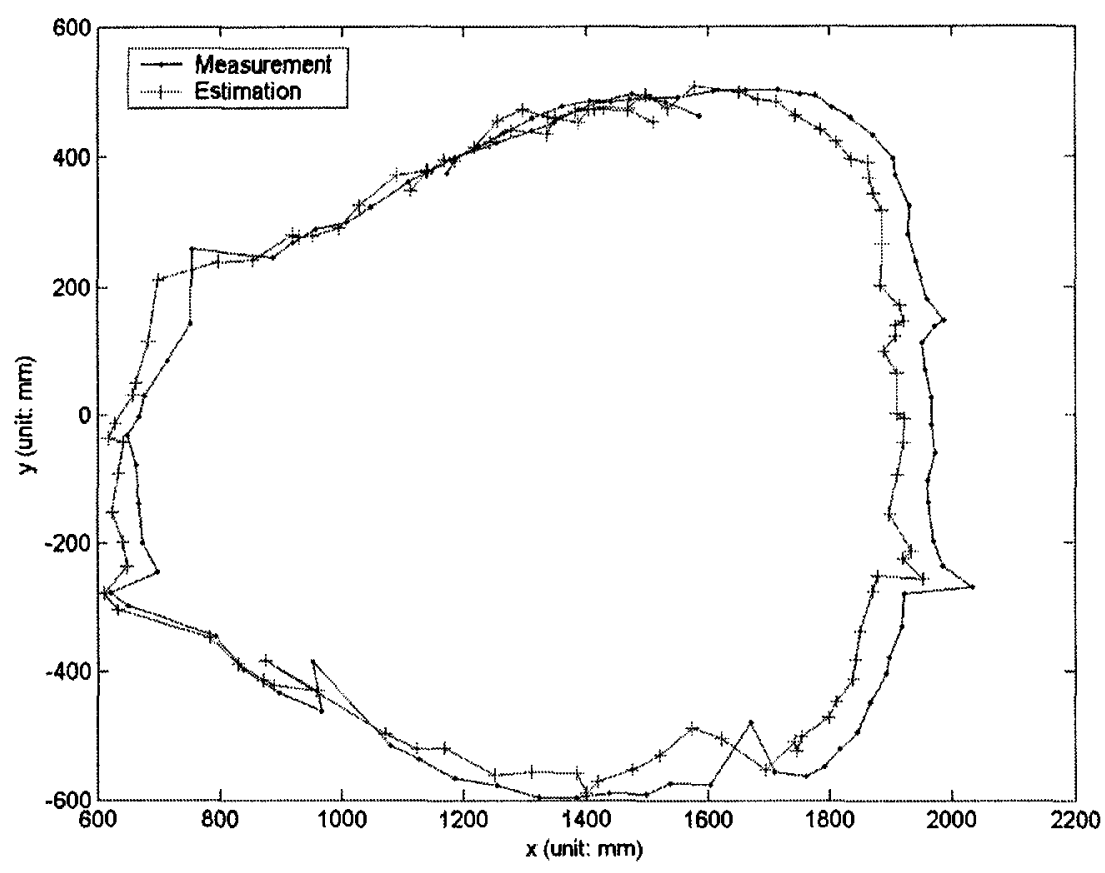

(a) Particle numbers: 50

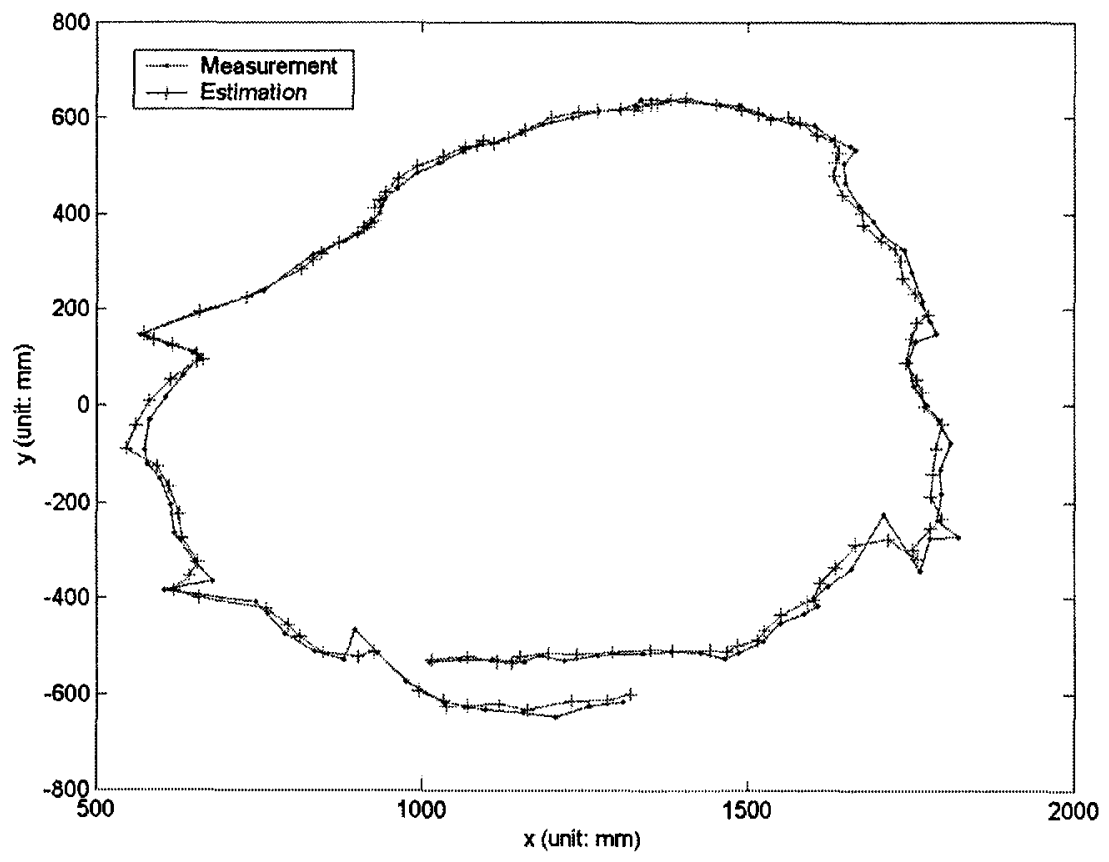

(b) Particle numbers: 100 


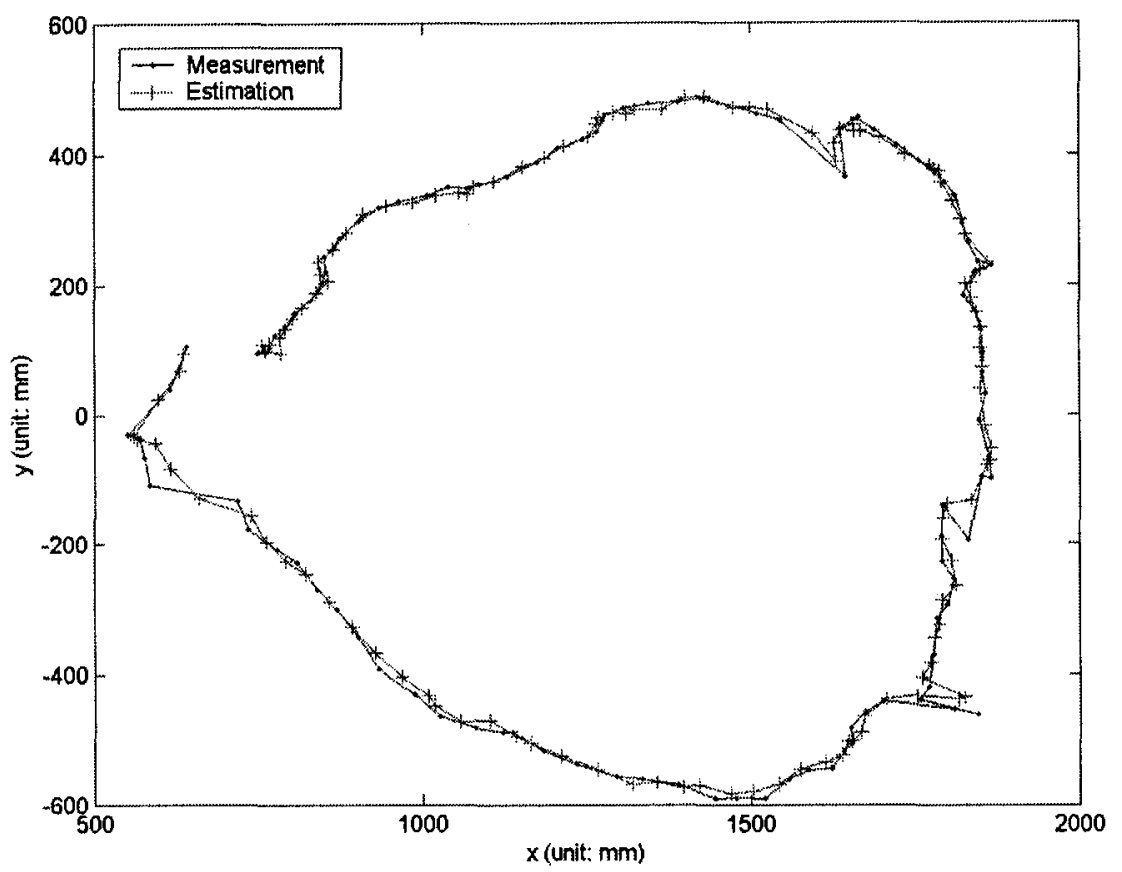

(c) Particle numbers: 150

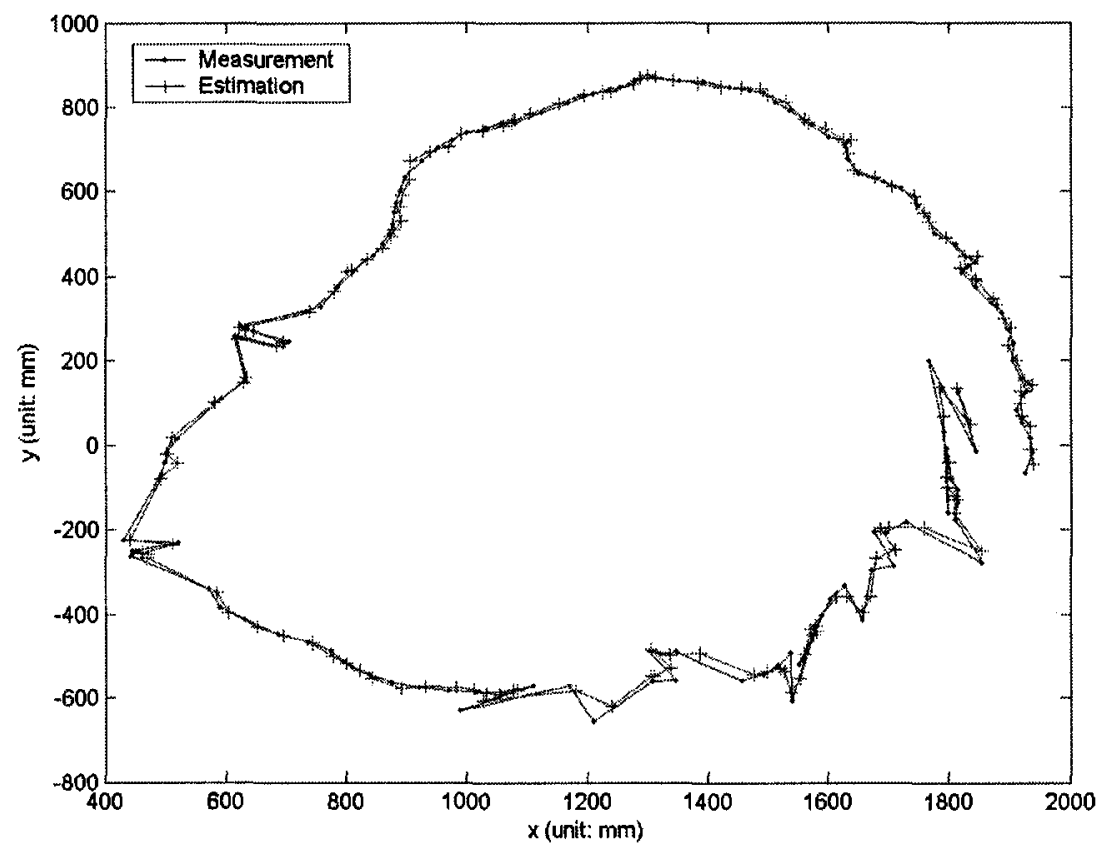

(d) Particle numbers: 200

Figure 5.3. Change of particle numbers 


\subsubsection{Following One Person}

In this section, the tracking results for the case that there is only one person (the target person) in the field view of the robot are given.

The trajectory of the target person is shown in Figure 5.4 (a). Figure 5.4 (b) is the segmented image used to build the target person model. The video frames are shown in Figure $5.4(\mathrm{c})$.

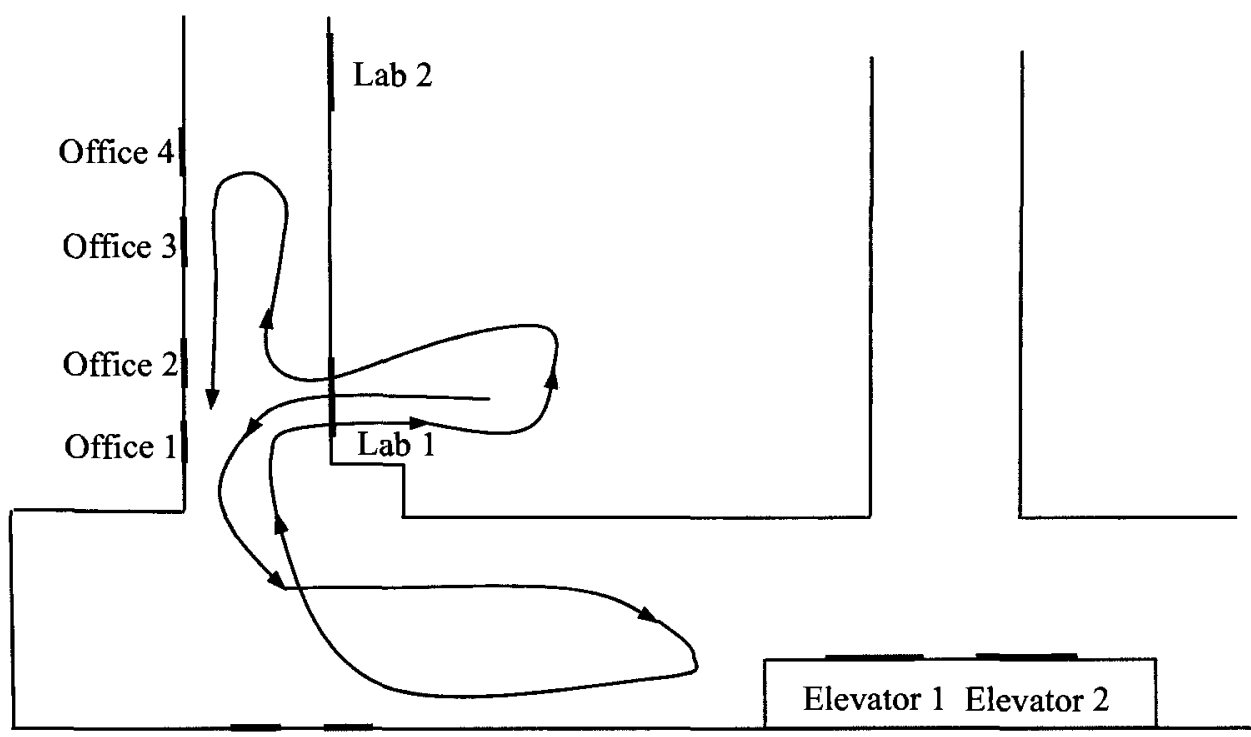

Stairs Washroom

(a) The trajectory of the walking person

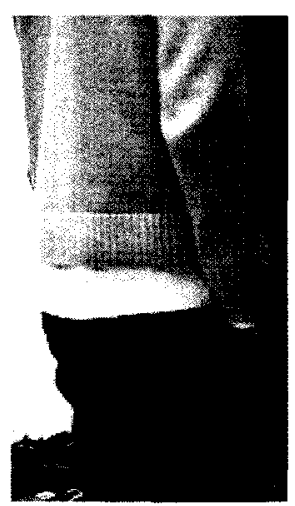

(b) Segmented image 


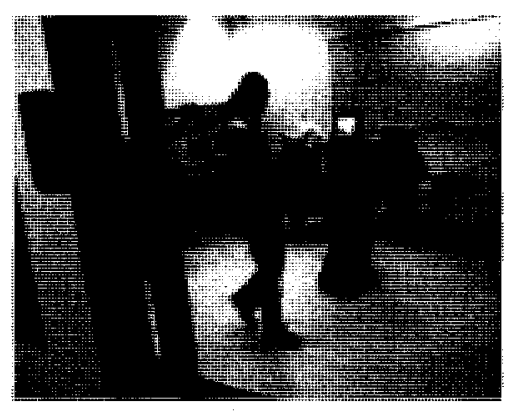

(c-1) Frame 8

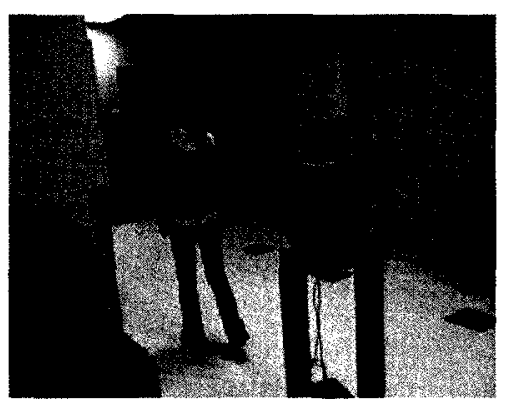

(c-4) Frame 466

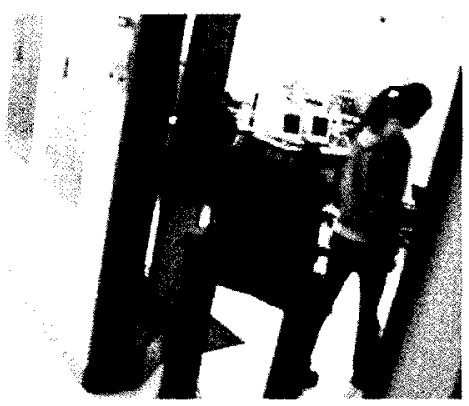

(c-7) Frame 1058

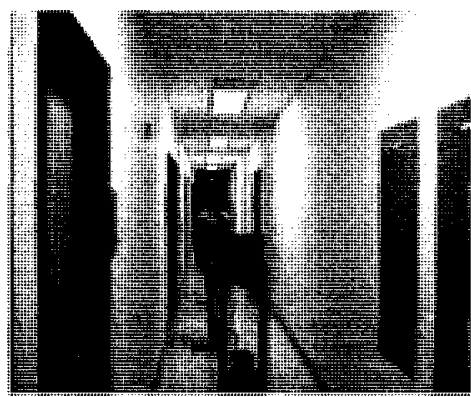

(c-10) Frame 1628

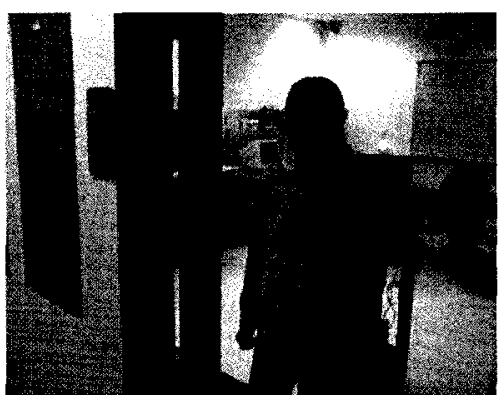

(c-2) Frame 155

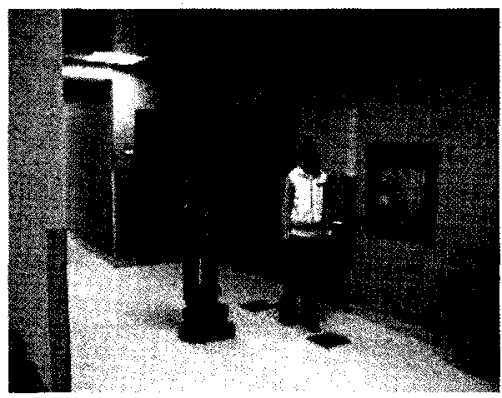

(c-5) Frame 619

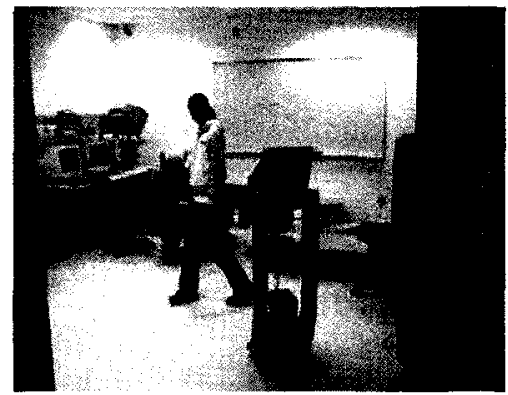

(c-8) Frame 1184

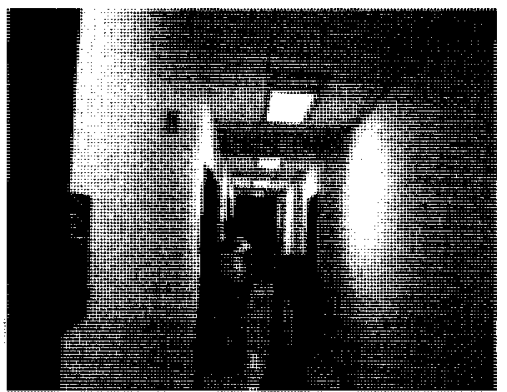

(c-11) Frame 1797

(c) Video frames

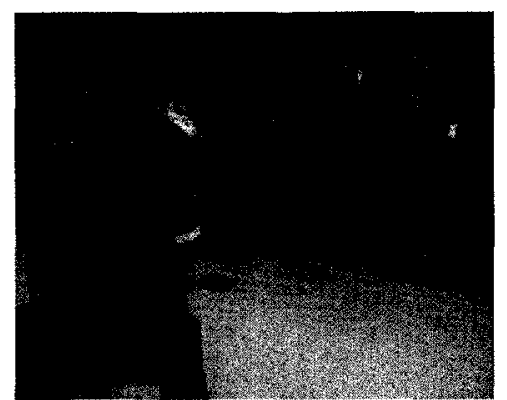

(c-3) Frames 400

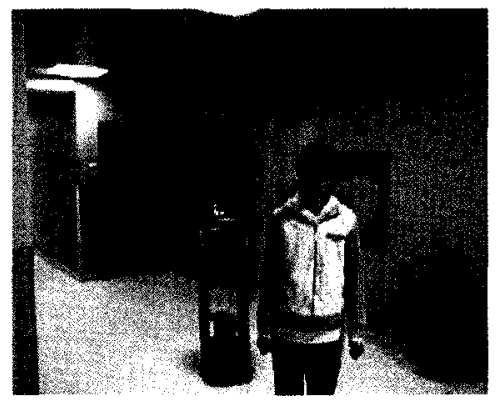

(c-6) Frames 728

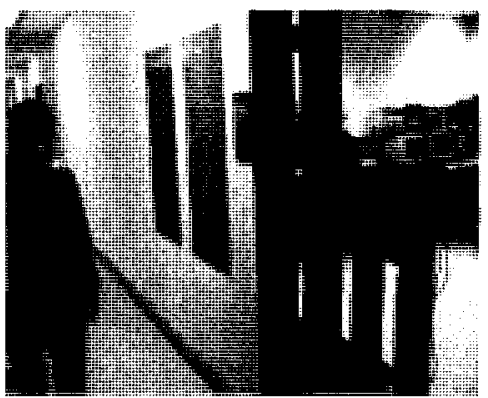

(c-9) Frame 1425

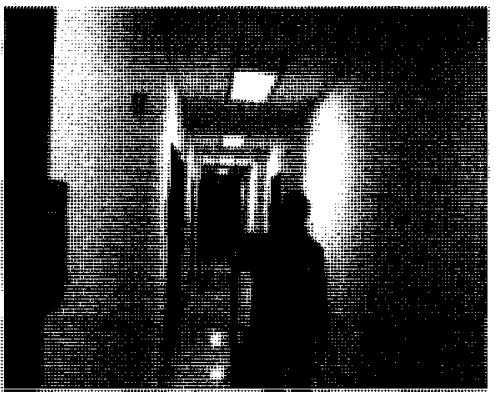

(c-12) Frame 1918

Figure 5.4. Following one person 
Figure 5.4 (c-1) to Figure 5.4 (c-3) show the person was walking from the lab into the corridor on her left hand. From Figure 5.4 (c-4) to Figure 5.4 (c-6), the person was turning in the corridor. Figure 5.4 (c-7) to Figure 5.4 (c-9) present that the robot was following the person back into the lab and out of the lab into the corridor on her right hand. Figure 5.4 (c-10) to Figure 5.4 (c-12) show that the robot kept following the person when the person was turning in the corridor.

\subsubsection{Target Person Recognition}

The tracking results in this section are shown for the case that there are two persons in the field of view of the robot, but only one is the target.

\subsubsection{The Experiment on Person 1}

The trajectories of the target person (person 1, solid line) and the non-target person (person 2, dashed line) are shown in Figure 5.5 (a). Figure 5.5 (b) is the segmented target person image. Figure 5.5 (c) are the image frames recorded by the onboard computer of the robot, which show the persons 'seen' by the camera. These frames were saved at the same time the video in Figure 5.5 (d) was recorded. In Figure 5.5 (c), the blue rectangles represent the human candidates detected by the laser range finder and the red rectangles represent the target person recognized by the image verification. Take a look at Figure 5.5 (c-6), the non-target person was bounded with a blue rectangle but the center of this rectangle drifted much to the left. The reason that the rectangle was not located on the center of the person is that the right leg of the person was not detected by the leg detection algorithm and the person was recognized by the one-leg pattern. Figure 5.5 (d) are the video frames. 


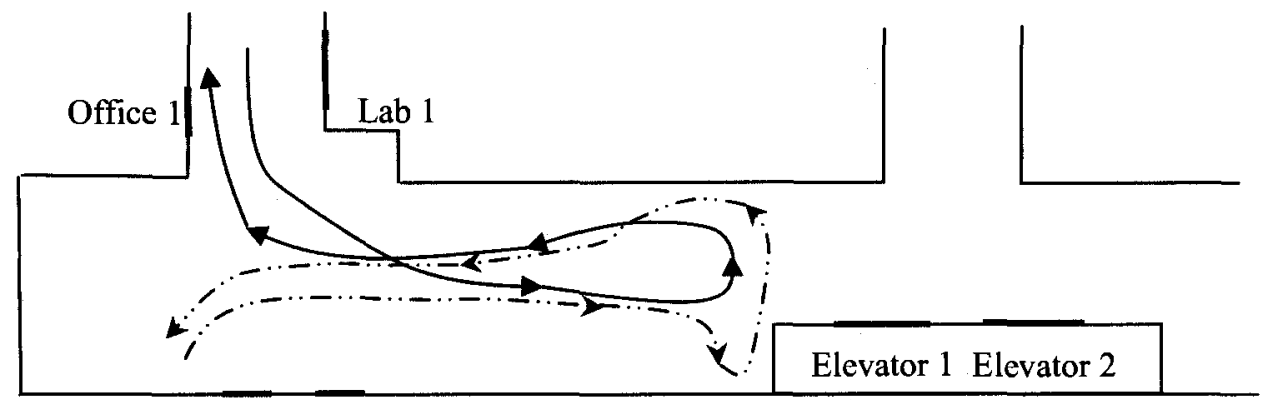

Stairs Washroom

(a) The trajectories of the target person and the non-target person

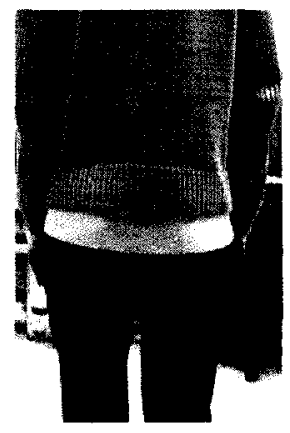

(b) Segmented target image
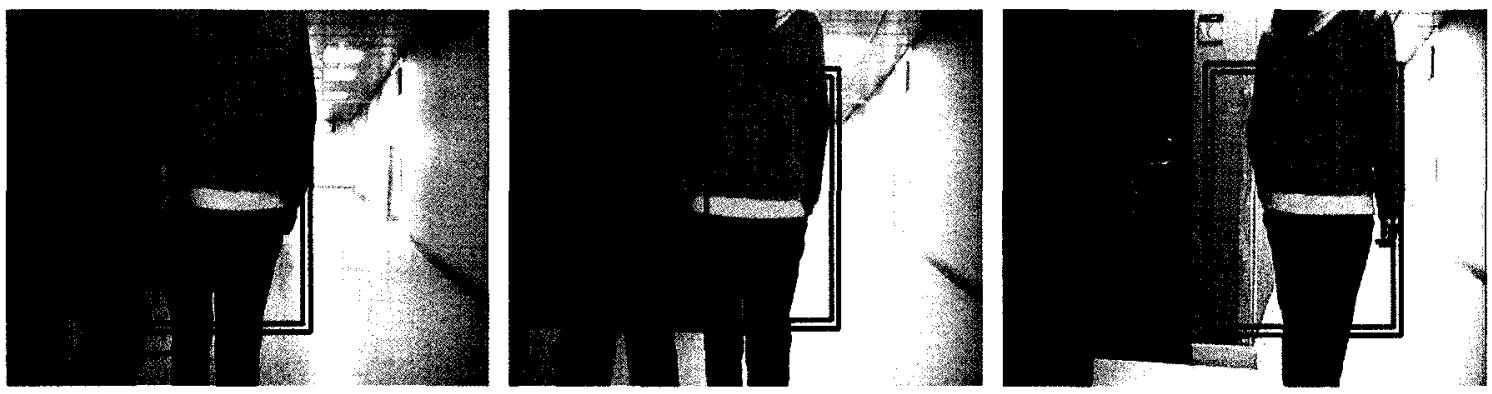

(c-1)

(c-2)

(c-3)

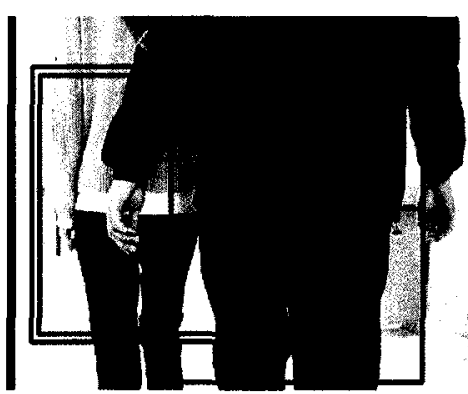

(c-4)

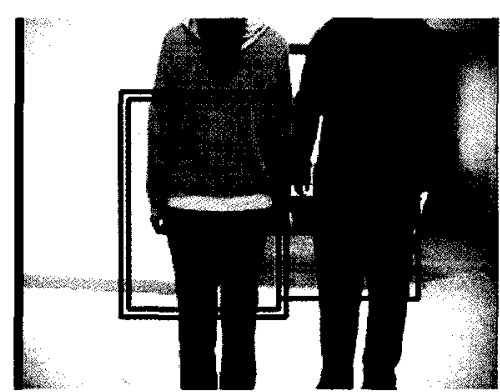

(c-5)

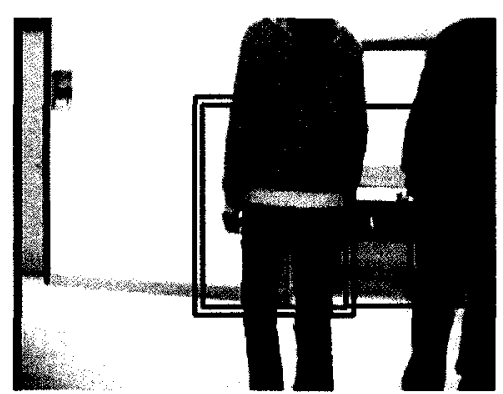

(c-6)

(c) Frames recorded by the onboard computer 


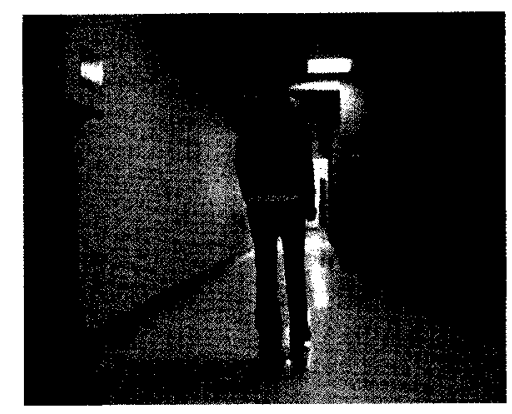

(d-1) Frame 86

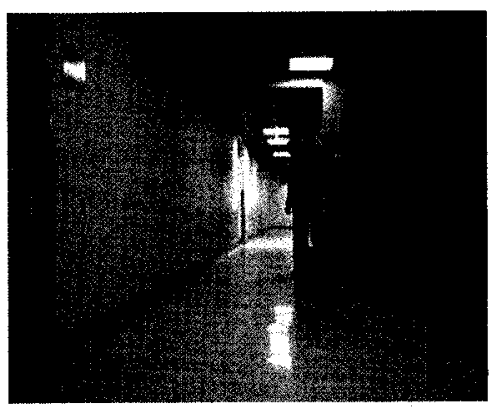

(d-4) Frame 330

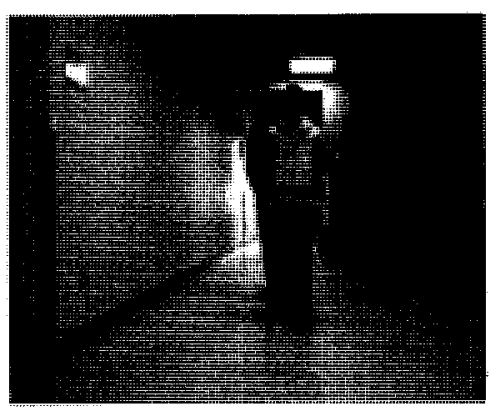

(d-7) Frame 496

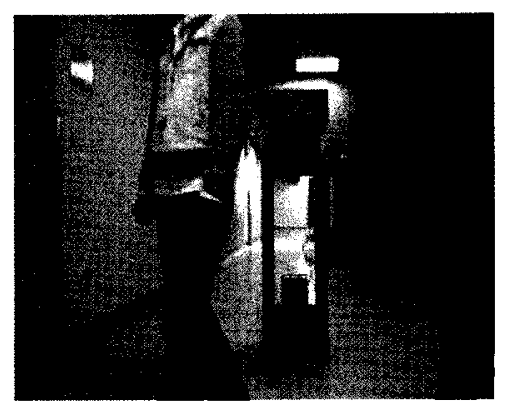

(d-10)Frame 616

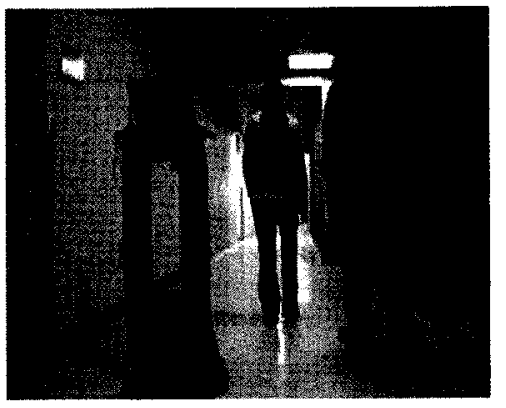

(d-2) Frame 125

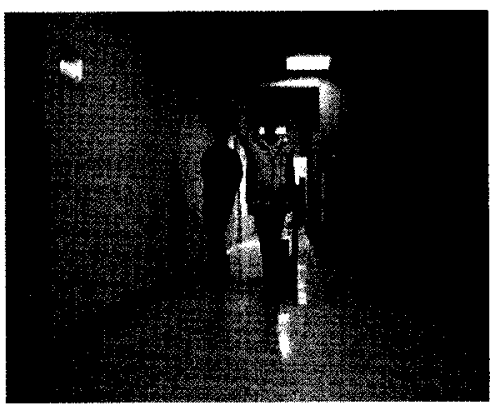

(d-5) Frame 440

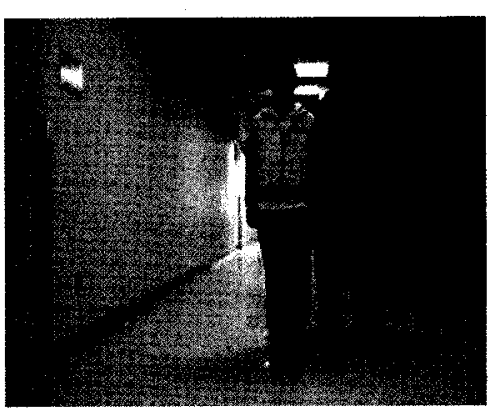

(d-8) Frame 536

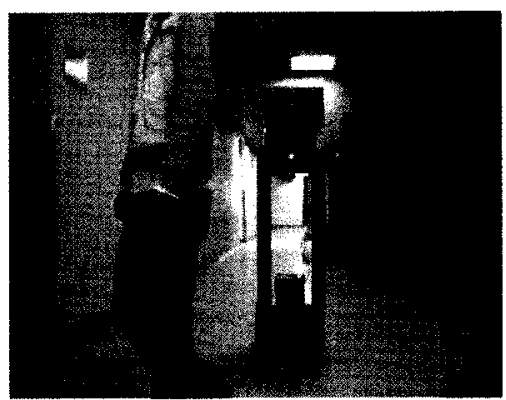

(d-11) Frame 620

(d) Video frames

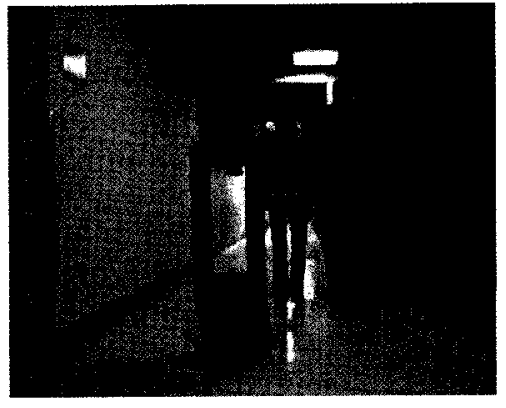

(d-3) Frame 156

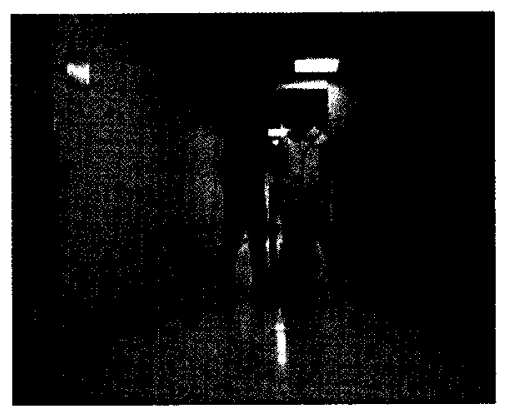

(d-6) Frame 472

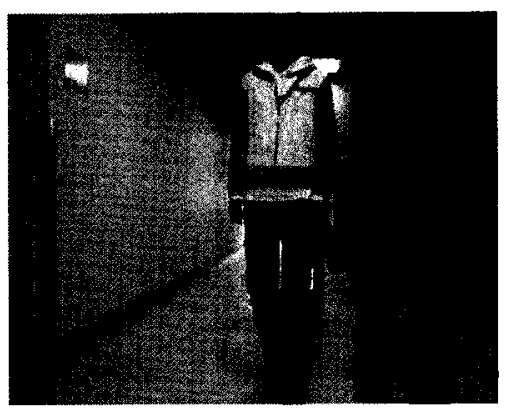

(d-9) Frame 580

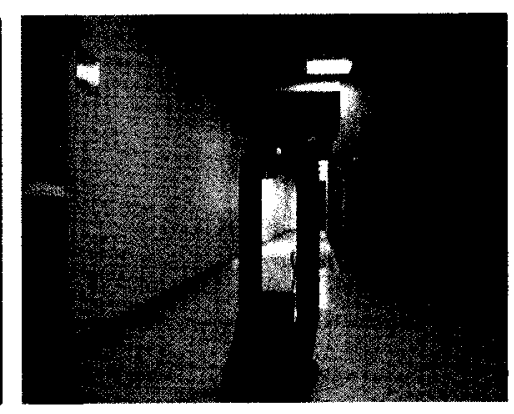

(d-12) Frame 645

Figure 5.5. Target person recognition: person 1 
From Figure 5.5 (d-1) to Figure 5.5 (d-3), the target person was walking into the corridor followed by the robot and the non-target person walked into the field of view of the robot. In Figure 5.5 (d-4) to Figure 5.5 (d-6), the target and non-target persons turned to opposite directions. Figure 5.5 (d-7) to Figure 5.5 (d-9) present that the non-target person crossed the robot and the target person and then walked along with the target. Figure $5.5(\mathrm{~d}-10)$ to Figure $5.5(\mathrm{~d}-12)$ show that the target and non-target persons turned to opposite directions and the robot kept following the target.

\subsubsection{The Experiment on Person 2}

Figure 5.6 gives the results that the target person is switched to person 2. The trajectories of the target and non-target person are the same as Figure 5.5 (a).

Figure 5.6 (a) is the segmented target person. Figure 5.6 (b) are the frames recorded by the onboard computer. Observe Figure 5.6 (b-3), the laser range finder mistook the corner of the door as a human candidate, which was recognized as the nontarget person by the image verification. Figure 5.6 (c) are the video frames. 


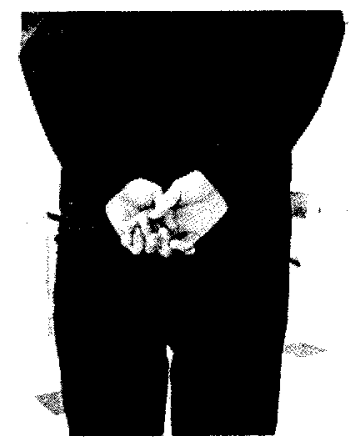

(a) Segmented target image
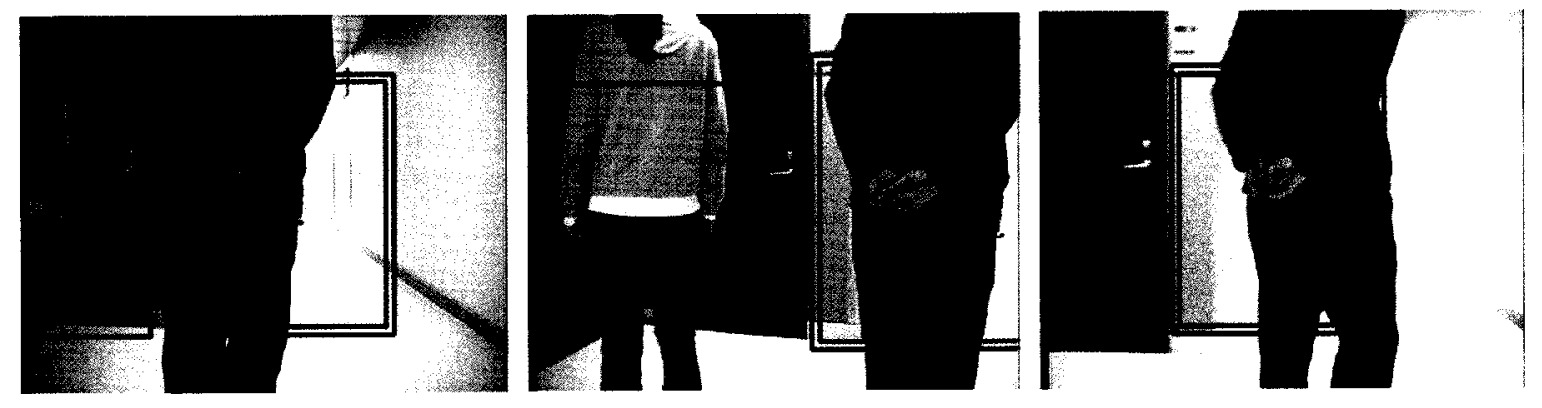

$(b-1)$

(b-2)

$(b-3)$
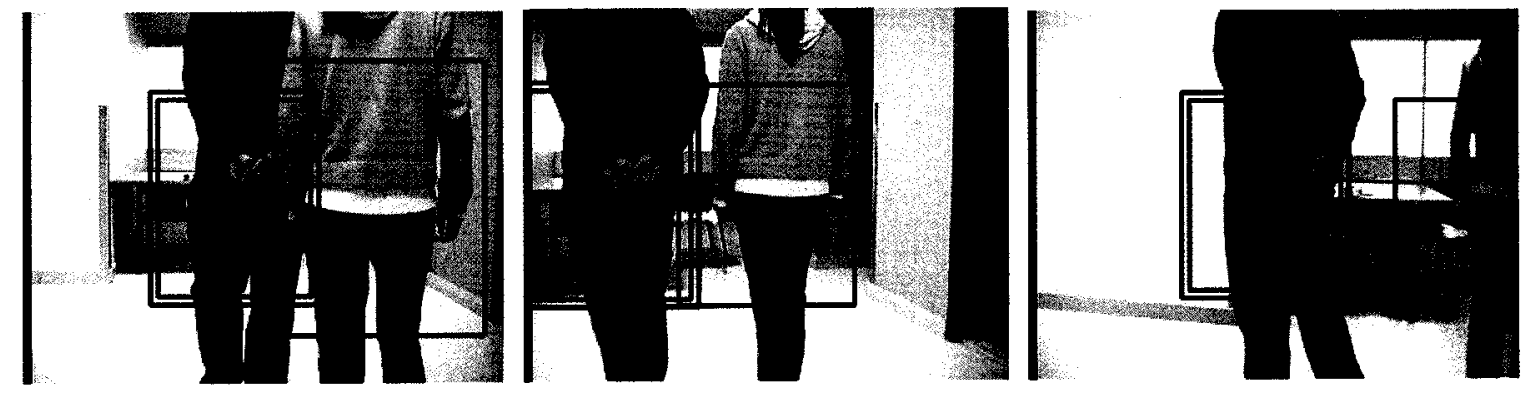

(b-4)

(b-5)

(b-6)

(b) Frames recorded by the onboard computer 


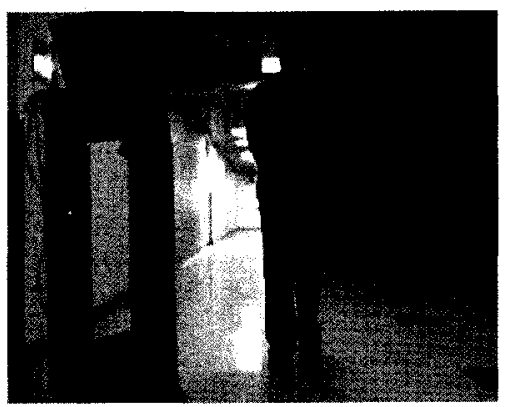

(c-1) Frame 56

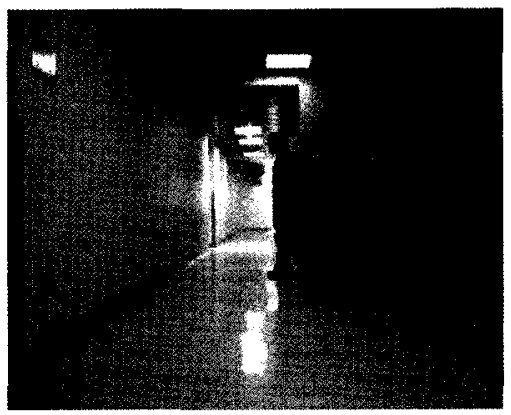

(c-4) Frame 278

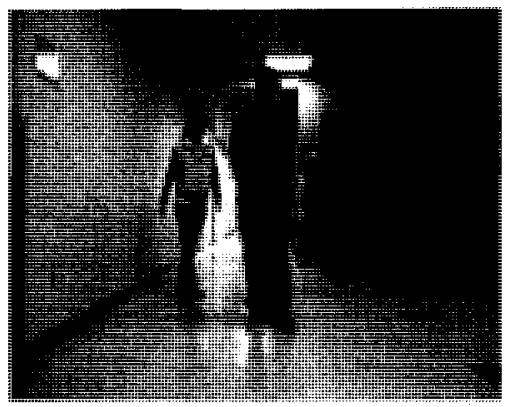

(c-7) Frame 528

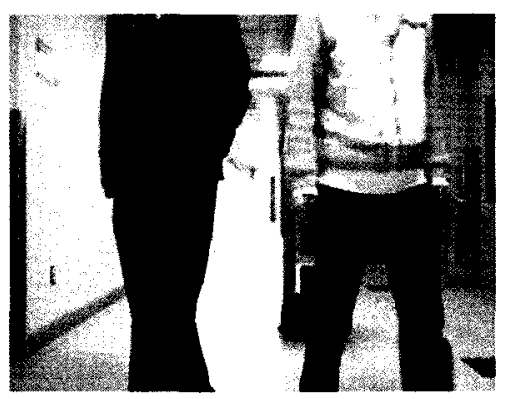

(c-10) Frame 648

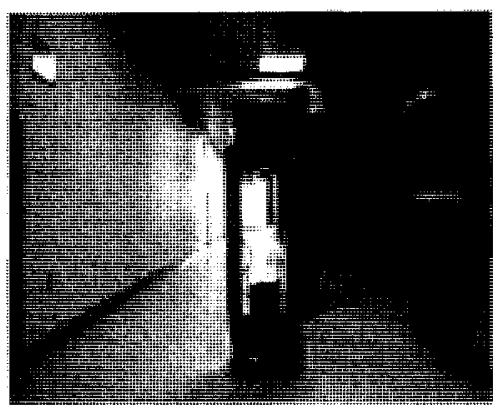

(c-2) Frame 125

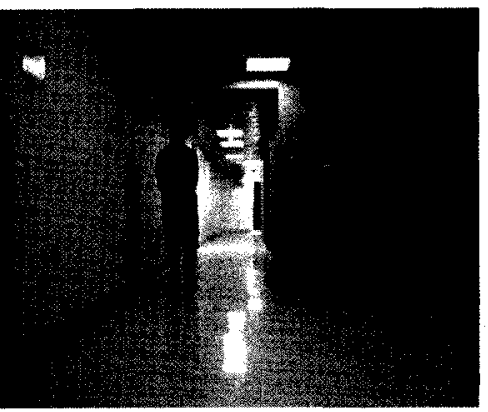

(c-5) Frame 398

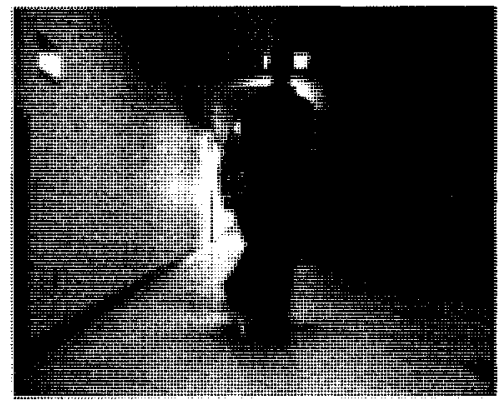

(c-8) Frame 548

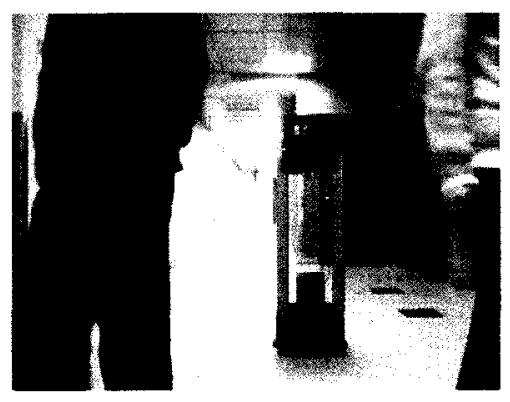

(c-11) Frame 661

(c) Video frames

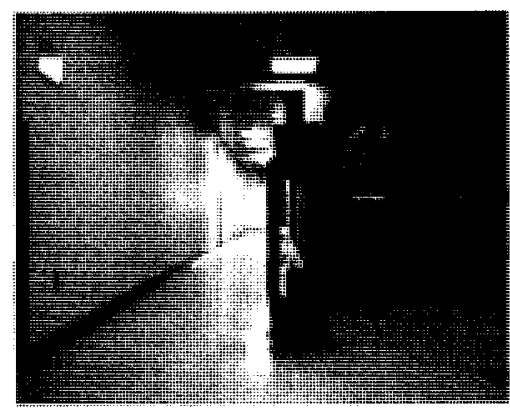

(c-3) Frame 156

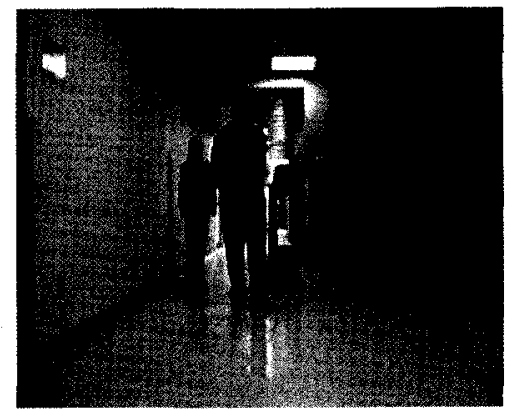

(c-6) Frame 482

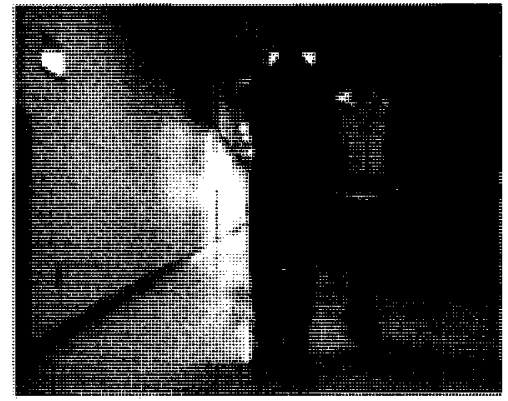

(c-9) Frame 592

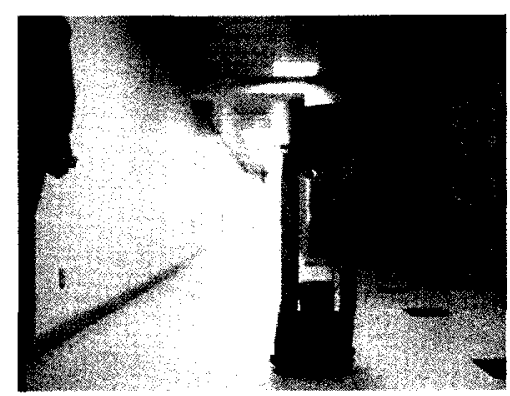

(c-12) Frame 680

Figure 5.6. Target person recognition: person 2 


\subsubsection{Maximum Angular Velocity}

The maximum pan speed of the camera is $90^{\circ} / \mathrm{s}$, and the maximum rotation angle

of the robot is set to be $100^{\circ} / \mathrm{s}$. If there is no translation command sent to the robot, the maximum angular velocity of the person could be $190^{\circ} / \mathrm{s}$.

However, the robot has difficulty to turn when it is moving straight at the maximum speed $(750 \mathrm{~mm} / \mathrm{s})$. Due to this fact, the experimental maximum angular velocity of the person is $40^{\circ} / \mathrm{s}$.

\subsubsection{Tracking Range}

When the person is walking away from the robot, the number of laser readings on one human leg will be less than 5 , which will lead the leg detection to be failure. According to experimental data in Table 4.1, the maximum distance should be less than $2.5 \mathrm{~m}$ whereas the maximum distance varies depending on different experimental targets due to different leg diameters.

On the other hand, when the person is turning, the robot will react to the turning and may hit the corners or other objects. Therefore the person is expected to be close to the robot when changing his or her direction.

Combining the two aspects above, it is recommended that the tracked person keeps being within the circle that is centered at the robot with radius at around $2 \pm 0.5 \mathrm{~m}$ in a spacious environment. 


\section{Chapter 6 Conclusions and Future Work}

This chapter gives the conclusions of this thesis and recommends some possible future work.

\subsection{Conclusions}

In this thesis, a robust and real-time human tracking mobile robot system is developed. Some conclusions are listed below.

1. The target person model constructed in the pre-tracking stage performs a predominant role for this moving person tracking application. The non-target objects are discarded with the help of the target model to accomplish the real-time tracking. The target model contains the location information from the laser range finder and the image information from the camera. This model construction method can be applied to model the moving objects that are "seen" by the robot (both the camera and the laser range finder) in other applications, such as robot soccer games. The problem for this model is that if the image information (such as the colour or edge orientation distribution) changes drastically during the tracking, an online model updating is needed.

2. The leg detection algorithm developed in this thesis uses the leg width, the leg shape and the leg's possible location. The comparison between the leg detection algorithm developed in this thesis with state-of-the-art leg detection algorithm shows that it has low undetected probability. Since the consideration of the human leg's motion (leg detection criteria 3 in section 3.4.2), the leg-like objects 
(detected by the leg detection criteria 1 and 2) that are far away from the leg positions in the previous laser frame will not be recognized as leg candidates, therefore the false alarm probability is also be lowered. This leg detection algorithm can be used for human detection with satisfactory detection probability even without the camera verification.

3. By fusing the information from the camera and the laser range finder, the robust tracking is fulfilled. With higher computation power, better tracking performance can be expected by further fusing the information from other types of sensors or devices

\subsection{Future Work}

1. The current research is focusing on the human tracking application. When following a person, the robot will take a shortcut path, which means the robot may turn as soon as it detects the turning of the target (as mentioned in Chapter 4, the robot will turn if the angle between the target and the robot is larger than $+6^{\circ}$ or less than $-6^{\circ}$ ). Thus the robot may be prone to hit objects on its way. One possible solution to this problem is to store a map of the environment in the robot in advance. During the course of tracking, the robot will be able to locate itself in the map. Then at each time step, the robot can plan its path based on the trajectory of the person and the environment around itself. Another possible way for solving the problem is to add an obstacle avoidance function, which of course will make the problem more complicated.

2. The camera and laser data fusion is based on a simple coordinate transformation (transform the human position in the laser frame to the image frame). The 
rectangle image region, which represents the person in the image frame, is determined by the location information of the person in the laser frame and the information from the target model. In the experiments, the camera and the laser range finder are not calibrated, which means the size and the center of the rectangle in the image frame may contain some errors. A more accurate person localization in the image frame can be obtained by calibrating the laser range finder and the camera in advance.

3. Simultaneous human tracking and environmental map building will be an attractive topic. Techniques of data fusion for environmental map building can be explored. 


\section{References}

[1] E. Menegatti, A. Pretto, A. Scarpa, and E. Pagello, "Omnidirectional Vision Scan Matching for Robot Localization in Dynamic Environments", IEEE Trans. on Robotics, Vol. 22, Issue 3, June 2006, pp. 523-535.

[2] B. Krose, R. Bunschoten, S. T. Hagen, B. Terwijn, and N. Vlassis, "Household Robots Look and Learn: Environment Modeling and Localization from an Omnidirectional Vision System", IEEE Magazine on Robotics \& Automation, Vol. 11, Issue 4, Dec. 2004, pp. 45-52.

[3] J. A. Castellanos, J. Neira, and J. D. Tardos, "Multisensor Fusion for Simultaneous Localization and Map Building", IEEE Trans. on Robotics and Automation, Vol. 17, Issue 6, Dec. 2001, pp. 908-914.

[4] C. Wang, and C. Thorpe, "Simultaneous Localization and Mapping with Detection and Tracking of Moving Objects", Proc. of IEEE, Int. Conference on Robotics and Automation, Vol. 3, May 2002, pp. 2918-2924.

[5] A. J. Davison, I. D. Reid, N. D. Molton, and O. Stasse, "MonoSLAM: Real-Time Single Camera SLAM", IEEE Trans. On Pattern Analysis and Machine Intelligence, Vol. 29, Issue 6, June 2007, pp. 1052-1067.

[6] G. N. Desouza, and A. C. Kak, "Vision for Mobile Robot Navigation: A Survey", IEEE Trans. on Pattern Analysis and Machine Intelligence, Vol. 24, Issue 2, Feb. 2002, pp. 237-267.

[7] A. S. Sekmen, M. Wilkes, and K. Kawamura, "An Application of Passive HumanRobot Interaction: Human Tracking Based on Attention Distraction", IEEE Trans. on Systems, Man and Cybernetics, Part A, Vol. 32, Issue 2, March 2002, pp. 248259. 
[8] T. P. Spexard, M. Hanheide, and G. Sagerer, "Human-Oriented Interaction with an Anthropomorphic Robot”, IEEE Trans. on Robotics, Vol. 23, Issue 5, Oct, 2007, pp. 852-862.

[9] R. Stiefelhagen, H. K. Ekenel, C. Fugen, P. Gieselmann, H. Holzapfel, F. Kraft, K. Nickel, M. Voit, and A. Waibel, "Enabling Multimodal Human-Robot Interaction for the Karlsruhe Humanoid Robot", IEEE Trans. on Robotics, Vol. 23, Issue 5, Oct. 2007, pp. 840-851.

[10] B. Jensen, N. Tomatis, L. Mayor, A. Drygajlo, and R. Siegwart, "Robots Meet Humans-Interaction in Public Spaces", IEEE Trans. on Industrial Electronics, Vol. 52, Issue 6, Dec. 2005, pp. 1530-1546.

[11] X. Xie, and K. Lam, "Gabor-based Kernel PCA with Doubly Nonlinear Mapping for Face Recognition with A Single Face Image", IEEE Trans. on Image Processing, Vol. 15, Issue 9, Sept. 2006, pp. 2481-2492.

[12] P. Vadakkepat, P. Lim, L. C. De Silva, L. Jing, and L. L. Ling, "Multimodal Approach to Human-Face Detection and Tracking", IEEE Trans. on Industrial Electronics, Vol. 55, Issue 3, March 2008, pp. 1385-1393.

[13] C. J. Yang, R. Duraiswami, and L. Davis, "Fast Multiple Object Tracking via A Hierarchical Particle Filter", Proc. of IEEE, Int. Conference on Computer Vision, Vol. 1, Oct. 2005, pp. 212 - 219.

[14] A. Agarwal, and B. Triggs, "Recovering 3D Human Pose from Monocular Images", IEEE Trans. on Pattern Analysis and Machine Intelligence, Vol. 28, Issue 1, Jan. 2006, pp. 44-58.

[15] P. Chakravarty, and R. Jarvis "Panoramic Vision and Laser Range Finder Fusion for Multiple Person Tracking", Proc. of IEEE, Int. Conference on Intelligent Robots and Systems, Oct. 2005, pp. 2949 - 2954. 
[16] J. H. Lee, Y. S. Kim, B. K. Kim, K. Ohba, H. Kawata, A. Ohya, and S. Yuta, "Security Door System Using Human Tracking Method with Laser Range Finders", Proc. of IEEE, Int. Conference on Mechatronics and Automation, Aug. 2007, pp. 2060-2065.

[17] E. Grosso, and M. Tistarelli, "Active/Dynamic Stereo Vision", IEEE Trans. on Pattern Analysis and Machine Intelligence, Vol. 17, Issue 9, Sept. 1995, pp. 868879.

[18] P. Saeedi, P. D. Lawrence, and D. G. Lowe, "Vision-Based 3-D Trajectory Tracking for Unknown Environments", IEEE Trans. on Robotics, Vol. 22, Issue 1, Feb. 2006, pp. 119-136.

[19] Z. Zhu, D. R. Karuppiah, E. M. Riseman, and A. R. Hanson, "Keeping Smart, Omnidirectional Eyes on You", IEEE Magazine on Robotics \& Automation, Vol. 11, Issue 4, Dec. 2004, pp. 69-78.

[20] D. S. Lee, "Effective Gaussian Mixture Learning for Video Background Subtraction", IEEE Trans. on Pattern Analysis and Machine Intelligence, Vol. 27, Issue 5, May 2005, pp 827-832.

[21] R. Cucchiara, C. Grana, M. Piccardi, and A. Prati, "Detecting Moving Objects, Ghosts, and Shadows in Video Streams", IEEE Trans. on Pattern Analysis and Machine Intelligence, Vol. 25, Issue 10, Oct. 2003, pp. 1337-1342.

[22] A. Verri, and T. Poggio, "Motion Field and Optical flow: Qualitative Properties", IEEE Trans. On Pattern Analysis and Machine Intelligence, Vol. 11, Issue 5, May 1989 , pp. 490-498.

[23] A. G. Bors, and L. Pitas, "Prediction and Tracking of Moving Objects in Image Sequences", IEEE Trans on Image Processing, Vol. 9, Issue 8, Aug. 2000, pp. 1441-1445. 
[24] P. Stepan, M. Kulich, and L. Preucil, "Robust Data Fusion with Occupancy Grid", IEEE Trans. on Systems, Man, and Cybernetics, Part C: Applications and Reviews, Vol. 35, Issue 1, Feb. 2005, pp. 106-115.

[25] J. H. Lee, T. Tsubouchi, K. Yammamoto, and S.Egawa, "People Tracking Using a Robot in Motion with Laser Range Finder", Proc. of IEEE, Int. Conference on Intelligent Robotics and Systems, Oct. 2006, pp. 2936 - 2942.

[26] X. Xu, and B. Li, "Adaptive Rao-Blackwellized Particle Filter and Its Evaluation for Tracking in Surveillance", IEEE Trans. on Image Processing, Vol. 16, Issue 3, March 2007, pp. 838-849.

[27] K. O. Arras, O. M. Mozos, and W. Burgard, "Using Boosted Features for the Detection of People in 2D Range Data", Proc. of IEEE, Int. Conference on Robotics and Automation, April 2007, pp. 3402-3407.

[28] E. A. Topp, and H. I. Christensen, "Tracking for Following and Passing Persons", Proc. of IEEE, Int. Conference on Intelligent Robots and Systems, Aug. 2005, pp. 2321-2327.

[29] J. Xavier, M. Pacheco, D. Castro, and A. Ruano, "Fast Line, Arc/Circle and Leg Detection from Laser Scan Data in a Player Driver", Proc. of IEEE, Int. Conference on Robotics and Automation, April 2005, pp. 3930-3935.

[30] G. L. Foresti, and C. S. Regazzoni, "Multisensor Data Fusion for Autonomous Vehicle Navigation in Risky Environments", IEEE Trans. On Vehicular Technology, Vol. 51, Issue 5, Sept. 2002, pp. 1165-1185.

[31] D. M. Gavrila, "Sensor-Based Pedestrian Protection", IEEE Magazine on Intelligent Systems, Vol. 16, Issue 6, Nov-Dec, 2001, pp. 77-81.

[32] K. Morioka, J. H. Lee, and H. Hashimoto, "Human-Following Mobile Robot in a Distributed Intelligent Sensor Network", IEEE Trans. on Industrial Electronics, Vol. 51, Issue 1, Feb. 2004, pp. 229- 237. 
[33] A. Feldman, S. Adams, M. Hybinette, and T. Balch, "A Tracker for Multiple Dynamic Targets Using Multiple Sensors", Proc. of IEEE, Int. Conference on Robotics and Automation, April 2007, pp.3140 - 3141.

[34] J. S. Cui, H. B. Zha, H. J. Zhao, and R. Shibasaki, "Robust Tracking of Multiple People in Crowds Using Laser Range Scanners", Proc. of IEEE, Int. Conference on Pattern Recognition, Vol. 4, 2006, pp. 857-860.

[35] A. Fod, A. Howard, and M. J. Mataric, "A Laser-Based People Tracker", Proc. of IEEE, Int. Conference on Robotics and Automation, Vol. 3, May 2002, pp. 30243029 .

[36] P. R. Liu, M. Q. H. Meng, and P. X. Liu, "Moving Object Segmentation and Detection for Monocular Robot Based on Active Contour Model", IEEE Electronics Letters, Vol. 41, Issue 24, Nov. 2005, pp.1320-1322.

[37] S. K. Goldenstein, "A Gentle Introduction to Predictive Filters", Revista de Informatica Teorica e Aplicada (RITA), Vol. XI, Number 1, Oct. 2004, pp. 61-89.

[38] M. S. Arulampalam, S. Maskell, N. Gordon, and T. Clapp, "A Tutorial on Particle Filters for Online Nonlinear/Non-Gaussian Bayesian Tracking", IEEE Trans. on Signal Processing, Vol. 50, Issue 2, Feb. 2002, pp. 174-187.

[39] X. Yun, and E. R. Bachmann, "Design, Implementation, and Experimental Results of a Quaternion-Based Kalman Filter for Human Body Motion Tracking", IEEE Trans on Robotics, Vol. 22, Issue 6, Dec. 2006, pp. 1216-1227.

[40] M. Bertozzi, A. Broggi, A. Fascioli, A. Tibaldi, R. Chapuis, and F.chausses, "Pedestrian Localization and Tracking System with Kalman Filtering", IEEE, International Vehicles Symposium, June 2004, pp. 584-589.

[41] R. Gockley, J. Forlizzi, and R. Simmons, "Natural Human tracking Behavior for Social Robots", Proc. of ACMIEEE, Int. Conference on Human-robot interaction, March 2007, pp. 17-24. 
[42] C. Chang, and R. Ansari, "Kernel Particle Filter for Visual Tracking", IEEE Signal Processing Letters, Vol. 12, Issue 3, March 2005, pp. 242-245.

[43] S. K. Zhou, R. Chellappa, and B. Moghaddam, "Visual Tracking and Recognition Using Appearance-adaptive Models in Particle Filters", IEEE Trans. on Image Processing, Vol. 13, Issue 11, Nov. 2004, pp. 1491-1506.

[44] B. Kwolek, "Person Following and Mobile Camera Localization Using Particle Filters", Int. workshop on Robot Motion and Control, June 2004, pp.265-270.

[45] W. Zajdel, Z. Zivkovic, and B. J. A. Krose, "Keeping Tracking of Humans: Have I Seen This Person Before", Proc. of IEEE, Int. Conference on Robotics and Automation, Vol. 18-21, April 2005, pp. 2081-2086.

[46] N. Bellotto, and H. Hu, "Vision and Laser Data Fusion for Tracking people with a Mobile Robot", Proc. of IEEE, Int. Conference on Robotics and Biomimetics, Dec. 2006, pp.7-12.

[47] P. Vadakkepat, and L. Jing, "Improved Particle Filter in Sensor Fusion for Tracking Randomly Moving Object”, IEEE Trans. on Instrumentation and Measurement, Vol. 55, Issue. 5, Oct. 2006, pp. 1823-1832.

[48] D. Schulz, W. Burgard, D. Fox, and A. B. Cremers, "Tracking Multiple Moving Targets with a Mobile Robot using Particle Filters and Statistical Data Association", Proc. of IEEE, Int. Conference on Robotics and Automation, Vol. 2, May 2001, pp. 1665-1670.

[49] E. Maggio, F. Smerladi, and A. Cavallaro, "Adaptive Multifeature Tracking in a Particle Filtering Framework", IEEE Trans. on Circuits and Systems for Video Technology, Vol. 17, Issue. 10, Oct. 2007, pp. 1348-1359.

[50] P. Perez, C. Hue, J. Vermaak, and M. Gangnet, "Colour-Based Probabilistic Tracking", Proceedings of the $7^{\text {th }}$ European Conference on Computer Vision Part I, May-June, 2002, pp. 661-675. 
[51] W. T. Freeman, and M. Roth, "Orientation Histograms for Hand Gesture Recognition", International workshop on Automatic Face and Gesture Recognition, June 1995, pp. 296-301.

[52] D. Comaniciu, V. Ramesh, and P. Meer, "Kernel-Based Object Tracking", IEEE Trans. on Pattern Analysis and Machine Intelligence, Vol. 25, Issue 5, May 2003, pp. 564-577.

[53] A. Doucet, S. Godsill, and C. Andrieu, "On sequential Monte Carlo Sampling Methods for Bayesian Filtering", Statistics and Computing, Vol. 10, 2000, pp. 197208.

[54] D. A. Forsyth, and J. Ponce, Computer Vision: A Modern Approach, Prentice Hall, 2002.

[55] Intel, Open Source Computer Vision C++ Library, Retrieved Aug. 18, 2007, from website: http://opencvlibrary.sourceforge.net

[56] ActivMedia Robotics, ActivMedia Robotics Interface for Application (ARIA) C++ library, Retrieved Aug. 18, 2007, from website: http://mobilerobots.com

[57] ActivMedia, Performance PeopleBot Operation Manual, Version 5, Aug. 2004.

[58] ActivMedia Robotics, PTZ Robotic Cameras, 2004.

[59] SICK Sensor Intelligence, LMS200/211/221/291 Laser Measurement Systems, 2006, Retrieved Oct. 5, 2007, from website: www.mysick.com/saqqara/.

[60] M. Pfeiffer, "A brief Introduction to Particle Filters", Retrieved July 6, 2007, from website: http://www.igi.tugraz.at/pfeiffer/documents/particlefilters.pdf 